\title{
ANÁLISE DE DEPENDÊNCIA POR TRAJETÓRIAS
}

\author{
DORIS ALBINA GÓMEZ TICERÁN \\ Licenciada em Estatística
}

Orientador: Prof. Dr. JOSÉ ANTÔNIO CORDEIRO

Tese apresentada à Escola Superior de Agricultura "Luiz de Queiroz", da Universidade de São Paulo, para obtenção do título de Doutor em Agronomia, Área de Concentração: Estatística e Experimentação Agronômica.

P I R A C I C A B A

Estado de São Paulo - Brasil

Novembro - 1996 
Dados Internacionais de catalogação na Publicação (CIP) DIVISĀO DE BIBLIOTECA E DOCUMENTAÇĀO - Campus "Luiz de QueirOz"/USP

\section{Gómez Ticerán, Doris Albina}

Análise de dependência por trajetórias / Doris Albina Gómez Ticerán. . . Piracicaba, 1996.

106p. : il.

Tese (doutorado) - - Escola Superior de Agricultura Luiz de Queiroz, 1997. Bibliografia

1. Análise multivariada 2. Dados categóricos 3. Estatística aplicada I. Titulo 


\section{ANÁLISE DE DEPÊNDENCIA POR TRAJETÓRIAS}

\section{DORIS GÓMEZ TICERÁN}

Aprovado em: 19/02/1997

Comissão julgadora:

Prof. Dr. José Antônio Cordeiro

IBILCE/UNESP

Prof. Dr. Antônio Francisco Iemma

Prof $^{a .} \operatorname{Dr}^{a}$. Clarice Garcia Borges Demétrio

Prof. Dr. Luiz Carlos Baida

Prof. Dr. Belmer Garcia Negrillo

$$
\begin{array}{r}
\text { ESALQ/USP } \\
\text { ESALQ/USP } \\
\text { IBILCE/UNESP } \\
\text { IMECC/UNICAMP }
\end{array}
$$

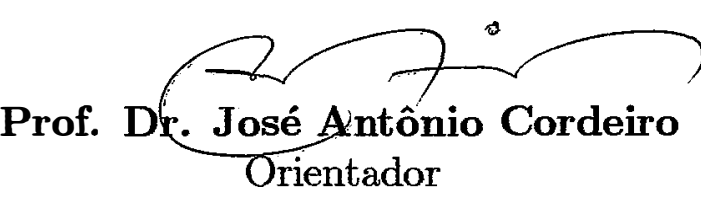




\section{DEDICAÇÃO}

A Yakov,
sentido do que faço,

dedico com carinho e amor. 
A Mario, companheiro de todos os momentos

e exemplo de união, dedicação e amor.

Ao meu pai Luis que me deu exemplo de caráter, amor e justiça.

À minha mãe Guillermina pelo seu desvelo e incentivo, em todas as fases da minha vida.

Aos quais da mesma forma, dedico este trabalho. 


\section{AGRADECIMENTOS}

Ao Professor Dr. José Antônio Cordeiro, orientador desta tese, pelo apoio, ensinamentos valiosos e pelo exemplo de figura humana digna. Mesmo não pertencendo ao quadro de docentes da ESALQ aceitou ser o meu orientador, fato que nunca esquecerei.

À professora Dra. Clarice Demétrio por ter sido minha orientadora na primeira fase dos meus estudos de doutorado e pelas sugestôes para o aperfeiçoamento deste trabalho.

Aos Professores do Departamento de Matemática e Estatística da ESALQ/USP, pelos ensinamentos e consideração.

Aos professores da Faculdade de Ciências Matemáticas da U.N.M. de San Marcos de Lima Perú, que me apoiaram na iniciação dos meus estudos de Doutorado.

Aos funcionários do Departamento de Matemática e Estatística, pela atenciosa cooperação ao longo de todo o curso.

À Sra. Kátia de Andrade Ferraz, bibliotecária da Biblioteca Central da ESALQ/USP, pela revisão das referências bibliográficas. aperfeiçoamento deste trabalho.

À minha amiga Imaculada Montebelo e a toda sua familia por terenme aberto o seu coração, o que ajudou na minha adaptação e da minha família aqui no Brasil. Também pelo precioso apoio na datilografia, na correção do Português e no aperfeiçoamento dos gráficos.

À minha amiga Roseli pelos aportes e sugestões no uso do proces- 
sador de texto (LATEX) para digitar este trabalho.

Ao meu filho, Yakov, pelo auxilio na elaboração dos gráficos deste trabalho.

Aos Colegas de curso, Claudia, Gil , Imaculada, Liciana, Luis, Pilar e Roseli, pelo companherismo e constante intercâmbio de idéias.

À minha família, Mario e Yakov, pela compreensão e a força recebida, e sem as quais seria impossível a conclusão dos meus estudos.

Aos meus pais Luis e Guillermina que apesar da distância sempre me alentaram na continuação dos meus estudos.

Ao Dr. M. Bélo por ter cedido os dados experimentais usados neste trabalho.

À ESALQ/USP pela oportunidade de estudo.

À CAPES que me concedeu uma bolsa de estudo do Convênio PERÚ-PEC/PG, tornando possível minha permanência no Brasil, para o desenvolvimento deste trabalho.

A todos aqueles que, direta ou indiretamente, contribuiram para a conclusão deste trabalho. 


\section{SUMÁRIO}

\section{INTRODUÇÃO}

2 REVISÃO BIBLIOGRÁFICA $\quad 10$

2.1 Análise de dependência e co-dependência populacional $\ldots 10$

2.2 Análise de trajetória numérica . . . . . . . . . . . . 18

2.2 .1 Considerações gerais . . . . . . . . . . . 18

2.2.2 Análise de trajetória em modelos de regressão múltipla $\ldots$. . 28

\section{DESENVOLVIMENTO TEÓRICO DA}

TRANSPOSIÇÃO DA METODOLOGIA DE ANÁLISE DE TRAJETÓRIA PARA DADOS CATEGÓRICOS 37

3.1 Introdução . . . . . . . . . . . . . . . . . . 37

3.2 Desenvolvimento do modelo . . . . . . . . . . . . 40

3.2 .1 Considerações gerais . . . . . . . . . . . . . . 40

3.2.2 Análise de trajetória através de um modelo de "regressão" . . 44

3.2.3 Todos os níveis da categoria são níveis respostas . . . . . . . . 49

3.3 Estimação . . . . . . . . . . . . . . . . . . 55

3.3 .1 Considerações gerais . . . . . . . . . . . 55

3.3.2 Teorema da análise por caminhos em tabelas de contingência de dupla entrada . . . . . . . . . . . . 57

3.4 Testes de hipótese para os coeficientes de trajetória . . . . . . 60

4 APLICAÇÃO DA METODOLOGIA 62

4.1 Descrição dos dados . . . . . . . . . . . . . . . . . . 62

4.2 Resultados da análise de dependência . . . . . . . . . . 6 65

4.3 Resultados da análise de trajetórias . . . . . . . . . . . . . . 67

4.3.1 Com dados não padronizados . . . . . . . . . . . 67 
4.3.2 Com dados padronizados $\ldots \ldots \ldots \ldots . \ldots 72$

4.3.3 Considerando apenas relações de associação . . . . . . . . 73

4.4 Decomposição dos coeficientes de co-dependência . . . . . . . . 80

4.5 Interterpretações dos coeficientes de trajetórias . . . . . . . . . . 83

4.6 Testes de hipóteses . . . . . . . . . . . . . . 86

5 CONCLUSÕES 101

6 REFERÊNCIAS BIBLIOGRÁFICAS 103

\section{LISTA DE FIGURAS}

1 Uma relação causal com um antecedente . . . . . . . . . . . . 24

2 Uma relação causal com dois antecedentes não correlacionados . . . 24

3 Gráfico de caminhos com duas variáveis exógenas correlacionadas . . 25

4 Diagrama de trajetória para o modelo de regressão . . . . . . . . . 29

5 Gráfico de Trajetória para o modelo proposto . . . . . . . . . 4 45

6 Gráfico de Trajetória proposto para a espécie Drosophyla . . . . . . 68

$7 \quad$ Gráfico de trajetória para as espécies Drosophila . . . . . . . . . 84

8 Gráfico de trajetórias final para as espécies Drosophyla . . . . . . . 93

9 Gráfico de trajetórias para Saúde Mental . . . . . . . . . . . . . 97

\section{LISTA DE TABELAS}

1 Exemplo hipotético sobre a influência psico-social na incidência de câncer em mulheres . . . . . . . . . . . . . . . . . 2

2 Dados individuais de nascimentos ocorridos no Município de São Paulo, $1978 \ldots \ldots \ldots \ldots \ldots \ldots \ldots$

3 Classificação de 20878 nascimentos ocorridos no Município de São Paulo, $1978 \ldots \ldots \ldots \ldots \ldots \ldots \ldots$. . . . . . . . . 4 
4 Classificação de pessoas com câncer de pele segundo o tipo de câncer e o local da lesão . . . . . . . . . . . . . . . . 6

5 Classificação de pacientes segundo o tipo de vacina e níveis de anticorpo hemoglutina encontrado no sangue . . . . . . . . . . . 6

6 Classificação de 1660 pessoas segundo a saúde mental e o estado sócio-

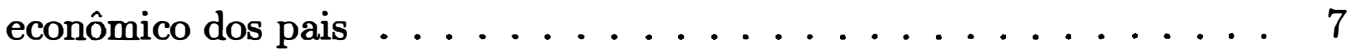

7 Classificação das moscas segundo leveduras . . . . . . . . . . . . 63

8 Dependências e Co-dependências da Espécie Drosophyla . . . . . . . 66

9 Coeficientes de Co-dependências entre os níveis da Espécie Drosophyla 66

10 Decomposição dos Coeficientes de Co-dependência . . . . . . . . . . . 85

11 Resultados dos Testes de Hipóteses para a equação ( 81) . . . . . . 88

12 Resultados dos Testes de Hipóteses para a equação (82) . . . . . . . 89

13 Resultados dos Testes de Hipóteses para a equação (83) . . . . . 90

14 Resultados dos Testes de Hipóteses para a equação (84) . . . . . . 91

15 Resultados dos Testes de Hipóteses para a equação ( 85) . . . . . . 92

16 Decomposição Final dos Coeficientes de Co-dependência . . . . . . . 95

17 Coeficientes de Co-dependência entre os níveis de Saúde Mental . . 96

18 Dependências e Co-dependências entre os níveis de saúde mental . . . 96

19 Decomposição dos Coeficientes de Co-dependência . . . . . . . . . . 98

20 Resultados dos Testes de Hipóteses para os dados de Saúde mental . 99 


\title{
ANÁLISE DE DEPÊNDENCIA POR TRAJETÓRIAS
}

\author{
Autor: Doris Albina Gómez Ticerán \\ Orientador: Prof. Dr. José Antônio Cordeiro
}

\section{RESUMO}

Uma proposta de Análise de Trajetória com variáveis categóricas é apresentada com base nos coeficientes de co-dependência, segundo a metodologia encontrada em Cordeiro(1990).

Assim, em uma tabela onde numa das dimensões estão as espécies de algum animal e na outra os ambientes, aplicando-se as técnicas convencionais pode-se testar se há ou não evidência de associação entre as duas categorias. Em havendo, ajusta-se algum modelo explicativo da mesma, como pode-se encontrar em Fienberg(1980), Agresti(1984), Andersen(1991) dentre outros.

Poucas técnicas permitem a verificação de algum tipo de associação entre as linhas ou entre as colunas da tabela, (Goodman,1985), como entre as espécies do exemplo, do ponto de vista de suas preferências por ambientes, este é um problema muito importante em áreas biológicas como a ecologia, por exemplo.

Além disso, é importante verificar se as espécies se atraem (ou se repelem) por si mesmas ou através de ligações em grupos, ou seja, estudar se há evidência de associação entre os níveis das categorias e a possibilidade de ajustar-se um modelo explicativo para um nível em função dos outros níveis da mesma (categoria). Em muitas situações práticas pode ser necessário propor as relações causais entre os níveis de uma categoria como sendo os efeitos dos outros níveis da mesma 
categoria.

No contexto descrito, propõe-se utilizar os parâmetros de dependência-co-dependência (Cordeiro, 1990) para se transpor a teoria de análise por caminhos para variáveis quantitativas (Wright, 1934; Li, 1975; Achcar, 1976; Wermuth, 1980) às tabelas de contingência de dupla entrada.

Com a finalidade de ilustrar os resultados teóricos obtidos, é feita uma aplicação a dados reais . Para esse fim, foram usados os dados de um estudo de associação entre Drosophyla e Leveduras, quanto à sua preferência alimentar (Bélo \& Lavaca, 1982). Tanto por uma atração direta (delas) quanto por atração indireta através de outras espécies. 


\title{
Path Dependency Analysis
}

\author{
Author: Doris Gómez Ticerán \\ Adviser: Prof. Dr. Antônio Cordeiro
}

\section{SUMMARY}

A proposal for path analysis with rank variable based on codependency coefficients is presented along the lines of the methodology found in Cordeiro (1990).

Thus, if we have a table where one dimension is represented by an animal species and the other dimension by different environments, applying conventional technics it is possible to test wheter or not there is some evidence for association between the two categories and, an explanatory model is adjusted as in Fienberg(1991), Agresti (1984), Andersen (1991) and others.

Few technics allow verification for some type of association among a table rows or columns (Goodman, 1985), or among the species of the exemple above, from de point of view of the animal environmental preferences. It is very important problem, mainly in biological areas like ecology, por exemple.

In addition, it is important to verify whether species attract (or repel) each other by themselves or through groupal links. In other words, it is important to study the evidence for association among the category levels and the possibility to adjust a explanatory model for one of the levels through the others levels of the same category. In many practical situations it will be necessary to propose causal relationships among levels of a category as if they were effects of the others levels of the same category.

In this context, it is proposed to use the dependency codepen- 
dency parameters (Cordeiro, 1990) in order to transpose the theory of path analysis with quantitative variables (Wright, 1934; Li, 1975; Achcar, 1976; Wermuth, $1980)$ into the two way contingency tables.

A real data aplication is made in order to ilustrate the theoretical results obtined. In so doing, data from a study on the association between Drosophyla and yeast in relations to theirs feeding preferences were used (Bélo \& Lavaca, 1982) to verify what are the species that prefer certain yeasts, due to a direct self atraction or due to an indirect atraction through other species. 


\section{INTRODUÇÃO}

Em diversas áreas do conhecimento, como Sociologia, Psicologia, Economia, é comum a coleta de dados multivariados. Na prática encontramos duas grandes categorias de dados. As variáveis tais como o comprimento e o peso, que podem ser mensuradas quantitativamente em escala intervalar ou proporcional, designam um único valor numérico a cada observação individual e são denominadas quantitativas. Já variáveis tais como cor, forma, textura são denominadas qualitativas ou categóricas e seus níveis ou categorias são utilizados na descrição ou identificação das unidades amostrais em estudo.

No que diz respeito à escala de medida, as variáveis categóricas podem ser classificadas como ordinais ou nominais, conforme seus níveis sejam naturalmente ordenados ou não.

Frequentemente as variáveis categóricas são quantificadas utilizando algum sistema de codificação que facilite, por exemplo, subsequentes processos computacionais. O uso dos números naturais (Yates, 1948) é um sistema de escores muito utilizado. Sistemas de escores relativamente simples foram propostos por Kendall e Stuart(1967) e Goodman(1985).

Cabe ressaltar que variáveis quantitativas podem ser categorizadas; assim, por exemplo, em alguns estudos pode ser de interesse classificar indivíduos segundo o nível sócio-econômico: baixo, médio ou alto, levando em consideração sua renda total.

Os dados hipotéticos apresentados na Tabela 1 permitem esclarecer os conceitos apresentados. As variáveis medidas e suas categorias são as seguintes: 
i) $X_{1}$ :Variável nominal que descreve a "situação" em relação à menopausa ( 0 =pré-menopausa; $1=$ pós-menopausa)

ii) $X_{2}$ :Variável quantitativa que descreve a idade

iii) $X_{3}$ :Variável ordinal que descreve "disposição mental", codificada na escala de 0 a 2 ,

iv) $X_{4}$ :Variável ordinal que descreve "hostilidade", codificada na escala de 0 a 2 ,

v) $X_{5}$ :Variável ordinal que descreve "criticismo", codificada na escala de 0 a 10 .

As codificações dadas a $X_{1}, X_{3}, X_{4}$ e $X_{5}$ foram arbitrárias.

TABELA 1: Exemplo hipotético sobre a influência psico-social na incidência de câncer em mulheres

\begin{tabular}{cccccc}
\hline Mulheres & $X_{1}$ & $X_{2}$ & $X_{3}$ & $X_{4}$ & $X_{5}$ \\
\hline 1 & 1 & 49 & 0 & 2 & 3 \\
2 & 0 & 50 & 0 & 2 & 2 \\
3 & 0 & 49 & 2 & 0 & 6 \\
4 & 0 & 58 & 0 & 1 & 1 \\
5 & 1 & 46 & 1 & 1 & 1 \\
6 & 1 & 43 & 1 & 2 & 0 \\
7 & 1 & 59 & 2 & 1 & 2 \\
8 & 0 & 51 & 1 & 0 & 10 \\
9 & 0 & 50 & 0 & 0 & 0 \\
10 & 1 & 52 & 0 & 1 & 5 \\
\hline
\end{tabular}

No que diz respeito à apresentação, este tipo de dados pode ser expresso na forma de matrizes de dados individuais ou na forma de tabelas de contingência multidimensionais. Na forma matricial, é usual cada linha estar associada 
a uma unidade amostral e cada coluna a uma variável. Na forma de tabela de contingência cada variável corresponde a uma dimensão; a frequência apresentada em cada casela da tabela é a contagem das unidades amostradas com a mesma descrição ou perfil multivariado das variáveis categóricas envolvidas. A tabela de contingência é uma forma resumida de apresentação de dados usada com mais frequência.

Como um exemplo, consideremos os dados analisados por Benício(1983), correspondentes a 20878 nascimentos ocorridos em 31 maternidades do município de São Paulo em 1978. Cada nascimento foi classificado segundo três variáveis categóricas e duas categorizadas:

i) $X_{1}$ : Peso do recém-nascido

ii) $X_{2}$ : "situação" em relação à assistência pré-natal

iii) $X_{3}$ : Nível de escolaridade da mãe

iv) $X_{4}$ : Peso pré-gestacional da mãe

v) $X_{5}$ : Hábito de fumar da mãe.

Estes dados poderiam ser apresentados em uma matriz de dados individuais como constam na Tabela 2.

TABELA 2: Dados individuais de nascimentos ocorridos no Município de São Paulo, 1978

\begin{tabular}{cccccc}
\hline Nascimentos & $X_{1}$ & $X_{2}$ & $X_{3}$ & $X_{4}$ & $X_{5}$ \\
\hline 1 & $\leq 2,5 \mathrm{~kg}$ & ausente & baixo & $<50 \mathrm{~kg}$ & sim \\
2 & $\leq 2,5 \mathrm{~kg}$ & ausente & alto & $<50 \mathrm{~kg}$ & não \\
$\vdots$ & $\vdots$ & $\vdots$ & $\vdots$ & $\vdots$ & $\vdots$ \\
20878 & $>2,5 \mathrm{~kg}$ & presente & alto & $\geq 50 \mathrm{~kg}$ & não \\
\hline
\end{tabular}


Uma forma alternativa para a apresentação desses dados é a forma de tabela de contingência, como a tabela 3 a seguir:

TABELA 3: Classificação de 20878 nascimentos ocorridos no Município de São Paulo, 1978

\begin{tabular}{|c|c|c|c|c|c|c|}
\hline \multirow[t]{2}{*}{$X_{2}$} & \multirow[t]{2}{*}{$X_{3}$} & \multirow[t]{2}{*}{$X_{4}$} & \multirow[t]{2}{*}{$X_{5}$} & \multicolumn{2}{|c|}{$X_{1}$} & \multirow[t]{2}{*}{ Total } \\
\hline & & & & $<2.5$ & $\geq 2.5$ & \\
\hline \multirow[t]{8}{*}{ Ausente } & baixo & $<50 \mathrm{~kg}$ & $\operatorname{sim}$ & 67 & 249 & 316 \\
\hline & & & não & 36 & 261 & 297 \\
\hline & & $\geq 50 \mathrm{~kg}$ & $\operatorname{sim}$ & 113 & 518 & 631 \\
\hline & & & não & 45 & 721 & 766 \\
\hline & alto & $<50 \mathrm{~kg}$ & $\operatorname{sim}$ & 10 & 29 & 39 \\
\hline & & & não & 8 & 23 & 31 \\
\hline & & $\geq 50 \mathrm{~kg}$ & $\operatorname{sim}$ & 5 & 47 & 52 \\
\hline & & & não & 4 & 71 & 75 \\
\hline \multirow[t]{8}{*}{ Presente } & baixo & $<50 \mathrm{~kg}$ & $\operatorname{sim}$ & 202 & 1325 & 1527 \\
\hline & & & não & 179 & 2001 & 2180 \\
\hline & & $\geq 50 \mathrm{~kg}$ & $\operatorname{sim}$ & 219 & 2655 & 2874 \\
\hline & & & não & 249 & 5665 & 5914 \\
\hline & alto & $<50 \mathrm{~kg}$ & $\operatorname{sim}$ & 59 & 525 & 584 \\
\hline & & & não & 65 & 991 & 1056 \\
\hline & & $\geq 50 \mathrm{~kg}$ & $\operatorname{sim}$ & 77 & 1408 & 1485 \\
\hline & & & não & 93 & 2958 & 3051 \\
\hline Total & & & & 1431 & 19447 & 20878 \\
\hline
\end{tabular}

Um outro ângulo de interesse na classificação das variáveis que definem uma tabela de contingência é a distinção entre as variáveis respostas e as variáveis explicativas. As variáveis respostas (variáveis dependentes) são aleatórias e descrevem o que foi observado nas unidades amostrais durante o experimento ou 
pesquisa considerada. As variáveis explicativas (independentes ou fatores) são fixas, por planejamento ou condicionamento, e as combinações de seus níveis definem as populações ou subpopulações às quais pertencem as unidades amostrais. A classificação de uma variável como resposta ou explicativa depende fundamentalmente do esquema de amostragem e do objetivo da pesquisa. Em função dessas classificações definem-se dois tipos básicos de tabelas de contingência:

i) A: tabelas em que algumas variáveis são explicativas e outras são respostas,

ii) B: tabelas onde todas as variáveis consideradas são respostas.

Em tabelas do Tipo A o objetivo, em geral, é estudar os efeitos das variáveis explicativas na distribuição conjunta das variáveis respostas ou sobre alguma característica específica dessas distribuições, como uma medida de associação entre as variáveis resposta, por exemplo. Um caso particular de grande importância é aquele em que consideramos só uma variável como resposta e as demais como variáveis explicativas. Neste caso como em Análise de Variância (ANOVA), o objetivo é estudar a influência isolada ou combinada das variáveis explicativas na distribuição da variável resposta.

Em tabelas do Tipo B, o interesse frequentemente é dirigido ao estudo de associação entre as diversas variáveis resposta, como por exemplo, para a verificação da existência de algum grau de dependência estocástica entre as variáveis. O termo associação neste caso, tem significado similar ao termo correlação, utilizado no estudo da relação existente entre variáveis estocásticas quantitativas. Consequentemente, quando existe associação entre as variáveis respostas, é de interesse avaliar o seu grau entre as variáveis envolvidas, o que se faz através dos modelos de associação.

Como exemplos consideremos as Tabelas 4 e 5 apresentadas e analisadas em Dobson(1990) e a Tabela 6 já bem analisada por Cordeiro(1990) e outros autores.

A Tabela 4 refere-se à classificação de 400 pessoas segundo o tipo de câncer de pele e o local da lesão, o objetivo da pesquisa foi estudar a intensidade 
da associação entre essas variáveis. Têm-se duas variáveis nominais respostas: tipo histológico de câncer e local da lesão.

TABELA 4: Classificação de pessoas com câncer de pele segundo o tipo de câncer e o local da lesão

\begin{tabular}{ccccc}
\hline Tipo de Cancer & \multicolumn{4}{c}{ Local da Lesão } \\
& Cabeça e Pescoço & Tronco & Extremidades & Total \\
\hline A & 22 & 2 & 10 & 34 \\
B & 16 & 54 & 115 & 185 \\
C & 19 & 33 & 73 & 125 \\
D & 11 & 17 & 28 & 56 \\
\hline Total & 68 & 106 & 226 & 400 \\
\hline
\end{tabular}

Os dados da tabela 5 referem-se a um estudo retrospectivo para verificar o efeito de uma nova vacina contra gripe. Para isto, em 38 pacientes foi aplicada a vacina e em outros 35 , placebo. Seis semanas após a vacinação foi feita uma classificação do número de pessoas com nível baixo, médio e alto de anticorpo inibidor hemoglutina (HIA) encontrado no sangue. Tem-se nesse caso uma variável explanatória (o tratamento vacina ou placebo) que é nominal e uma variável resposta (nível de HIA) que é ordinal.

TABELA 5: Classificação de pacientes segundo o tipo de vacina e níveis de anticorpo hemoglutina encontrado no sangue

\begin{tabular}{ccccc}
\hline & \multicolumn{4}{c}{ Níveis de Anticorpo } \\
Droga & Baixo & Médio & Alto & Total \\
\hline Placebo & 25 & 8 & 5 & 38 \\
Vacina & 6 & 18 & 11 & 35 \\
\hline
\end{tabular}

Os dados da Tabela 6 referem-se à classificação de 1660 indivíduos 
cujo objetivo foi estudar a associação existente entre a saúde mental e a condição socio-econômica dos pais; as duas variáveis envolvidas são do tipo ordinal.

TABELA 6: Classificação de 1660 pessoas segundo a saúde mental e o estado sócio -econômico dos pais

\begin{tabular}{lccccccc}
\hline & \multicolumn{7}{c}{ Estado sócio-econômico dos pais } \\
Saúde mental & A & B & C & D & E & F & Total \\
\hline Boa & 64 & 57 & 57 & 72 & 36 & 21 & 307 \\
F.S.Suave & 94 & 94 & 105 & 141 & 97 & 71 & 602 \\
F.S.Moderada & 58 & 54 & 65 & 77 & 54 & 54 & 362 \\
Má & 46 & 40 & 60 & 94 & 78 & 71 & 389 \\
\hline Total & 262 & 245 & 287 & 384 & 265 & 217 & 1660 \\
\hline
\end{tabular}

Em análise de tabelas de contingência de dados categóricos, poucas são as medidas de associação entre linhas, entre colunas e entre linhas e colunas. A maior parte delas vêm da interpretação de parâmetros em modelos que se ajustam aos dados. Têm-se, por exemplo, os modelos log-lineares (Bishop, Fienberg e Holland, 1975), os modelos de associação de Goodman (Goodman, 1985), dos mínimos quadrados ponderados (Grizzle, Starmer e Koch, 1969), além da análise de correspondência (Lebart, Morineau e Fénelon, 1982; Greenacre, 1984).

Como tese de Livre-docência, em 1990 Cordeiro (Cordeiro, 1990) propôs a análise de dependência-ANADEP para estudar associações nestas tabelas. A proposta constou de vários parâmetros que emulam, para o caso de dados categóricos, aqueles de variáveis aleatórias numéricas como variâncias, covariâncias e coeficiente de correlação.

O emulador do coeficiente de correlação é o coeficiente de co-dependência que mede, tanto no espaço das linhas quanto no das colunas da tabela, o grau de similaridade entre dois níveis da categoria de interesse. Este coeficiente, 
como o de correlação, varia entre -1 e 1 , valendo 1 quando os dois níveis forem exatamente iguais como distribuições probabilísticas condicionais, zero quando não houver associação entre eles e tende a -1 quanto maior for a associação no sentido negativo, visto como distribuições de probabilidade condicionais, onde um tem valor alto o outro o tem baixo, e vice-versa (Cordeiro, 1990).

A medida de associação entre variáveis aleatórias numéricas que mais tem sido usada é a correlação que, com suas variações já está bem explorada, assim como suas generalizações, como o coeficiente de correlação parcial e coeficiente de correlação múltipla.

Da necessidade da decomposição do coeficiente de correlação foi proposto o coeficiente de trajetórias ("PATH COEFFICIENTS"), cujo estudo e aplicações geraram a Análise de Trajetórias ou Caminhos ("PATH ANALYSIS"), (Wright, 1918; Achcar, 1976; Dillon e Goldstein, 1984; Li, 1975 entre outros).

Os objetivos do presente trabalho referem-se a criação da Análise de Trajetórias para dados Categóricos com base na análise de dependência-ANADEP. Assim, será possível:

i) Ajustar um modelo explicativo para um nível de uma das categorias da tabela, através dos outros níveis da mesma categoria;

ii) A generalização para a análise de dados categóricos, de uma técnica já existente e bem utilizada em análise de correlações de variáveis aleatórias numéricas;

iii) Estimar os coeficientes de co-dependências e decompor por trajetórias em seus efeitos diretos e indiretos, emulando-se a teoria de análise de trajetória para variáveis numéricas, com base no modelo amostral multinomial. Será demonstrado um teorema, denominado Teorema Fundamental de Análise de Trajetória que reproduz o caso contínuo para variáveis numéricas;

iv) Testar hipóteses para os coeficientes de trajetória e propor um melhor modelo explicativo para um nível de uma das categorias, através dos outros níveis da mesma categoria; 
v) Uma aplicação a dados reais dos resultados teóricos obtidos, para o qual serão usados os dados de um estudo de associação entre Drosophyla e Leveduras, quanto à preferência alimentar (Bélo \& Lavaca, 1982), com a finalidade de verificar quais são as espécies que preferem certas leveduras, por uma atração direta delas ou por atração indireta através de outras espécies.

Para atingir os objetivos propostos, a seguir apresentam-se alguns resultados das Teorias ANADEP e Análise de Trajetória para Variáveis Numéricas, a fim de facilitar o desenvolvimento da Análise de Trajetória para Dados Categóricos. 


\section{REVISÃO BIBLIOGRÁFICA}

Neste capítulo apresenta-se a primeira parte da análise de dependência- ANADEP (Cordeiro, 1990), pois como já foi dito, a proposta da análise de trajetória para tabelas de contingência está baseada nos resultados que fornecem esta teoria e é fundamental o seu entendimento. Também são apresentados resultados importantes da análise da trajetória para variáveis numéricas.

\subsection{Análise de dependência e co-dependência populacional}

Sejam A e B duas características de classificação de indivíduos de uma população (suposta infinita), com $A_{1}, \ldots, A_{I}$ e $B_{1}, \ldots, B_{J}$ sendo os níveis de $\mathrm{A}$ e $\mathrm{B}$, respectivamente. Define-se:

$$
\mathbf{P}=\left[p_{i j}\right]
$$

a matriz de probabilidades, onde $i=1, \ldots, I ; j=1, \ldots, J$

$$
p_{i j}=P\left(A_{i} \cap B_{j}\right)
$$

é a probabilidade de um indivíduo escolhido ao acaso desta população pertencer aos níveis $i$ e $j$ das características $\mathrm{A}$ e $\mathrm{B}$, respectivamente.

Supondo-se $p_{i j}>0$, para todo par $(i, j)$ e fazendo-se:

$$
\begin{aligned}
& p_{i .}=\sum_{j=1}^{J} p_{i j}, \quad \text { para } 1 \leq i \leq I \\
& p_{. j}=\sum_{i=1}^{I} p_{i j}, \quad \text { para } 1 \leq j \leq J,
\end{aligned}
$$




$$
\sum_{i=1}^{I} \sum_{j=1}^{J} p_{i j}=1
$$

então os vetores linha

$$
\mathbf{p}_{r, i}^{\prime}=\left(p_{i 1}, p_{i 2}, \ldots, p_{i J}\right) / p_{i .} \quad 1 \leq i \leq I
$$

e os vetores coluna

$$
\mathbf{p}_{c, j}^{\prime}=\left(p_{1 j}, p_{2 j}, \ldots, p_{I j}\right) / p_{. j} \quad 1 \leq j \leq J
$$

são distribuições de probabilidades. Também o é o vetor linha marginal,

$$
\mathbf{p}_{r,+}^{\prime}=\left(p_{.1}, p_{.2}, \ldots, p_{. J}\right) ; \text { labeleq } 5
$$

e o vetor coluna marginal,

$$
\mathbf{p}_{c,+}^{\prime}=\left(p_{1 .}, p_{2 .}, \ldots, p_{I .}\right)
$$

Estes vetores são denominados perfis probabilísticos ou somente perfis.

Com as anotações anteriores, que são as mesmas de ANADEP, colocadas na forma matricial tem-se a matriz de probabilidades e perfis

$$
\begin{gathered}
\mathbf{P}=\left(\begin{array}{cccc}
p_{11} & p_{12} & \ldots & p_{1 J} \\
p_{21} & p_{22} & \ldots & p_{2 J} \\
\vdots & \vdots & \vdots & \vdots \\
p_{I 1} & p_{I 2} & \ldots & p_{I J}
\end{array}\right) \\
\mathbf{p}_{c,+}=\left(\begin{array}{c}
p_{1 .} \\
p_{2 .} \\
\vdots \\
p_{I .}
\end{array}\right) \\
\mathbf{p}_{r,+}=\left(\begin{array}{c}
p_{.1} \\
p_{.2} \\
\vdots \\
p_{. J}
\end{array}\right)
\end{gathered}
$$




$$
\mathbf{p}_{c, j}=\left(\begin{array}{c}
p_{1 j} / p_{. j} \\
\vdots \\
p_{I j} / p_{. j}
\end{array}\right)
$$

$$
\mathbf{p}_{r, i}=\left(\begin{array}{c}
p_{i 1} / p_{i .} \\
\vdots \\
p_{i J} / p_{i .}
\end{array}\right)
$$

a matriz $\Delta$

$$
\Delta=\frac{1}{\sqrt{2}}\left(\sqrt{\mathbf{P}}-\sqrt{\mathbf{p}_{c,+} \mathbf{p}_{r,+}^{\prime}}\right)
$$

de ordem IJ,

$$
\begin{aligned}
& \Delta=\frac{1}{\sqrt{2}}\left(\begin{array}{cccc}
\sqrt{p_{11}} & \sqrt{p_{12}} & \cdots & \sqrt{p_{1 J}} \\
\sqrt{p_{21}} & \sqrt{p_{22}} & \cdots & \sqrt{p_{2 J}} \\
\vdots & \vdots & \vdots & \vdots \\
\sqrt{p_{I 1}} & \sqrt{p_{I 2}} & \cdots & \sqrt{p_{I J}}
\end{array}\right) \\
& -\left(\begin{array}{c}
\sqrt{p_{1 .}} \\
\sqrt{p_{2 .}} \\
\vdots \\
\sqrt{p_{I .}}
\end{array}\right)\left(\begin{array}{cccc}
\sqrt{p_{.1}} & \sqrt{p_{.2}} & \cdots & \sqrt{p_{. J}}
\end{array}\right) \\
& \Delta=\frac{1}{\sqrt{2}}\left(\begin{array}{cccc}
\sqrt{p_{11}} & \sqrt{p_{12}} & \cdots & \sqrt{p_{1 J}} \\
\sqrt{p_{21}} & \sqrt{p_{22}} & \cdots & \sqrt{p_{2 J}} \\
\vdots & \vdots & \vdots & \vdots \\
\sqrt{p_{I 1}} & \sqrt{p_{I 2}} & \cdots & \sqrt{p_{I J}}
\end{array}\right)-\left(\begin{array}{ccc}
\sqrt{p_{1 . p_{.1}}} & \cdots & \sqrt{p_{1 . p_{. J}}} \\
\sqrt{p_{2 . p_{.1}}} & \cdots & \sqrt{p_{2 . p_{. J}}} \\
\vdots & \vdots & \vdots \\
\sqrt{p_{.1}} & \cdots & \sqrt{p_{I .1} p_{. J}}
\end{array}\right) \\
& \Delta=\frac{1}{\sqrt{2}}\left(\begin{array}{ccc}
\sqrt{p_{11}}-\sqrt{p_{1 . p_{.1}}} & \cdots & \sqrt{p_{1 J}}-\sqrt{p_{1 . p_{. J}}} \\
\vdots & \vdots & \vdots \\
\sqrt{p_{I 1}}-\sqrt{p_{I . p_{.1}}} & \vdots & \sqrt{p_{I J}}-\sqrt{p_{I . p_{. J}}}
\end{array}\right)
\end{aligned}
$$


a matriz de dependências e co-dependências entre as colunas

$$
D_{c}=\Delta^{\prime} \Delta=\left(\begin{array}{ccc}
d_{c, 11} & d_{c, 12} & d_{c, 1 J} \\
d_{c, 21} & d_{c, 22} & d_{c, 2 J} \\
\vdots & \vdots & \vdots \\
d_{c, J 1} & d_{c, J 2} & d_{c, J J}
\end{array}\right)
$$

que contém as dependências e co-dependências entre as colunas $j$ e $j *, j \neq j *$, onde

$$
\begin{aligned}
& d_{c, j j}=\frac{1}{2} \sum_{i=1}^{I}\left(\sqrt{p_{i j}}-\sqrt{p_{i . p_{. j}}}\right)^{2} \\
& d_{c, j j *}=\frac{1}{2} \sum_{i=1}^{I}\left(\sqrt{p_{i j}}-\sqrt{p_{i . p_{. j}}}\right)\left(\sqrt{p_{i j *}}-\sqrt{p_{i . p_{. j *}}}\right) ;
\end{aligned}
$$

a matriz de coeficientes de co-dependências entre as colunas,

$$
\Upsilon_{c}=\left(\begin{array}{cccc}
1 & \delta_{c, 12} & \ldots & \delta_{c, 1 J} \\
\delta_{c, 21} & 1 & \ldots & \delta_{c, 2 J} \\
\vdots & \vdots & \vdots & \vdots \\
\delta_{c, J 1} & \delta_{c, J 2} & \vdots & 1
\end{array}\right)
$$

que contém os coeficientes de co-dependências entre as colunas $j$ e $j *, j \neq j *$, onde

$$
\delta_{c, j j *}=d_{c, j j *} /\left(d_{c, j j} d_{c, j * j *}\right)^{1 / 2}
$$

e

$$
\delta_{c, j j}=1
$$

a matriz de dependências e co-dependências para as linhas é dada por

$$
D_{r}=\Delta \Delta^{\prime}=\left(\begin{array}{cccc}
d_{r, 11} & d_{r, 12} & \ldots & d_{r, 1 I} \\
d_{r, 21} & d_{r, 22} & \ldots & d_{r, 2 I} \\
\vdots & \vdots & \vdots & \vdots \\
d_{r, I 1} & d_{r, I 2} & \ldots & d_{r, I I}
\end{array}\right)
$$


que contém as dependências, $d_{r, i i}$ e co-dependências $d_{r, i i *}$ entre as linhas $i$ e $i *$, $i \neq i *$, onde

$$
\begin{aligned}
& d_{r, i i}=\frac{1}{2} \sum_{j=1}^{J}\left(\sqrt{p_{i j}}-\sqrt{p_{i . p_{. j}}}\right)^{2} \\
& d_{r, i i *}=\frac{1}{2} \sum_{j=1}^{J}\left(\sqrt{p_{i j}}-\sqrt{p_{i . p_{. j}}}\right)\left(\sqrt{p_{i * j}}-\sqrt{p_{i * .} p_{. j}}\right) ;
\end{aligned}
$$

a matriz de coeficientes de co-dependências entre as linhas

$$
\Upsilon_{r}=\left(\begin{array}{cccc}
1 & \delta_{r, 12} & \ldots & \delta_{r, 1 I} \\
\delta_{r, 21} & 1 & \ldots & \delta_{r, 2 I} \\
\vdots & \vdots & \vdots & \vdots \\
\delta_{r, I 1} & \delta_{r, I 2} & \ldots & 1
\end{array}\right)
$$

que contém os coeficientes de co-dependências, entre as linhas $i$ e $i *, i \neq i *$, onde

$$
\delta_{r, i i *}=d_{r, i i *} /\left(d_{r, i i} d_{r, i * i *}\right)^{1 / 2}
$$

e

$$
\delta_{r, i i}=1
$$

Os parâmetros, elementos das matrizes $D_{c}$ e $D_{r}$, têm interpretações e algumas propriedades semelhantes às variâncias e às covariâncias, enquanto os elementos das matrizes $\Upsilon_{c}$ e $\Upsilon_{r}$ têm interpretações e algumas propriedades semelhantes aos coeficientes de correlaçōes para variáveis aleatórias. Assim;

1) A co-dependência (17) entre as colunas $j$ e $j *$ é proporcional ao produto interno entre os desvios das raízes quadradas dos perfis probabilísticos $\mathbf{p}_{c, j}$ e $\mathbf{p}_{c, j *}$ em relação à raiz quadrada do perfil probabilístico marginal $\mathbf{p}_{c,+}$, a saber:
i) $d_{c, j j *}=\frac{1}{2} \sum_{i=1}^{I}\left(\sqrt{p_{i j}}-\sqrt{p_{i . p_{. j}}}\right)\left(\sqrt{p_{i j *}}-\sqrt{p_{i . p_{. j *}}}\right)$ 
(ii) $\mathbf{p}_{c, j}-\mathbf{p}_{c,+}=\left(\begin{array}{c}p_{1 j} / p . j \\ p_{2 j} / p . j \\ \vdots \\ p_{I j} / p_{. j}\end{array}\right)-\left(\begin{array}{c}p_{1 .} \\ p_{2 .} \\ \vdots \\ p_{I .}\end{array}\right)$

$=\left(\begin{array}{c}\left(p_{1 j}-p_{1 .} p_{. j}\right) / p_{. j} \\ \left(p_{2 j}-p_{2 . p_{j}}\right) / p_{. j} \\ \vdots \\ \left(p_{I j}-p_{I .} p_{. j}\right) / p_{. j}\end{array}\right)$

(iii) $\mathbf{p}_{c, j *}-\mathbf{p}_{c,+}=\left(\begin{array}{c}p_{1 j *} / p_{. j *} \\ p_{2 j *} / p_{. j *} \\ \vdots \\ p_{I, j *} / p_{. j *}\end{array}\right)-\left(\begin{array}{c}p_{1 .} \\ p_{2 .} \\ \vdots \\ p_{I .}\end{array}\right)$

$=\left(\begin{array}{c}\left(p_{1 j *}-p_{1 .} p_{. j *}\right) / p_{. j *} \\ \left(p_{2 j *}-p_{2 . p_{. j *}}\right) / p_{. j *} \\ \vdots \\ \left(p_{I j *}-p_{I .} p_{. j *}\right) / p_{. j *}\end{array}\right)$

(iv) $\left(\sqrt{\mathbf{p}_{c, j}}-\sqrt{\mathbf{p}_{c,+}}\right)^{\prime}\left(\sqrt{\mathbf{P}_{c, j *}}-\sqrt{\mathbf{p}_{c,+}}\right)$

$=\frac{1}{\sqrt{p_{. j} p_{. j *}}} \sum_{i=1}^{I}\left(\sqrt{p_{i j}}-\sqrt{p_{i . p_{. j}}}\right)\left(\sqrt{p_{i j *}}-\sqrt{p_{i . p_{. j *}}}\right)$

$=\frac{1}{2} \sum_{i=1}^{I}\left(\sqrt{p_{i j}}-\sqrt{p_{i . p_{. j}}}\right)\left(\sqrt{p_{i j *}}-\sqrt{p_{i .} p_{. j *}}\right) \frac{2}{\sqrt{p_{. j} p_{. j *}}}$

$=d_{c, j j *} \frac{2}{\sqrt{p_{. j} p_{. j *}}}$. 
Logo, $d_{c, j j *}$ é proporcional a $\left(\sqrt{\mathbf{P}_{c, j}}-\sqrt{\mathbf{P}_{c,+}}\right)^{\prime}\left(\sqrt{\mathbf{P}_{c, j *}}-\sqrt{\mathbf{P}_{c,+}}\right)$. Da mesma maneira demonstra-se que para as linhas, $d_{r, i i *}$, é proporcional a $\left(\sqrt{\mathbf{P}_{r, i}}-\right.$ $\left.\sqrt{\mathbf{P}_{r,+}}\right)^{\prime}\left(\sqrt{\mathbf{P}_{r, i *}}-\sqrt{\mathbf{p}_{r,+}}\right)$.

Para analisar uma semelhança entre a co-dependência e a covariância é necessária a definição a seguir.

Definição 1 .- A coluna $\mathrm{j}$, ou o nível $B_{j}$, onde $1 \leq j \leq J$, da categoria B da tabela, é independente das linhas, ou da categoria $\mathrm{A}$, se

$$
p_{i j}=p_{i .} p_{. j}
$$

para todo $i=1, \ldots, I$; ou seja

$$
\begin{gathered}
\left(\begin{array}{c}
p_{1 j} \\
p_{2 j} \\
\vdots \\
p_{I j}
\end{array}\right)=p_{. j}\left(\begin{array}{c}
p_{1 .} \\
p_{2 .} \\
\vdots \\
p_{I .}
\end{array}\right) \\
\left(\begin{array}{c}
p_{1 j} / p_{. j} \\
p_{2 j} / p_{. j} \\
\vdots \\
p_{I j} / p_{. j}
\end{array}\right)=\left(\begin{array}{c}
p_{1 .} \\
p_{2 .} \\
\vdots \\
p_{I .}
\end{array}\right)
\end{gathered}
$$

isto é,

$$
\mathbf{p}_{c, j}=\mathbf{p}_{c,+}
$$

Esta definição traz em tabelas de contingência o conceito de independência das categorias A e B para os níveis de uma em relação à outra. Se todos os níveis de uma categoria forem independentes da outra, então elas são independentes, o que é evidente por (27).

Com esta conceituação, um resultado semelhante aos existentes para a covariância entre variáveis numéricas é o seguinte: 
2) Se a coluna $j$ for independente da coluna $j *$, segundo as linhas, então, $d_{c, j j_{*}}=$ 0 , para todo $j \neq j *$. Isto quer dizer que o perfil coluna $\mathbf{p}_{c, j}$ não se diferencia do perfil marginal $\mathbf{p}_{c,+}$, isto é , o nível $B_{j}$ da categoria B não é diferente segundo a categoria $\mathrm{A}$, da marginal. Quando isto acontece, o nível $B_{j}$ pode ser ignorado do estudo de associação entre as categorias A e B. A saber,

$$
\begin{aligned}
& d_{c, j j^{*}}=\frac{1}{2} \sqrt{p_{j} p_{. j *}}\left(\sqrt{\mathbf{P}_{c, j}}-\sqrt{\mathbf{P}_{c,+}}\right)^{\prime}\left(\sqrt{\mathbf{P}_{c, j *}}-\sqrt{\mathbf{P}_{c,+}}\right) \\
& =\frac{1}{2} \sqrt{p_{. j} p_{. j *}}\left(\sqrt{\mathbf{P}_{c,+}}-\sqrt{\mathbf{P}_{c,+}}\right)^{\prime}\left(\sqrt{\mathbf{P}_{c,+}}-\sqrt{\mathbf{P}_{c,+}}\right) \\
& =0
\end{aligned}
$$

e, consequentemente

$$
\delta_{c, j j *}=0 \quad \text { para todo } j * .
$$

Há situações em que $d_{c, j j *}=0$, mas a coluna $j$ e a coluna $j *$, são associadas, da mesma maneira que covariância nula não garante a independência estocástica. O valor positivo de $d_{c, j j *}$ significa que as probabilidades dos perfis-coluna $j$ e $j *$ tendem a estar do mesmo lado quando comparados com as probabilidades do perfil marginal, o valor negativo indica tendência contrária. Isto é, se condicionada à coluna $j$, a probabilidade de um nível $i$ da categoria A é maior que sua probabilidade marginal, e condicionada à coluna $j *$, o valor da probabilidade condicional de $A_{i}$ tende a ser menor que o da marginal, e vice-versa.

Se a co-dependência $d_{c, j j *}$ não é nula, o quanto ela é positiva ou negativa vai depender também dos pesos marginais das colunas $j$ e $j *$; da mesma forma, se a coluna $j$ for dependente das linhas, sua dependência $d_{c, j j}$ será proporcional ao seu peso marginal, isto é, colunas com pesos marginais maiores tendem a serem mais dependentes (e importantes no estudo de associação) das linhas.

Os coeficientes de co-dependência (19) e (25) eliminam, como medida de associação, a influência dos pesos marginais, assim como o coeficiente de correlação elimina o efeito das dispersões para variáveis aleatórias numéricas e valem as proposições: 
Proposição 1: $\mathrm{O}$ valor absoluto de $\delta_{c, j j *}$ é no máximo igual a 1.

Proposição 2: Supondo $\delta_{c, j j}>0$ e $\delta_{c, j * j *}>0$. Então $\delta_{c, j * j *}=1$ se e somente se, $\mathbf{p}_{c, j}=\mathbf{p}_{c, j *}$.

Definição 2 .- A dependência total da tabela de contingência é definida como

$$
\mathrm{d}^{2}=\sum_{i=1}^{I} d_{r, i i}=\sum_{j=1}^{J} d_{c, j j}
$$

onde $\mathbf{d}^{2}$ é uma medida de associação global na tabela de contingência.

Se cada coluna for independente das linhas, por (22) e (27) a dependência total da tabela será nula e as categorias A e B serão independentes e consequentemente, qualquer linha será independente das colunas.

Maiores detalhes teóricos, demonstrações e a construção de componentes principais em tabelas bidimensionais, são apresentados em Cordeiro(1990).

\subsection{Análise de trajetória numérica}

\subsubsection{Considerações gerais}

A análise de trajetória ou a análise de caminhos para variáveis aleatórias numéricas aparece pela primeira vez em Wright( 1918), dando continuidade a essas aplicações em anos posteriores. Referências importantes deste assunto com ilustrações importantes encontram-se em Wright(1934) e Wright(1960). $\mathrm{O}$ autor introduziu esse procedimento a partir de diagramas genéticos para analisar problemas de cruzamentos. Uma abordagem elementar é apresentada em $\mathrm{Li}(1975)$.

A técnica teve grande êxito inicial e sofreu também algumas críticas sobre as interpretações de resultados em genética, às quais Wright(1934) respondeu satisfatoriamente.

Tukey(1954) fez uma crítica sobre o cálculo dos coeficientes de trajetória a partir dos coeficientes de correlação, uma vez que considerava este como parte de análise descritiva e os coeficientes de trajetória como parte de análise de 
regressão, por sua estrutura funcional. Esta distinção provocou muitas discussões que duram até hoje, fazendo com que os modelos causais sejam ajustados pelo uso de métodos de regressão e análises não causais possam ser feitas por procedimentos de análise de trajetória com base nas correlações.

A análise por caminhos tem sido criticada como querendo estabelecer relações de causa e efeito, se bem que Wright nunca a promoveu como um método para descobrir causas. Como observou ele:

Wright(1934, p 193):"... o método de coeficientes por caminhos não pretende engajamento na impossível tarefa de dedução de relações causais a partir de valores de coeficientes de correlação. Ele pretende combinar a informação quantitativa dada pelas correlações com as informações qualitativas que possam estar às mãos em relações causais para dar uma interpretação quantitativa".

Análise de trajetória é um método útil para estudar um sistema de variáveis interrelacionadas, com base na construção de um diagrama qualitativo. Assim, análise de trajetória é um método para estudar as estruturas de causalidade entre um conjunto de variáveis. O método foi popularizado na literatura sociológica por Blalock(1961) e Duncan(1966), dentre outros. A construção de modelos recursivos lineares usando-se a análise de trajetória e a regressão múltipla é reconhecido como uma abordagem útil para quantificar e interpretar relações causais no campo da sociologia.

Também, análises não causais entre um conjunto de variáveis podem ser feitas por procedimentos de análise de trajetória baseando-se na estrutura de correlações das variáveis envolvidas.

Para a aplicação do método de análise de trajetória precisa-se inicialmente considerar um diagrama de trajetórias, associado às variáveis do problema em questão, no qual as interrelações entre as variáveis incluídas mensuráveis ou hipotéticas são representadas por setas. Esse diagrama é construído pelo pesquisador considerando-se algumas variáveis como efeitos e outras variáveis como causas, com base nas considerações teóricas da área de aplicação (Wright, 1918; Achcar, 
1976 ; Wermuth, 1980; Dillon \& Goldstein, 1984; Glymour, 1993; e outros).

$\mathrm{Na}$ literatura estatística existem duas tentativas para a representação matemática de dependência causal entre um conjunto de variáveis (Glymour, 1993).

O primeiro que usou os grafos dirigidos para representar estruturas causais foi Wright (1954). Ocasionalmente esses grafos têm sido usados nas interpretações de modelos de regressão, modelos de análise de fatores, modelos de equações simultâneas e outros. Do ponto de vista matemático, os grafos dirigidos estão implícitos toda vez que o modelo estatístico está caracterizado por um conjunto de equações algébricas, onde uma única variável em um lado da equação é tratada como efeito das variáveis que estão no outro lado da equação. Um caso interessante resulta quando existem algumas condições que permitam relacionar as estruturas gráficas com relações probabilísticas.

Algumas vezes consideram-se explicitamente relações sobre correlações e correlações parciais (Simon, 1954; Blalock, 1961), que no caso de normalidade são as mesmas condições de independência e independência condicional.

Sem usar grafos, alguns filósofos notáveis e entre eles Suppes (1970) tentaram analisar a noção de causalidade em termos de dependência estatística, dando uma ordenação temporal. Mas, em uma abordagem geral conectando explicitamente grafos dirigidos e distribuições probabilísticas foram formuladas idéias de dependência causal na denominada condição de Markov, por Kiiveri e Speed (1982). Para uma estrutura causal a condição de Markov fornece uma fatoração da distribuição de probabilidade em termos da probabilidade condicional de cada variável dadas suas causas.

Desta forma, a prática muito difundida em aplicações estatísticas recebeu uma elegante base formal, conectando trabalhos estatísticos com respeito à fatoração de distribuições que previamente tinham sido abordadas por Wermuth(1980) e outros autores. Consequências adicionais sobre a condição de Markov e restrições adicionais sobre a conecção entre grafos dirigidos e distribuições foram 
subsequentemente desenvolvidos por muitos autores e entre eles estão Wermuth \& Lauritzen(1983); Lauritzen \& Wermuth(1989); Wermuth \& Lauritzen (1990); Wittaker(1990); Cox(1992); Lauritzen(1989); Cox \& Wermuth(1993).

Uma outra linha de pesquisa que conecta probabilidade e causalidade e dá ênfase às relações entre a noção de causalidade e os efeitos de manipulação, surgiu de trabalhos em delineamento experimental. Rubin(1974, 1977, 1990) e vários outros autores entre eles Holland(1986), Pratt \& Schlaifer(1988) interpretam hipóteses causais para o que consideram que causa e tratamento têm o mesmo significado. Enfatizam a importância da aleatorização no delineamento experimental para estimar os efeitos causais de uma variável sobre uma outra variável.

Em um modelo estrutural causal, determinam-se à prióri as variáveis (latentes ou manifestas) dependentes e quais são as independentes, isto é as variáveis efeitos e as variáveis causas. Em geral, as técnicas de modelagem causal não permitem que se determine a direção da causalidade, nem ao menos que se conclua a existência da relação de causalidade.

Em sua obra(Dillon \& Goldstein, 1984), observa que de pensadores clássicos como David Hume e John Stuart Mill podem identificar-se algumas condições que estabeleceriam relações causais entre duas variáveis $X$ e $Y$ :

i) Deve haver variação concomitante ou covariação entre $\mathrm{X}$ e Y,

ii) Deve existir assimetria cronológica (ordenação no tempo) entre $\mathrm{X}$ e Y,

iii) A covariância entre $\mathrm{X}$ e $\mathrm{Y}$ não deve desaparecer, quando os efeitos de outras variáveis causalmente antecedentes a $\mathrm{X}$ e Y (isto é, variáveis de confundimento) são controladas.

Na prática, as duas primeiras condições não são problemáticas, mas a terceira sim, uma vez que requer a eliminação de todos os fatores possíveis e assim, o problema é identificá-los.

Em um modelo causal, as variáveis que causam outras e cujas variabilidades são assumidas serem causadas por outras variáveis fora do modelo são 
chamadas exógenas. As variáveis que são explicadas pelas exógenas, bem como outras do modelo são chamadas endógenas.

O uso efetivo de análise de trajetória ou de caminhos é estabelecido sob algumas suposições. As mais importantes são:

i) Os modelos de análise por caminhos assumem que os relacionamentos entre as variáveis mensuráveis são lineares e aditivos; modelos curvilineares e multiplicativos são excluídos. Embora, algumas das variáveis sejam mensuráveis, outras são hipotéticas como, por exemplo, os "fatores" em análise fatorial;

ii) Todos os termos do erro (isto é, os resíduos) são assumidos serem não correlacionados um com o outro;

iii) Somente os modelos recursivos são considerados, isto é, aqueles que não postulam causas recíprocas entre as variáveis, ou seja, com o fluxo causal em uma só direção. Causas recíprocas entre as variáveis são proibidas;

iv) Os modelos de análise por caminhos assumem que, pelo menos, as variáveis endógenas tenham propriedades de escalas intervalares;

v) As variáveis medidas são assumidas sem erro;

vi) O modelo considerado é assumido ser corretamente especificado, isto é, que todos os determinantes causais tenham sido incluídos no modelo. Caso outros determinantes causais de variáveis endógenas não tenham sido incluídos, faz-se a suposição de que sejam independentes daqueles que já estão no modelo.

vii) Apesar de não ser intrínseca ao método, a representação gráfica é de grande utilidade.

Os aspectos inferenciais de análise de trajetória podem ser classificados, seguindo-se dois propósitos diferentes (Wermuth,1980):

1. Estimação de parâmetros em um sistema de equações lineares, 
2. Estimação dos parâmetros da correspondente matriz de correlações.

Considerando-se as suposições anteriores, os coeficientes do modelo de análise por caminhos podem ser estimados pelo método comum de mínimos quadrados. Cada equação que forma parte do modelo causal é resolvida separadamente. Esta é uma vantagem deste método, pois os erros das estimativas dos parâmetros de uma equação não afetam adversamente a estimação dos outros parâmetros que aparecem nas outras equações do sistema (Dillon \& Goldstein, 1984).

Os coeficientes por caminhos indicam o efeito direto da variável tomada como causa sobre uma variável tomada como efeito. O símbolo convencional usado para um coeficiente por caminhos será $\wp_{i j}$, onde $i$ refere-se ao efeito (variável dependente) e $j$ refere-se à causa (variável independente). Se um modelo é recursivo, então os coeficientes de caminhos podem ser expressos usando simples correlações ou regressões múltiplas.

$\mathrm{Na}$ estimação dos coeficientes por caminhos, a questão fundamental é quantas variáveis são antecedentes causais de uma variável endógena.

Se somente uma variável exógena $\mathrm{X}$ tem impacto direto sobre a variável endógena Y, como na Figura 1, então o correspondente coeficiente $\wp_{i j}=\wp_{y x}$ é estimado como a correlação simples entre X e Y, isto é ,

$$
\hat{\wp}_{i j}=r_{y x}
$$

Se $\mathrm{Y}$ tiver dois antecedentes causais $X_{1}$ e $X_{2}$, então os coeficientes para $X_{1}$ e $X_{2}$ são os pesos na regressão de $\mathrm{Y}$ sobre as duas variáveis. Observa-se na Figura 2, que

$$
\wp_{y x_{1}}=r_{y x_{1}} \quad \text { e } \wp_{y x_{2}}=r_{y x_{2}}
$$

pois $X_{1}$ e $X_{2}$ são não correlacionados.

Para melhor esclarecer como os coeficientes por caminhos podem ser calculados das correlações simples, considera-se a Figura 3, que mostra um modelo 
FIGURA 1. Uma relação causal com um antecedente

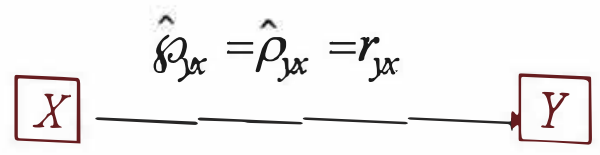

FIGURA 2. Uma relação causal com dois antecedentes não correlacionados

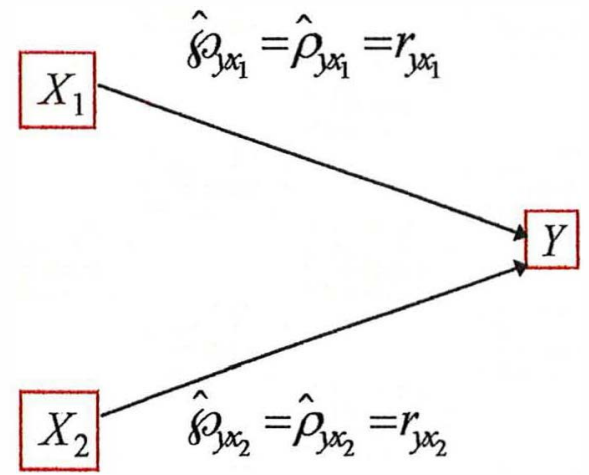

recursivo de análise por caminhos envolvendo quatro variáveis.

Expressando todas as variáveis na forma padronizada, ou seja variáveis com médias zeros e variâncias unitárias, as quatro equações correspondendo à Figura 3 são:

$$
\begin{aligned}
& X_{1}=e_{x_{1}} \quad X_{2}=e_{x_{2}} \\
& Y_{1}=\wp_{y_{1} x_{1}} X_{1}+\wp_{y_{1} x_{2}} X_{2}+e_{y_{1}} \\
& Y_{2}=\wp_{y_{2} x_{1}} X_{1}+\wp_{y_{2} x_{2}} X_{2}+\wp_{y_{2} y_{1}} Y_{1}+e_{y_{2}},
\end{aligned}
$$

onde os e's são também expressos na forma padronizada (média nula e variância unitária. Como as variáveis X's são exógenas, elas são representadas pelos resíduos 
FIGURA 3. Gráfico de caminhos com duas variáveis exógenas correlacionadas

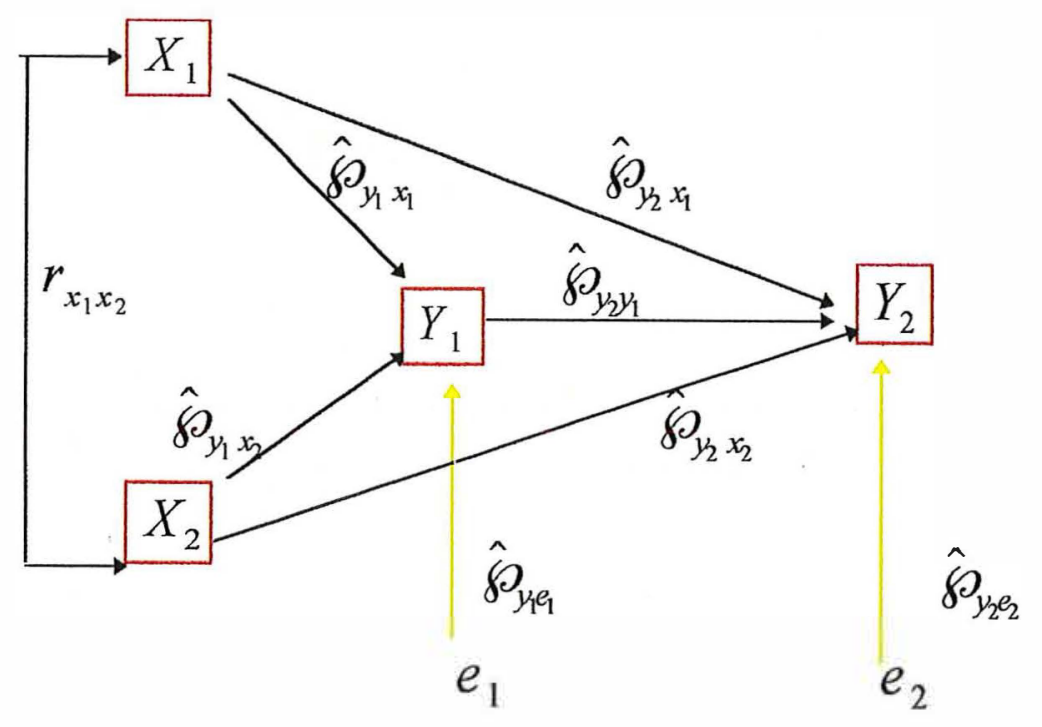

$e_{x_{1}}$ e $e_{x_{2}}$ em relação a $X_{1}$ e $X_{2}$, respectivamente. $O$ método para o cálculo dos coeficientes por caminhos é direto a partir das equações acima e a suposição de que as covariâncias entre as variáveis X's e os erros das variáveis Y's são nulos.

Em correspondência ao diagrama de caminhos, associa-se a cada seta unidirecional um coeficiente de trajetória e a cada seta bidirecional um coeficiente de correlação.

A cada diagrama de trajetórias ou caminhos associamos um conjunto de equações de regressão linear, onde cada variável efeito será uma variável dependente no modelo de regressão. 
Somente para exemplificar, note-se que:

$$
r_{x_{1} y_{1}}=n^{-1} \sum_{i=1}^{I} X_{1 i} Y_{1 i}
$$

que, por substituição de uma das equações acima, fica:

$$
\begin{aligned}
& r_{x_{1} y_{1}}=n^{-1} \sum_{i=1}^{n} X_{1 i}\left(\wp_{y_{1} x_{1}} X_{1 i}+\wp_{y_{1} x_{2}} X_{2 i}+e_{y_{1}}\right) \\
& r_{x_{1} y_{1}}=\wp_{y_{1} x_{1}}+\wp_{y_{1} x_{2}} r_{x_{1} x_{2}}
\end{aligned}
$$

uma vez que:

$$
\begin{aligned}
& \sum_{i=1}^{n} X_{1 i} X_{1 i} / n=\sum_{i=1}^{n} X_{1 i}^{2} / n=1 \\
& \sum_{i=1}^{n} X_{1 i} X_{2 i} / n=r_{x_{1} x_{2}} \\
& \sum_{i=1}^{n} X_{1 i} e_{y_{1}}=0
\end{aligned}
$$

A equação (28) envolve dois parâmetros desconhecidos $\wp_{y_{1} x_{1}}$ e $\wp_{y_{1} x_{2}}$, cuja solução é possível, construindo-se uma outra equação com os mesmos parâmetros desconhecidos. Considera-se,

$$
\begin{aligned}
& r_{x_{2} y_{1}}=n^{-1} \sum_{i=1}^{n} X_{2 i} Y_{1 i} \\
& =n^{-1} \sum_{i=1}^{n} X_{2 i}\left(\wp_{y_{1} x_{1}} X_{1 i}+\wp_{y_{1} x_{2}} X_{2 i}+e_{y_{1}}\right) \\
& r_{x_{2} y_{1}}=\wp_{y_{1} x_{1}} r_{x_{1} x_{2}}+\wp_{y_{1} x_{2}}
\end{aligned}
$$

com

$$
\begin{aligned}
& \sum_{i=1}^{n} X_{2 i} X_{2 i} / n=\sum_{i=1}^{n} X_{2 i}^{2} / n=1 \\
& \sum_{i=1}^{n} X_{1 i} X_{2 i} / n=r_{x_{1} x_{2}}
\end{aligned}
$$




$$
\sum_{i=1}^{n} X_{2 i} e_{y_{1}}=0
$$

Logo, resolvendo-se as equações (28) e (30) e usando os supostos (29) e (31), obtêmse os coeficientes por caminhos de $X_{1}$ e $X_{2}$ a $Y_{1}$.

De maneira similar, para $Y_{2}$ pode-se montar um sistema de três equações ou, alternativamente obter as estimativas dos coeficientes de caminhos fazendo a regressão de $Y_{2}$ em relação a $Y_{1}, X_{1}$ e $X_{2}$.

Em um sistema recursivo, o coeficiente de caminhos de "e" e uma variável endógena $Y_{i}$, é igual à raiz quadrada de 1 menos o quadrado do coeficiente de correlação múltipla entre $Y_{i}$ e todas as outras variáveis do modelo, endógenas ou exógenas que a afetem. Assim, segundo o esquema causal da Figura 3, tem-se:

$$
\begin{aligned}
& \wp_{e_{y_{1}}}=\left(1-R_{y_{1} \cdot x_{1} x_{2}}^{2}\right)^{1 / 2} \\
& \wp_{y_{2}}=\left(1-R_{y_{2} \cdot x_{1} x_{2}}^{2}\right)^{1 / 2} .
\end{aligned}
$$

A estimação dos coeficientes por caminhos, torna-se equivalente à estimação das ponderações da regressão padronizada. Mas, é importante ressaltar a diferença entre estes dois tipos de análises. Em análise de regressão, considera-se a regressão da variável dependente sobre todas as variáveis exploratórias em consideração. Por outro lado, em análise por caminhos, usualmente pode estar envolvida mais de uma regressão e a análise consiste em vários estágios, onde em cada um, faz-se uma regressão da variável que é tomada como efeito em relação às suas causas. Por exemplo, o sistema causal da Figura 3 requer duas análises de regressão separadas, para que todos os coeficientes por caminhos sejam estimados: uma regressão de $Y_{1}$ sobre $X_{1}$ e $X_{2}$ e uma outra regressão de $Y_{2}$ sobre $Y_{1}, X_{1}$ e $X_{2}$.

Entretanto, as análises não causais ou de associação entre um conjunto de variáveis são feitas através de análise por caminhos, usando as propriedades e as relações entre os diferentes tipos de correlações (Anderson, 1984). Assim, consegue-se decompor a estrutura de correlações simples das variáveis envolvidas em seus efeitos diretos e indiretos. 
A seguir serão apresentados resultados importantes de análise de trajetória para variáveis numéricas através do modelo de regressão múltipla, ou seja, um sistema com uma única equação recursiva, uma vez que a mesma metodologia é aplicada a cada uma das equações recursivas do sistema considerado. Assim, estaremos em melhor condição para entender a abordagem e aplicações mais sofisticadas que envolvem, por exemplo, caminhos com variáveis não observáveis ou caminhos considerando-se as relaçōes entre os níveis de variáveis categóricas, que é o objetivo do presente trabalho.

Desse modo, a relação entre os coeficientes de trajetória, $\wp_{i j}$, e os coeficientes de regressão, $\beta_{i j . d}$, é obtida escrevendo-se cada equação de regressão do modelo em duas formas equivalentes, cuja apresentação é dada a seguir.

\subsubsection{Análise de trajetória em modelos de regressão múltipla}

A relação entre os coeficientes de trajetória $\wp_{i j}$ e os coeficientes de regressão $\beta_{i j . d}$, onde $d=(1,2, \ldots, i-1, i+1, \ldots, j-1, j+1, \ldots, m, m+1) ; i=$ $1,2, \ldots, m+1 ; j=1,2, \ldots, i-1, i+1, \ldots, m+1 ; i \neq j$, é obtida escrevendo-se cada equação de regressão do modelo em duas formas equivalentes. Assim, para o gráfico da Figura 4; o modelo de regressão linear múltipla associado é:

$$
X_{i}=\beta_{i 1 \bullet 23 \ldots m+1} X_{1}+\beta_{i 2 \bullet 13 \ldots m+1} X_{2}+\ldots .+\beta_{i, m+1 \bullet 123 \ldots m} X_{m+1}+\epsilon_{i}
$$

onde: os $\beta_{i j . d}$ são os coeficientes de regressão. Ou na forma matricial o modelo fica:

$$
\vec{X}_{i}=\mathcal{X} \vec{\beta}+\vec{\epsilon}
$$

onde

$$
\vec{X}_{i}=\left(\begin{array}{c}
x_{i 1} \\
x_{i 2} \\
\vdots \\
x_{i n}
\end{array}\right),
$$


FIGURA 4: Diagrama de trajetória para o modelo de regressão

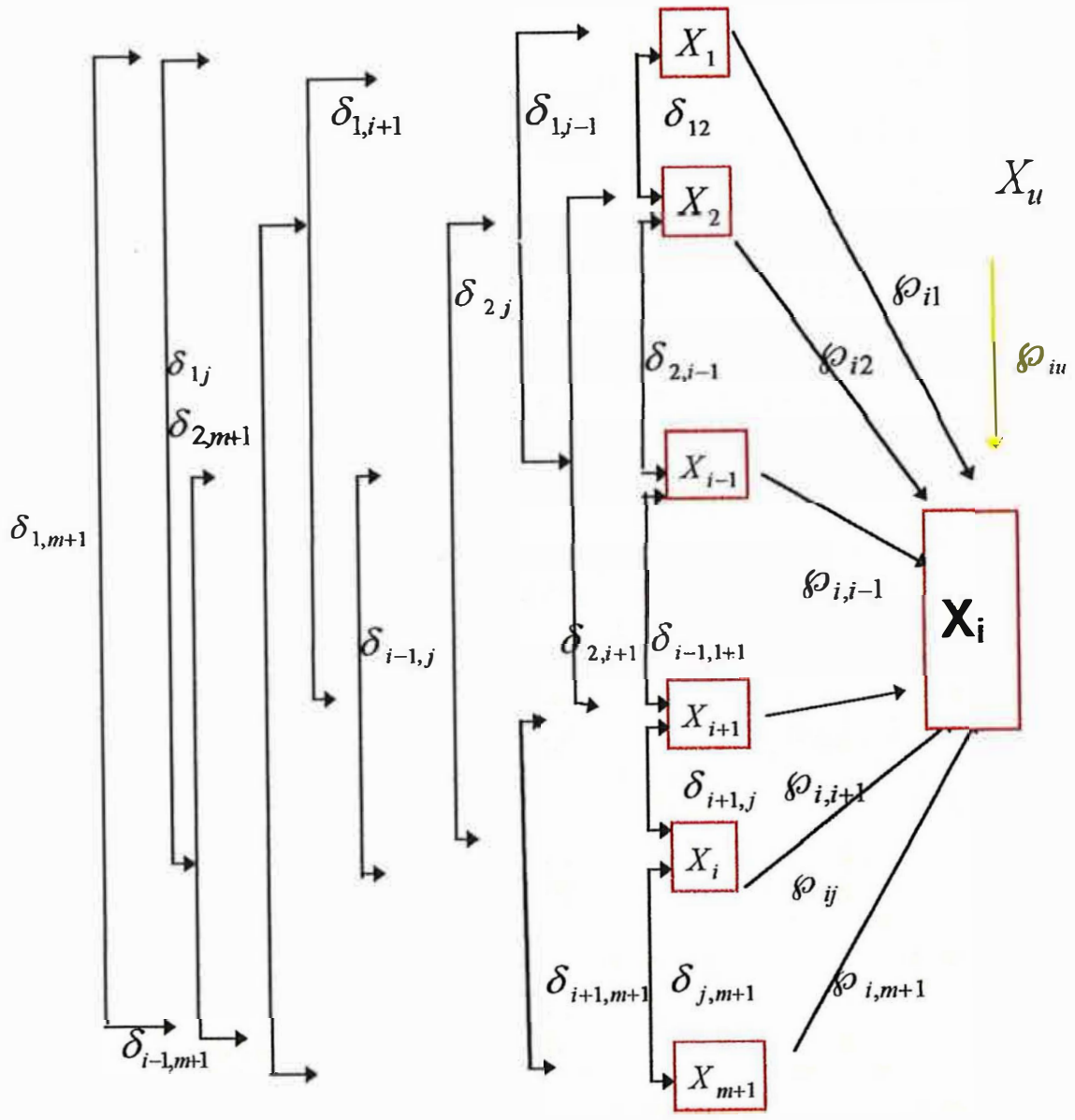

$$
\mathcal{X}=\left(\begin{array}{cccccc}
x_{11} & \ldots & x_{i-1,1} & x_{i+1,1} & \ldots & x_{m+1,1} \\
x_{12} & \ldots & x_{i-1,2} & x_{i+1,2} & \ldots & x_{m+1,2} \\
\vdots & \vdots & \vdots & \vdots & \vdots & \vdots \\
x_{1 n} & \ldots & x_{i-1, n} & x_{i+1, n} & \ldots & x_{m+1, n}
\end{array}\right)
$$




$$
\begin{gathered}
\vec{\epsilon}=\left(\begin{array}{c}
\epsilon_{i 1} \\
\epsilon_{i 2} \\
\vdots \\
\epsilon_{i n}
\end{array}\right) \\
\vec{\beta}=\left(\begin{array}{c}
\beta_{i 1 \bullet 234 \ldots m+1} \\
\beta_{i 2 \bullet 134 \ldots m+1} \\
\vdots \\
\beta_{i m+1 \bullet 12 \ldots m}
\end{array}\right)
\end{gathered}
$$

com $i=(1,2, . ., m+1)$ e $n$ é o número de observações. As dimensões das matrices anteriores são: $\mathrm{nx1}, \mathrm{nxm}, \mathrm{nx} 1 \mathrm{e} \mathrm{mx} 1$ respectivamente.

A partir da equação (32) definem-se

os Coeficientes de Trajetória

$$
\wp_{i j}=\beta_{i j \bullet 123 \ldots(i-1)(i+1) \ldots(j-1)(j+1) \ldots m(m+1)}\left(\sigma_{j j} / \sigma_{i i}\right)^{1 / 2}
$$

Por outro lado, à equação de regressão (32) faz-se corresponder o seguinte modelo de trajetória:

$$
Z_{i}=\wp_{i 1} Z_{1}+\wp_{i 2} Z_{2}+\ldots+\wp_{i, m+1} Z_{m+1}+\wp_{i u} Z_{u}
$$

onde

$$
\begin{aligned}
& Z_{i}=\left(X_{i}-\mu_{i}\right) / \sqrt{\sigma_{i i}}, \\
& i=1,2, \ldots, m+1, \\
& \wp_{i u} Z_{u}=\epsilon_{i},
\end{aligned}
$$

$\mu_{i}$ : média da variável $X_{i}$,

$\sigma_{i i}$ : variância da variável $X_{i}$. Na forma matricial o modelo de trajetória fica

$$
\vec{Z}_{i}=\mathcal{Z} \vec{\wp}+\overrightarrow{\boldsymbol{\epsilon}}
$$


onde

$$
\begin{aligned}
& \vec{Z}_{i}=\left(\begin{array}{c}
z_{i 1} \\
z_{i 2} \\
\vdots \\
z_{i n}
\end{array}\right), \\
& \mathcal{Z}=\left(\begin{array}{cccc}
z_{11} & z_{21} & \ldots & z_{m+1,1} \\
z_{12} & z_{22} & \ldots & z_{m+1,2} \\
\vdots & \vdots & \vdots & \vdots \\
z_{1 n} & z_{2 n} & \ldots & z_{m+1, n}
\end{array}\right),
\end{aligned}
$$

$$
\vec{\wp}=\left(\begin{array}{c}
\wp_{i 1} \\
\wp_{i 2} \\
\vdots \\
\wp_{i, i-1} \\
\wp_{i, i+1} \\
\vdots \\
\wp_{i, m+1}
\end{array}\right),
$$

$$
\overrightarrow{\boldsymbol{\epsilon}}=\left(\begin{array}{c}
\wp_{i u} Z_{u 1} \\
\wp_{i u} Z_{u 2} \\
\vdots \\
\wp_{i u} Z_{u, n}
\end{array}\right)
$$

$$
=\wp_{i u}\left(\begin{array}{c}
Z_{u 1} \\
Z_{u 2} \\
\vdots \\
Z_{u, n}
\end{array}\right)
$$


os coeficientes de trajetória, $\wp_{i j}$, são os coeficientes de regressão quando os dados estão padronizados segundo (37). As dimensões dos vetores anteriores são: $\mathrm{nx} 1, \mathrm{nxm}, \mathrm{mx} 1 \mathrm{e} \mathrm{nx} 1$ respectivamente.

Os coeficientes de trajetória, $\wp_{i j}$, medem o efeito direto da variável $X_{j}$ sobre a variável $X_{i}, i \neq j$. Considerando-se:

$$
\wp_{i j}^{2}=\beta_{i j . d}^{2}\left(\sigma_{j j} / \sigma_{i i}\right)
$$

pode-se dizer que essa expressão mede a proporção da variância da variável dependente, $X_{i}$, da qual $X_{j}$ é diretamente responsável. Neste sentido pode ser considerado como um coeficiente de determinação.

Todas as suposições envolvidas são conhecidas da teoria geral de modelos lineares.

A interpretação de sistemas causais por meio de análise de trajetórias se torna mais eficiente porque permite decompor a influência de uma variável sobre outra em efeitos diretos e outros efeitos.

Algumas dificuldades podem aparecer na aplicação desta técnica quando o sistema causal não é bem determinado.

Por outro lado, usando-se os resultados conhecidos da relação existente entre os coeficientes de regressão, como os coeficientes de correlação parcial e os coeficientes de correlação múltipla, sob suposição de multinormalidade do vetor $\left(X_{1}, X_{2}, \ldots, X_{i}, \ldots X_{j}, \ldots ., X_{m+1}\right)$, segundo Anderson(1984), tem-se:

$$
E\left(X_{i} / X_{1}, X_{2}, \ldots, X_{i-1}, X_{i+1}, \ldots, X_{m}\right)=\sum_{j=1}^{m} \beta_{i j . d} X_{j}
$$

onde os coeficientes de trajetória $\wp_{i j}$, podem ser escritos usando-se as seguintes relações: 
i) o coeficiente de regressão em função das variâncias e covariâncias condicionais;

$$
\beta_{i j . d}=\frac{\sigma_{i j . d}}{\sigma_{j j . d}}
$$

ii) o coeficiente de correlação parcial em função das covariâncias condicionais;

$$
\begin{aligned}
& \varrho_{i j . d}=\frac{\sigma_{i j . d}}{\left(\sigma_{i i . d} \sigma_{j j . d}\right)^{1 / 2}}, \\
& \varrho_{i j . d}\left(\sigma_{i i . d}\right)^{1 / 2}=\frac{\sigma_{i j . d}}{\left(\sigma_{j j . d}\right)^{1 / 2}},
\end{aligned}
$$

iii) o coeficiente de trajetória em função dos coeficientes de regressão, correlações parciais e covariâncias. Assim,

$$
\begin{aligned}
& \wp_{i j}=\beta_{i j . d}\left(\sigma_{j} / \sigma_{i}\right) \\
& =\varrho_{i j . d} \frac{\sigma_{j}}{\sigma_{i}}\left(\sigma_{i i . d} / \sigma_{j j . d}\right)^{1 / 2}
\end{aligned}
$$

iv) ou seja, os coeficientes de trajetória em função das covariâncias condicionais e não condicionais:

$$
\wp_{i j}=\varrho_{i j . d} \frac{\sigma_{j}}{\sigma_{i}}\left(\sigma_{i i . d} / \sigma_{j j . d}\right)^{1 / 2}
$$

Finalmente, usando-se a relação:

$$
1-R_{i . d}^{2}=\frac{\sigma_{i i . d}}{\sigma_{i i}}
$$

v) os coeficientes por caminhos podem ser escritos também, em função das correlações múltiplas, $R_{i . d}$, e das correlações parciais $\varrho_{i j . d}$ :

$$
\begin{gathered}
\wp_{i j}=\varrho_{i j . d}\left(\left(1-R_{i . d}^{2}\right) /\left(1-R_{j . d}^{2}\right)\right)^{1 / 2} \\
i \neq j, j=1,2, \ldots, i-1, i+1, \ldots, m+1 ; d=(1,2, \ldots, j-1, j+1, \ldots, m, m+1)
\end{gathered}
$$


vi) Em particular para $i=m+1$, tem-se

$$
\wp_{m+1, j}=\varrho_{m+1, j . d}\left(\left(1-R_{m+1 . d}^{2}\right) /\left(1-R_{j . d}^{2}\right)\right)^{1 / 2}
$$

Um aspecto muito importante é o que corresponde à estimação dos coeficientes de trajetória.

Um estimador sugerido para o coeficiente de trajetória segundo Achcar(1976) é dado por:

$$
\hat{\wp}_{i j}=\hat{\beta}_{i j . d} \frac{\hat{\sigma}_{j}}{\hat{\sigma}_{i}}
$$

onde os $\hat{\beta}_{i j . d}$ são elementos do vetor,

$$
\hat{\vec{\beta}}=\left(\mathcal{X}^{\prime} \mathcal{X}\right)^{-1} \mathcal{X}^{\prime} \vec{X}_{i}
$$

e $\hat{\sigma}_{i}=s_{i}$ é o desvio padrão da variável $X_{i}, i=1,2, \ldots, m+1$.

Também para as equações (40) e (41) têm-se os estimadores:

$$
\begin{aligned}
& \hat{\wp}_{i j}=\hat{\varrho}_{i j . d} \sqrt{\frac{\hat{\sigma}_{i . d}^{2}}{\hat{\sigma}_{j . d}^{2}} \frac{\hat{\sigma}_{j}}{\hat{\sigma}_{i}}}, \\
& \hat{\wp}_{i j}=\hat{\varrho}_{i j . d}\left(\frac{1-\hat{R}^{2}{ }_{i . d}}{1-\hat{R}_{j . d}^{2}{ }^{1 / 2}},\right.
\end{aligned}
$$

onde,

$i \neq j ; j=1,2, . ., i-1, i+1, \ldots, m+1$,

$\hat{\varrho}_{i j . d}=r_{i j . d}$ : é o coeficiente de correlação parcial na amostra,

$\hat{\sigma}_{i i . d}=\hat{\sigma}_{i . d}^{2}:$ é a variância condicional na amostra e

$\hat{R}_{i . d}$ : é o coeficiente de correlação múltipla na amostra.

Nota-se que este estimador, $\hat{\wp}_{i j}$, usando-se as equações (42), (43) ou (44) não depende da unidade física na qual a variável $X_{i}$ está medida, sendo esta uma diferença básica com o estimador do coeficiente de regressão.

Pode-se, também, estimar os coeficientes de trajetória $\wp_{i j}$ pelo método de mínimos quadrados, aplicando-o diretamente ao modelo de trajetória (36). 
Em continuação será apresentado o teorema fundamental da análise de trajetória ou de caminhos, para o qual também haverá uma versão no capítulo seguinte, para o caso de tabelas de contingência de dupla entrada.

\section{Teorema fundamental da análise por caminhos}

Seja $\hat{\varrho_{i j}}=r_{i j}$, o coeficiente de correlação amostral de Pearson entre as variáveis $X_{i}$ e $X_{j}$, cuja fórmula em função das variáveis padronizadas $Z_{i}$ é

$$
r_{i j}=\frac{1}{n} \sum_{k=1}^{n} Z_{i k} Z_{j k}
$$

Então, de acordo com as equações de regressão associadas a um diagrama de caminhos, tem-se segundo Wright(1934), Achcar(1976):

$$
r_{i j}=\sum_{q} \hat{\wp}_{i q} r_{q j}
$$

onde, q cobre todas as variáveis associadas às possíveis trajetórias entre as variáveis $X_{i}$ e $X_{j}$.

Demostração: A estimativa do modelo de trajetória (36) pode ser escrita como:

$$
Z_{i}=\hat{\wp}_{i 1} Z_{1}+\hat{\wp}_{i 2} Z_{2}+\ldots .+\hat{\wp}_{i, m+1} Z_{m+1}
$$

Substituindo (47) em (45) tem-se

$$
\begin{aligned}
& r_{i j}=\frac{1}{n} \sum_{k=1}^{n} Z_{i k} Z_{j k} \\
& =\frac{1}{n} \sum_{k=1}^{n}\left(\hat{\wp}_{i 1} Z_{1 k}+\hat{\wp}_{i 2} Z_{2 k}+\ldots+\hat{\wp}_{i, m+1} Z_{m+1, k}\right) Z_{j k} \\
& =\frac{1}{n}\left(\hat{\wp}_{i 1} \sum_{k=1}^{n} Z_{1 k} Z_{j k}+\ldots+\hat{\wp}_{i, m+1} \sum_{k=1}^{n} Z_{m+1, k} Z_{j k}\right) \\
& =\hat{\wp}_{i 1} r_{1 j}+\hat{\wp}_{i 2} r_{2 j}+\ldots+\hat{\wp}_{i, m} r_{m j}+\hat{\wp}_{i, m+1} r_{m+1, j}
\end{aligned}
$$

ou

$$
r_{i j}=\sum_{q} \hat{\wp}_{i q} r_{j q}
$$


onde q cobre todas as variáveis associadas, sejam ou não residuais as possíveis trajetórias entre as variáveis $X_{i}$ e $X_{j}$.

Assim, em um diagrama de trajetórias, o coeficiente de correlação de Pearson entre duas variáveis é obtido tomando-se todos os caminhos possíveis entre as variáveis consideradas e tomando-se os produtos entre os valores numéricos dos estimadores dos coeficientes envolvidos ao longo de cada trajeto.

Um caso importante de (46) é quando $\mathrm{i}=\mathrm{j}$ e é conhecido como a fórmula para a determinação completa de $X_{i}$, ou seja:

$$
\begin{aligned}
& r_{i i}=\sum_{q} \hat{\wp}_{i q}^{2}+2 \sum_{q<q^{\prime}} \hat{\wp}_{i q} r_{q q^{\prime}} \hat{\wp}_{i q^{\prime}} \\
& r_{i i}=\sum_{q} \hat{\wp}_{i q} r_{i q}=1
\end{aligned}
$$

onde q e q' (q'>q) incluem todas as variáveis aleatórias envolvidas, sejam ou não residuais.

A partir do resultado anterior pode-se estimar o coeficiente de trajetória residual, $\hat{\wp}_{i u}$. De $(48)$ e colocando-se em evidência $\left(\hat{\wp}_{i u}\right)^{2}$ tem-se:

$$
\begin{aligned}
& 1=\sum_{q *} \hat{\wp}_{i q *}^{2}+2 \sum_{q<q_{*}^{*}} \hat{\wp}_{i q} r_{q q *} \hat{\wp}_{i q *}+\hat{\wp}_{i u}^{2} \\
& \hat{\wp}_{i u}^{2}=1-\sum_{q, q *} \hat{\wp}_{i q} r_{i q *}
\end{aligned}
$$

onde $\mathrm{q}^{*}$ e $\mathrm{q}$ inclui todas as variáveis não residuais. Também,

$$
\hat{\wp}_{i u}^{2}=1-\hat{R}^{2}{ }_{i \bullet 123 \ldots(i-1)(i+1) \ldots(m+1)} .
$$

A seguir a metodologia de análise de trajetória apresentada aqui será desenvolvida com base na Análise de Dependência (Cordeiro, 1990) para as tabelas de contingência bidimensionais, ou seja, será proposto um modelo de trajetória entre os níveis de uma das categorias da tabela de contingência (considera-se um nível como efeito e os outros níveis como causas) em que os níveis da outra categoria da tabela serão considerados como as observações. 


\section{DESENVOLVIMENTO TEÓRICO DA}

\section{TRANSPOSIÇÃO DA METODOLOGIA DE ANÁLISE DE TRAJETÓRIA PARA DADOS CATEGóRICOS}

\subsection{Introdução}

Apresenta-se aqui uma proposta sobre os principais aspectos da metodologia de análise de trajetórias para dados categóricos, que permite a realização das mesmas análises efetuadas em análise de trajetórias com variáveis aleatórias numéricas.

Sabe-se que em tabelas de contingência os dados são as frequências e para variáveis aleatórias nominais ordenadas ou não, não se pode falar de correlação entre elas. As medidas de associação mais usuais tratam de analisar as relações entre as variáveis concorrentes e não entre seus níveis.

Assim, para verificar-se por exemplo, em uma tabela onde uma das dimensões são as espécies de algum animal e na outra os alimentos, aplicando-se as técnicas convencionais pode-se testar se há ou não evidência de associação entre as duas categorias e, em havendo, ajusta-se algum modelo explicativo da mesma (Fienberg, 1980; Bishop et al, 1975; Agresti, 1984; Andersen, 1991; dentre outros).

Poucas técnicas permitem a verificação de algum tipo de associação entre as linhas ou entre as colunas da tabela (Goodman, 1985), como entre as 
espécies do exemplo, do ponto de vista de suas preferências alimentares. E este é um problema muito importante em áreas biológicas, por exemplo.

Além disto, em problemas dessa natureza, é muito importante verificar se as espécies se atraem ( ou se repelem) por si mesmas ou através de ligações em grupos. Ou seja, estudar-se há evidência de associação entre os níveis das categorias e a possibilidade de ajustar-se um modelo explicativo para um nível através dos outros níveis da mesma categoria. Em muitas situações práticas será necessário se proporem relações causais entre os níveis de uma categoria como se fossem os efeitos dos outros níveis da mesma categoria.

Para solucionar o problema descrito, propõe-se aqui utilizar os parâmetros de dependência-co-dependência de Cordeiro(1990) para o desenvolvimento de uma análise por caminhos segundo Wright (1918); Wermuth (1980); Wermuth \& Lauritzen, (1983).

Mas, se o propósito da análise é fazer inferências, é necessário atribuir modelos probabilísticos para os dados. Neste sentido os principais modelos probabilísticos que podem ser associados às tabelas de contingência são: produto de distribuições de Poisson, distribuição multinomial, produto de distribuições multinomiais, entre outros.

Quando todas as frequências da tabela de contingência são variáveis aleatórias, um modelo geralmente utilizado é aquele que associa uma distribuição de Poisson a cada frequência. Isto é, a frequência de cada casela (variável resposta) refere-se à observação de um evento raro segundo uma certa medida a priori (por hora, por página, por animal). Por hipótese, o número de ocorrências do evento em cada casela é gerado por processos de Poisson independentes. Nesse caso não se tem conhecimento a priori do total de observações a serem obtidas e o modelo global para a tabela é um produto de distribuições de Poisson. As caselas são definidas pela combinação dos níveis das demais variáveis em estudo, consideradas como variáveis explicativas. Consequentemente, as caselas representam as sub-populações de interesse. A distribuição multinomial é um modelo probabilístico adequado para as 
situações onde o total geral da tabela é fixado no planejamento da pesquisa. Considera-se uma única amostra aleatória de tamanho igual ao "total geral" da tabela que foi selecionada de uma população supostamente infinita, com probabilidades constantes (e positivas) para toda unidade populacional com as mesmas características.

Sobre o modelo multinomial as variáveis categóricas envolvidas na análise são todas consideradas como variáveis respostas. Cada unidade amostral é classificada de maneira única em uma das distintas categorias de respostas, indexadas por $\mathrm{k}=1, . . \mathrm{r}$ obtidas da combinação dos níveis dessas variáveis. No caso de mais de uma variável resposta, cada categoria de resposta " $k$ " corresponde a um perfil multivariado de resposta. Este tipo de delineamento é conveniente para verificar-se a hipótese de independência, associação parcial ou associação condicional entre as variáveis envolvidas.

Se o esquema de amostragem adotado em uma determinada pesquisa for tal que fixa a priori alguns totais marginais relativos às combinações de níveis de algumas variáveis, um modelo muito utilizado corresponde à distribuição produto de multinomiais. As variáveis para as quais foram fixadas os totais marginais são consideradas como variáveis explicativas; as combinações de seus níveis definem as subpopulações independentes. As demais variáveis são consideradas como variáveis respostas e as combinações de seus níveis geram as categorias de respostas.

Mesmo que a amostragem tenha sido realizada na forma mais conveniente para aplicação da distribuição multinomial, se os objetivos envolverem a explicação do comportamento de uma variável (ou mais de uma) em função de um conjunto de variáveis explicativas, é possível resolver o problema através do condicionamento em totais marginais de interesse. Essa estratégia de condicionamento tem justificativa baseada no fato de que se as contagens da tabela foram geradas segundo o modelo multinomial, i.e., com o total geral fixado por planejamento, então a distribuição condicional das frequências dado os totais marginais de interesse corresponde a um modelo produto de multinomiais (Bishop et al, 1975; Fienberg, 1980). 
Cabe aqui mencionar que os estimadores de máxima verossimilhança das frequências esperadas nas caselas são geralmente os mesmos, qualquer que seja o modelo probabilístico adotado (Agresti, 1984). Isto deve-se ao fato de que tais estimadores para um determinado modelo satisfazem às restrições impostas por outro.

Os aspectos técnicos sobre os modelos probabilísticos para dados categóricos e sobre condicionamento encontram-se em Bishop et al (1975); Fienberg (1980); Agresti (1984) dentre outros.

Consequentemente, sem perda de generalidade a proposta será desenvolvida considerando-se o modelo multinomial, para tabelas de dupla entrada, isto é, com duas categorias.

\subsection{Desenvolvimento do modelo}

\subsubsection{Considerações gerais}

Reiterando o que foi apresentado em (2.1), sejam A e B duas características de classificação de indivíduos de uma população (suposta infinita), com $A_{1}, \ldots, A_{I}$ e $B_{1}, \ldots, B_{J}$ sendo os níveis de $\mathrm{A}$ e $\mathrm{B}$, respectivamente. Considerando-se A a categoria nas linhas e $\mathrm{B}$ a categoria nas colunas, define-se por:

$$
\mathbf{P}=\left[p_{i j}\right]
$$

a matriz de probabilidades, onde;

$$
p_{i j}=P\left(A_{i} \cap B_{j}\right), \quad 1 \leq i \leq I \quad 1 \leq j \leq J
$$

é a probabilidade de um indivíduo escolhido ao acaso desta população pertencer aos níveis $i$ e $j$ das características A e B respectivamente, sendo $p_{i j}>0$, para todo par $(i, j)$.

Denotando-se as probabilidades marginais de linhas e colunas, res-

pectivas, por

$$
\begin{array}{ll}
p_{i .}=\sum_{j=1}^{J} p_{i j} & \text { para } 1 \leq i \leq I \\
p_{. j}=\sum_{i=1}^{I} p_{i j} & \text { para } 1 \leq j \leq J
\end{array}
$$


então, os vetores linhas,

$$
\mathrm{p}_{r, i}^{\prime}=\left(p_{i 1}, p_{i 2}, \ldots, p_{i J}\right) / p_{i .} \quad 1 \leq i \leq I
$$

e os vetores colunas,

$$
\mathbf{p}_{c, j}^{\prime}=\left(p_{1 j}, p_{2 j}, \ldots, p_{I j}\right) / p_{. J} \quad 1 \leq j \leq J
$$

são distribuições de probabilidade. Também o são o vetor linha marginal,

$$
\mathrm{p}_{r,+}^{\prime}=\left(p_{.1}, p_{.2}, \ldots, p_{. J}\right)
$$

e o vetor coluna marginal,

$$
\mathbf{p}_{c,+}^{\prime}=\left(p_{1 .}, p_{2 .}, \ldots, p_{I .}\right)
$$

Estes vetores são denominados perfis-probabilísticos ou somente perfis.

Considerando-se como $\mathrm{r}=\mathrm{I}+\mathrm{J}+2$ o número de perfis de respostas multivariadas (número total de caselas da tabela obtida por cruzamento das duas variáveis categóricas em estudo) e $n$ o tamanho da amostra, a função de probabilidade para o Modelo Multinomial é dado por:

$$
\mathbf{P}(\mathbf{n} / n)=n ! \prod_{j=1}^{J} \prod_{i=1}^{I} \frac{p_{i j}^{n_{i j}}}{n_{i j} !}
$$

com

$$
\begin{aligned}
\mathbf{n}=\left(n_{11}, n_{12}, \ldots, n_{I J}\right) & \text { onde } n_{i j} \text { é a frequência observada } \\
& \text { correspondente aos níveis i e j das } \\
& \text { variáveis A e } \mathrm{B}, \text { respectivamente. }
\end{aligned}
$$

Admitindo-se todas as suposições anteriores, as mesmas suposições

(1)-(6) de ANADEP e considerando-se os resultados de (7)-(20), tem-se

a matriz delta,

$$
\Delta=\frac{1}{\sqrt{2}}\left(\sqrt{\mathbf{P}}-\sqrt{\mathbf{p}_{c,+} \mathbf{p}_{r,+}^{\prime}}\right)
$$


de ordem IxJ, ou seja

$$
\Delta=\frac{1}{\sqrt{2}}\left(\begin{array}{ccc}
\sqrt{p_{11}}-\sqrt{p_{1 . p_{.1}}} & \cdots & \sqrt{p_{1 J}}-\sqrt{p_{1 . p_{. J}}} \\
\vdots & \vdots & \vdots \\
\sqrt{p_{I 1}}-\sqrt{p_{I . p_{.1}}} & \vdots & \sqrt{p_{I J}}-\sqrt{p_{I . p_{. J}}}
\end{array}\right)
$$

a matriz,

$$
D_{c}=\Delta^{\prime} \Delta=\left(\begin{array}{ccc}
d_{c, 11} & d_{c, 12} & d_{c, 1 J} \\
d_{c, 21} & d_{c, 22} & d_{c, 2 J} \\
\vdots & \vdots & \vdots \\
d_{c, J 1} & d_{c, J 2} & d_{c, J J}
\end{array}\right)
$$

que contém as dependências e co-dependências entre as colunas da tabela, a matriz,

$$
\Upsilon_{c}=\left(\begin{array}{cccc}
1 & \delta_{c, 12} & \ldots & \delta_{c, 1 J} \\
\delta_{c, 21} & 1 & \ldots & \delta_{c, 2 J} \\
\vdots & \vdots & \vdots & \vdots \\
\delta_{c, J 1} & \delta_{c, J 2} & \vdots & 1
\end{array}\right)
$$

que contém os coeficientes de co-dependências entre aquelas colunas, onde

$$
\begin{aligned}
& \delta_{c, j j *}=d_{c, j j *} /\left(d_{c, j j} d_{c, j * j *}\right)^{1 / 2} \\
& \delta_{c, j j}=1
\end{aligned}
$$

e para facilitar a abordagem de análise de trajetórias para dados categóricos, a matriz $\Delta$ será escrita da seguinte maneira:

$$
\Delta=\left(\begin{array}{ccccc}
x_{11} & x_{12} & \ldots & x_{1, J-1} & x_{1 J} \\
x_{21} & x_{22} & \ldots & x_{2, J-1} & x_{2 J} \\
\vdots & \vdots & \vdots & \vdots & \vdots \\
x_{I 1} & x_{I 2} & \ldots & x_{I, J-1} & x_{I J}
\end{array}\right)
$$


onde

$$
x_{i j}=\frac{1}{\sqrt{2}}\left(\sqrt{p_{i j}}-\sqrt{p_{i . p_{. j}}}\right) \quad \text { para } i=1, \ldots, I \text { e } j=1, \ldots, J
$$

Neste estágio, dependendo das relações existentes entre os níveis de uma das categorias da tabela de contingência, pode-se fazer a abordagem mediante dois enfoques.

No primeiro caso, considera-se um nível de uma das categorias da tabela de contingência como uma variável resposta (nível resposta) e os outros níveis como variáveis explicativas (níveis explicativos). Neste caso o objetivo é estudar a influência isolada ou combinada dos níveis explicativos na distribuição do nível resposta.

No segundo caso, o interesse está dirigido ao estudo da associação entre os diversos níveis de uma das categorias da tabela de contingência, i.e., para a verificação da existência de algum grau de dependência entre tais níveis. Neste caso todos os níveis da categoria são consideradas como variáveis respostas (níveis respostas).

No contexto descrito, o desenvolvimento de análise de trajetória para tabelas de contingência será apresentado, utilizando-se as relações funcionais (item (3.2.2)) ou levando em conta apenas as relações de associação entre os níveis de uma das categorias da tabela (item (3.2.3)).

O desenvolvimento de análise de trajetórias para dados categóricos será feito levando-se em consideração apenas os resultados das matrizes anteriores para o caso das colunas da tabela de contingência, pois o processo é o mesmo para o caso das linhas, pela substituição das matrizes correspondentes pelas matrizes das linhas. 


\subsubsection{Análise de trajetória através de um modelo de "regressão"}

Para a aplicação de análise de trajetória com variáveis numéricas, inicialmente considera-se um diagrama de trajetória associado as variáveis do problema, (Wright, 1918; Achcar, 1976; Dillon \& Goldstein, 1984; dentre outros).

Nesta proposta, o diagrama de trajetória está associado aos níveis de uma das categorias da tabela de contingência, as colunas.

Emulando a metodologia já bem conhecida para variáveis numéricas e com a finalidade de facilitar a apresentação, considera-se o gráfico de trajetória da Figura 5 , onde os $B_{j}$ são os níveis de $B, j=1, \ldots, J$; que ficam quantificados através das colunas, $X_{j}$, da matriz delta, $\Delta$.

Da mesma maneira que no capítulo anterior, denotar-se-ão os coeficientes de trajetória como $\wp_{i j}$, onde o índice $i$ está associado ao nível efeito e o índice $j$ está associado ao nível causa.

Em correspondência ao diagrama de trajetória, associa-se a cada seta unidirecional um coeficiente de trajetória e a cada seta bidirecional um coeficiente de codependência.

Também a cada diagrama de trajetória associa-se um conjunto de equações às quais chamaremos equações de regressão, onde cada nível efeito será um nível dependente (dos outros níveis) no modelo de regressão proposto.

A relação entre os coeficientes de trajetória, $\wp_{J j}$, e os coeficientes de regressão, $\beta_{J j . l}$, onde $l=(1,2, \ldots, j-1, j+1, \ldots, J-1)$ é obtido escrevendo-se cada equação de regressão do modelo em duas formas equivalentes.

Assim, para o gráfico da Figura 5 associa-se o seguinte modelo de regressão múltipla 
FIGURA 5: Gráfico de trajetória para o modelo proposto

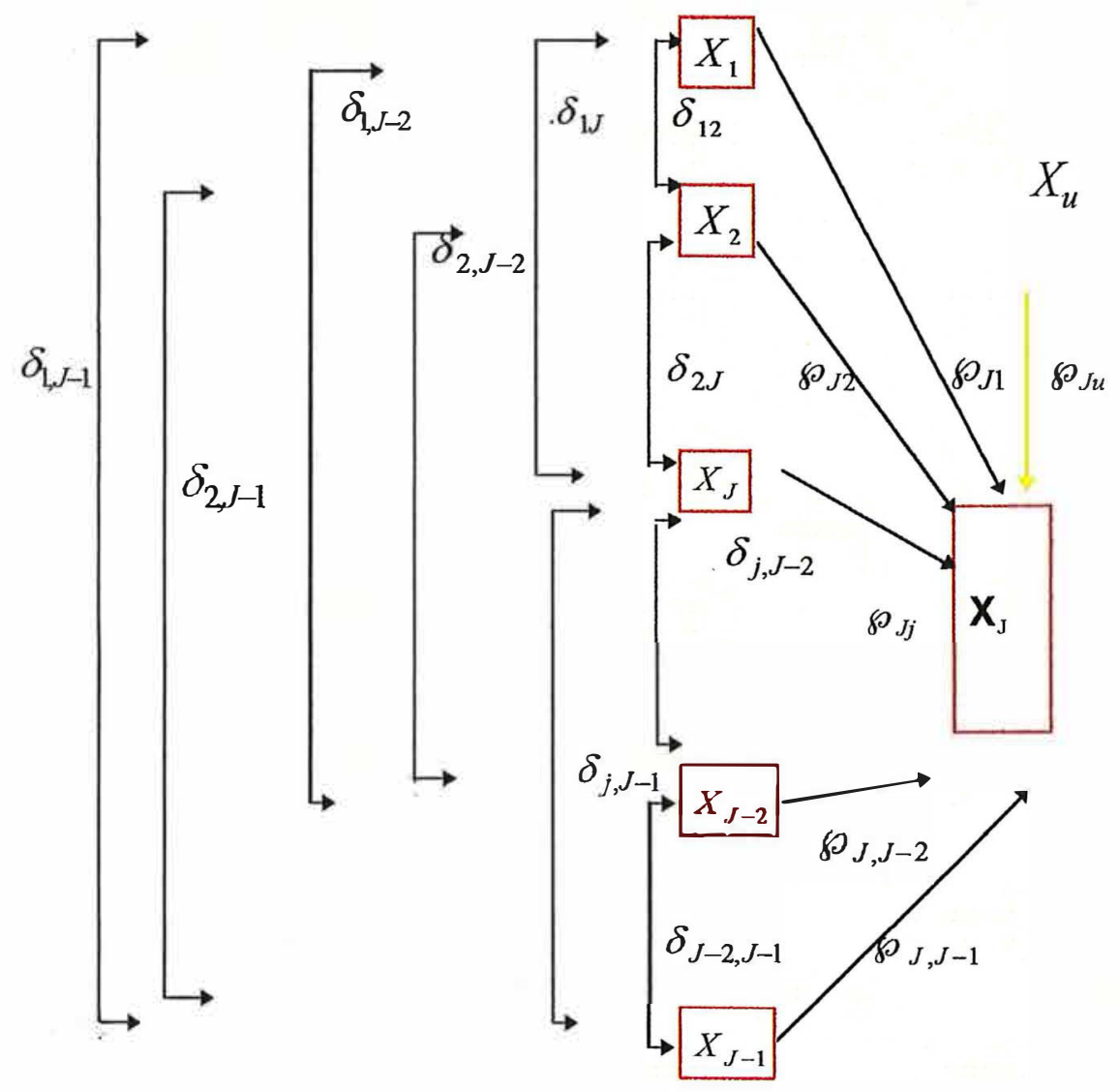

$$
X_{i J}=\beta_{J 1 \bullet 23 \ldots J-1} X_{i 1}+\beta_{J 2 \bullet 13 \ldots J-1} X_{i 2}+\ldots .+\beta_{J, J-1 \bullet 123 \ldots J-2} X_{i, J-1}+\epsilon_{i}
$$

onde:

$$
\begin{array}{cc}
\beta_{J j . l} & \text { são os coeficientes de regressão, } \\
\mathrm{i}=1, \ldots, \mathrm{I} & \mathrm{j}=1, \ldots, \mathrm{J} \\
X_{i j} & \text { valores da j-ésima coluna e i-ésima linha da matriz } \Delta ; \\
\mathrm{I} & \text { é o número de níveis da outra categoria da tabela. }
\end{array}
$$


$\mathrm{Na}$ forma matricial o modelo fica:

$$
\vec{X}_{J}=\mathcal{X} \vec{\beta}+\vec{\epsilon}
$$

onde

$$
\vec{X}_{J}=\left(\begin{array}{c}
x_{1 J} \\
x_{2 J} \\
\vdots \\
x_{I J}
\end{array}\right)
$$

é a J-ésima coluna da matriz $\Delta$, de ordem Ix1,

$$
\mathcal{X}=\left(\begin{array}{cccc}
x_{11} & x_{12} & \ldots & x_{1, J-1} \\
x_{21} & x_{22} & \ldots & x_{2, J-1} \\
\vdots & \vdots & \vdots & \vdots \\
x_{I 1} & x_{I 2} & \ldots & x_{I, J-1}
\end{array}\right)
$$

é a matriz com as colunas restantes da matriz $\Delta$,

$$
\vec{\epsilon}=\left(\begin{array}{c}
\epsilon_{1} \\
\epsilon_{2} \\
\vdots \\
\epsilon_{I}
\end{array}\right)
$$

é o vetor de erros

$$
\vec{\beta}=\left(\begin{array}{c}
\beta_{J 1 \bullet 234 \ldots J-1} \\
\beta_{J 2 \bullet 134 \ldots J-1} \\
\vdots \\
\beta_{J, J-1 \bullet 12 . . J-2}
\end{array}\right),
$$

é o vetor dos coeficientes de regressão a serem estimados. 
Logo, à semelhança da relação (35) define-se o coeficiente de trajetória entre os níveis $J$ e $j$ das colunas da tabela de contingência:

$$
\wp_{J j}=\beta_{J j \bullet 12 \ldots(j-1)(j+1) \ldots(J-1)}\left(d_{c, j j} / d_{c, J J}\right)^{1 / 2} .
$$

Associados ao modelo (49) existem pelo menos dois problemas, quais sejam

i) a estimação dos parâmetros $\beta_{J j . l}$, e portanto, dos $\wp_{J j} \mathrm{e}$

ii) testes de hipóteses para os parâmetros.

Por outro lado, ao modelo de regressão (49) faz-se corresponder o seguinte modelo de trajetória

$$
Z_{i J}=\wp_{J 1} Z_{i 1}+\wp_{J 2} Z_{i 2}+\ldots+\wp_{J, J-1} Z_{i, J-1}+\wp_{J u} Z_{i u}
$$

onde

$X_{j}$ : é a j ésima coluna da matriz $\Delta$ não padronizada,

$$
Z_{j}=\frac{X_{j}}{\sqrt{d_{c, j j}}}
$$

é a j ésima coluna da matriz $\Delta$ padronizada, $j=1,2, \ldots, J \quad i=1,2, \ldots, I$,

$$
d_{c, j j}
$$

é a dependência da j-ésima coluna da tabela de contingência, e

$$
\wp_{J u} Z_{u i}=\epsilon_{i}
$$

$\mathrm{Na}$ forma matricial padronizada, o modelo (52) fica

$$
\overrightarrow{Z_{J}=\mathcal{Z}} \vec{\wp}+\vec{\epsilon}
$$


onde

$$
\begin{aligned}
& \vec{Z}_{J}=\left(\begin{array}{c}
z_{J 1} \\
z_{J 2} \\
\vdots \\
z_{J I},
\end{array}\right) \\
& \mathcal{Z}=\left(\begin{array}{cccc}
z_{11} & z_{12} & \ldots & z_{1, J-1} \\
z_{21} & z_{22} & \ldots & z_{2, J-1} \\
\vdots & \vdots & \vdots & \vdots \\
z_{I 1} & z_{I 2} & \ldots & z_{I, J-1}
\end{array}\right) \\
& \vec{\wp}=\left(\begin{array}{c}
\wp_{J 1} \\
\wp_{J 2} \\
\vdots \\
\wp_{J, J-1}
\end{array}\right) \\
& \overrightarrow{\epsilon_{i}}=\left(\begin{array}{c}
\wp_{J u} Z_{u 1} \\
\wp_{J u} Z_{u 2} \\
\vdots \\
\wp_{J u} Z_{u, I}
\end{array}\right)
\end{aligned}
$$

$\mathrm{O}$ coeficiente de trajetória $\wp_{J j}$, mede o efeito direto do j-ésimo nível da categoria das colunas (B), no J-ésimo nível da mesma categoria, levando-se em conta todos os níveis da categoria das linhas (A) da tabela de contingêneia.

Observa-se que os coeficientes de trajetória, $\wp_{J j}$, estão em função dos coeficientes de regressão $\beta_{J j \bullet 12 \ldots(J-1)}$ e das dependências $d_{c, J J} . \mathbf{e} d_{c, j j}$.

Também, no modelo (52) têm-se que estimar os parâmetros, que neste caso são os coeficientes de trajetória e após, fazer os correspondentes testes de hipóteses. 


\subsubsection{Todos os niveis $\cdot$ da $\cdot$ categoria são níveis respostas}

Uma abordagem alternativa é feita em situações onde existem relações de associação entre os níveis de uma das categorias da tabela de contingência.

Neste caso, a partir da matriz de dependências e codependências, $D_{c}$, são necessárias algumas definições tais como:

i) coeficientes de co-dependência parcial

ii) coeficientes de dependência múltipla

emulando-se o que já se sabe para variáveis contínuas a partir da matriz de covariâncias, e a partir delas propondo-se relações ou equações que relacionem os coeficientes definidos e os coeficientes de trajetória.

Mais uma vez, a metodologia será desenvolvida só para os níveis das colunas, dado que para as linhas o processo é similar.

\section{Coeficiente de Co-dependência Múltipla}

Considerando uma nova apresentação da matriz de dependências e codependências, $D_{c}$, para as colunas da tabela de contingência,

$$
D_{c}=\left(\begin{array}{cccccc}
d_{c, J J} & d_{c, J 1} & \ldots & d_{c, J j} & \ldots & d_{c ; J, J-1} \\
d_{c, 1 J} & d_{c, 11} & \ldots & d_{c, 1 j} & \ldots & d_{c, 1 J-1} \\
\vdots & \vdots & \vdots & \vdots & \vdots & \vdots \\
d_{c, j J} & d_{c ; j 1} & \ldots & d_{c ; j j} & \ldots & d_{c ; j, J-1} \\
\vdots & \vdots & \vdots & \vdots & \vdots & \vdots \\
d_{c ; J-1, J} & d_{c ; J-1,1} & \ldots & d_{c ; J-1, j} & \ldots & d_{c ; J-1, J-1}
\end{array}\right)
$$

cuja primeira coluna contém as dependências-co-dependências do J-ésimo nível $B_{J}$ com ele mesmo e com os demais níveis, de maneira que a partição resultante é:

$$
D_{c}=\left(\begin{array}{c|cc}
d_{c, J J} & \mid & d_{(J)} \\
------- & ------ \\
d_{(J)}^{\prime} & \mid & D_{-J}
\end{array}\right)
$$


onde:

$d_{c, J J}$ é a dependência da J-ésima coluna,

$d_{(J)}$ contém as co-dependências da J-ésima coluna com as colunas restantes, e

$$
D_{-J}=\left(\begin{array}{cccc}
d_{c, 11} & d_{c, 12} & \ldots & d_{c ; 1, J-1} \\
d_{c, 21} & d_{c, 22} & \ldots & d_{c ; 2, J-1} \\
\vdots & \vdots & \vdots & \vdots \\
d_{c ; J-1,1} & d_{c ; J-1,2} & \ldots & d_{c ; J-1, J-1}
\end{array}\right)
$$

é a matriz com as dependências e co-dependências entre as colunas $(1,2, . ., J-1)$.

Com estas anotações, define-se o coeficiente de co-dependência múltipla, necessário para o que se segue.

Definição 3 : Levando em conta as relações (55) - (57) o coeficiente de co-dependência múltipla do nível $B_{J}$ dados os níveis $\left(B_{1}, B_{2}, \ldots, B_{J-1}\right)$ é definido por :

$$
R_{J \bullet 123 \ldots(J-1)}=\sqrt{\frac{d_{(J)} D_{-J}^{-1} d_{(J)}^{\prime}}{d_{c, J J}}}
$$

que pode ser generalizado de maneira a ter-se o coeficiente de co-dependência múltipla da coluna $B_{\boldsymbol{j}}$.qualquer, com as restantes

$$
R_{j \bullet 123 \ldots(j-1)(j+1) \ldots(J-1) J}=\left(\frac{d_{(j)} D_{-j}^{-1} d_{(j)}^{\prime}}{d_{e, j j}}\right)^{1 / 2}
$$

onde, na partição, tem-se :

$d_{(j)}=\left(d_{c, j 1}, d_{c, j 2}, \ldots, d_{c ; j j-1}, d_{c ; j j+1}, \ldots, d_{c, j J}\right)$,

$D_{-j}$ : é a matriz $D_{c}$ sem as j-ésimas linha e coluna,

$d_{c, j j}:$ dependência da j-ésima coluna. 


\section{Coeficiente de Co-dependência Parcial}

Sem perda de generalidade, e à guisa de exemplo, definem-se as codependências condicionais entre os níveis $B_{J}$ e $B_{1}$, dados os níveis restantes. Logo será feita a generalização para dois níveis quaisquer, dados os níveis restantes.

Considere-se a seguinte partição da matriz $D_{c}$ :

$$
D_{c}=\left(\begin{array}{ll}
D_{11} & D_{12} \\
D_{21} & D_{22}
\end{array}\right)
$$

onde:

$$
D_{11}=\left(\begin{array}{ll}
d_{c, J J} & d_{c, J 1} \\
d_{c, 1 J} & d_{c, 11}
\end{array}\right)
$$

é a matriz de dependências e co-dependências das colunas $B_{J}$ e $B_{1}$;

$$
D_{12}=\left(\begin{array}{cccc}
d_{c, J 2} & d_{c, J 3} & \ldots & d_{c_{2} J, J-1} \\
d_{c, 12} & d_{c, 13} & \ldots & d_{c ; 1, J-1}
\end{array}\right)
$$

é a matriz de dependências e co-dependências das colunas $B_{J}, B_{1}$ com as restantes;

$$
D_{22}=\left(\begin{array}{cccc}
d_{c, 22 .} & d_{c, 23} & \ldots & d_{c ; 2, J-1} \\
d_{c, 32} & d_{c, 33} & \ldots & d_{c ; 3, J-1} \\
\vdots & \vdots & \vdots & \vdots \\
d_{c ; J-1,2} & d_{c ; J-1,3} & \ldots & d_{c ; J-1, J-1}
\end{array}\right)
$$

é a matriz de dependências e co-dependências de $B_{2}, \ldots, B_{J-1}$.

Com isto é definida a matriz de dependências e co-dependências condicionais

$$
D_{11.2}=D_{11}-D_{12} D_{22}^{-1} D_{12}^{\prime}=\left(\begin{array}{cc}
d_{c ; J J \bullet 234 \ldots J-1} & d_{c ; j 1 \bullet 234 \ldots J-1} \\
d_{c ; 1 J \bullet 234 \ldots J-1} & d_{c ; 11 \bullet 234 \ldots J-1}
\end{array}\right)
$$


entre as colunas $B_{J}, B_{1}$, dadas as colunas $B_{2}, \ldots, B_{\mathcal{J}-1}$.

A partir do resultado anterior define-se o coeficiente de co-dependências parcial entre $B_{J}$ e $B_{1}$, dados $B_{2}, \ldots, B_{J-1}$ por

$$
\begin{aligned}
& \varrho_{1 J \bullet 23 \ldots J-1}=d_{c ; 1 J \bullet 23 \ldots J-1} /\left(d_{e ; 11 \bullet 23 \ldots J-1} d_{c ; J J \bullet 23 \ldots J-1}\right)^{1 / 2} \\
& =\varrho_{J 1 \bullet 23 \ldots J-1}
\end{aligned}
$$

Se na matriz $D_{c}$ se considerar uma partição mais geral como

$$
D_{11}=\left(\begin{array}{cc}
d_{c, j j} & d_{c, j j *} \\
d_{c, j * j} & d_{c, j * j *}
\end{array}\right)
$$

a matriz de dependências e co-dependências entre as colunas $B_{j}$ e $B_{j *}, j \neq j *$;

$$
D_{12}=\left(\begin{array}{cccc}
d_{c, j 1} & d_{c, j 2} & \ldots & d_{c ; j J} \\
d_{c, j * 1} & d_{c, j * 2} & \ldots & d_{c ; j * J}
\end{array}\right)
$$

a matriz de dependências e co-dependências das colunas $B_{j}, B_{j_{*}}$ com as restantes; $D_{22}$ : matriz de dependências e codependências de $\left(B_{1}, B_{2}, \ldots, B_{j-1}, B_{j+1}, \ldots, B_{j *-1}\right.$, $\left.B_{j *+1}, \ldots, B_{J}\right)$, tem-se a matriz de dependências e codependências condicionais das colunas $B_{j}$ e $B_{j *}$, dadas as demais $(J-2)$ colunas representada por:

$$
D_{11.2}=D_{11}-D_{12} D_{22}^{-1} D_{21}=\left(\begin{array}{cc}
d_{c ; j j . l} & d_{c ; j j * . l} \\
d_{c ; j * j . l} & d_{c ; j * j * . l}
\end{array}\right)
$$

onde $l=(1,2, \ldots, j-1, j+1, \ldots j *-1, j *+1, \ldots, J)$.

A partir desses resultados e sem perda de generalidade, é dada a seguinte definição. 
Definição 4 O coeficiente de co-dependência parcial entre os níveis $B_{j}$ e $B_{j}$, dados os outros níveis $B_{1}, B_{2}, \ldots, B_{j-1}, B_{j+1}, \ldots, B_{j *-1}, B_{j *+1}, \ldots, B_{J}$ é

$$
\varrho_{j j * . l}=d_{c ; j j * l .} /\left(d_{c ; j j . l} d_{c ; j * j * l . l}\right)^{1 / 2}
$$

$$
\begin{aligned}
& 1 \leq j, j *<J \\
& l=(1,2, \ldots, j-1, j+1, \ldots, j *-1, j *+1, \ldots, J)
\end{aligned}
$$

Usando os resultados obtidos e emulando a fórmula (51) podem-se escrever os coeficientes de trajetória em função dos resultados obtidos aqui. Assim,

$$
\wp_{J j}=\beta_{J j \bullet 12 \ldots(j-1)(j+1) \ldots(J-1)}\left(\frac{d_{c, j j}}{d_{c, J J}}\right)^{1 / 2}
$$

e usando a relação

$$
\varrho_{J j . l}=\frac{d_{c ; J j . l}}{\left(d_{c ; J J . l} d_{c ; j j . l}\right)^{1 / 2}}
$$

definem-se os coeficientes de trajetória em função dos coeficientes de co-dependência parcial e das dependências condicionais. Assim, a partir de (63) tem-se:

$$
\begin{aligned}
& \wp_{J j}=\beta_{J j . l}\left(d_{c, j j} / d_{c, J J}\right)^{1 / 2} \\
& \wp_{J j}=\varrho_{J j . l}\left(\frac{d_{c ; J J . l}}{d_{c ; j j . l}}\right)^{1 / 2}\left(\frac{d_{c, j j}}{d_{c, J J}}\right)^{1 / 2} .
\end{aligned}
$$

Finalmente, propondo as relações

$$
\begin{aligned}
& 1-R_{J . l}^{2}=\frac{d_{c ; J J . l}}{d_{c, J J}} \\
& 1-R_{j . l}^{2}=\frac{d_{c ; j j . l}}{d_{c, j j}}
\end{aligned}
$$

os coeficientes por caminhos podem também ser escritos, em função das co-dependências múltiplas, $R_{j . l}^{2}$, e das co-dependências parciais $\varrho_{J j . l}$ :

$$
\wp_{J j}=\varrho_{J j . l}\left(\frac{1-R_{J . l}^{2}}{1-R_{j . l}^{2}}\right)^{1 / 2}
$$




$$
\begin{aligned}
& j=1,2, \ldots, J-1 \\
& l=(1,2, \ldots j-1, j+1, \ldots, J-1) .
\end{aligned}
$$

Cabe ressaltar que, sem perda de generalidade, podem ser definidos os coeficientes de trajetória para qualquer outro grafo de caminhos das seguintes maneiras:

$$
\begin{aligned}
\wp_{j * j} & =\beta_{j * j \cdot 12 \ldots(j-1)(j+1) \ldots(j *-1)(j *+1) \ldots(J-1) J}\left(\frac{d_{c, j j}}{d_{c, j * j *}}\right)^{1 / 2} \\
\wp_{j * j} & =\varrho_{j * j . l}\left(\frac{d_{c ; j * j * l .}}{d_{c ; j j . l}}\right)^{1 / 2}\left(\frac{d_{c, j j}}{d_{c, j * j *}}\right)^{1 / 2} \\
\wp_{j * j} & =\varrho_{j * j . l}\left(\frac{1-R_{j * l l}^{2}}{1-R_{j . l}^{2}}\right)^{1 / 2}
\end{aligned}
$$

$1 \leq j, j * \leq J$

$l=(1,2, \ldots, j-1, j+1, \ldots, j *-1, j *+1, \ldots, J-1, J)$,

onde os níveis $j$ são considerados causas e os níveis $j *$ são considerados efeitos. 


\subsection{Estimação}

\subsubsection{Considerações gerais}

Um aspecto muito importante é o que corresponde à estimação dos coeficientes de trajetória. Seja $T=\left[n_{i j}\right] ; 1 \leq i \leq I, 1 \leq j \leq J$ uma tabela de frequências absolutas de classificação de $n=\sum_{i, j} n_{i j}$ indivíduos amostrados de uma população estratificada de acordo com as categorias $A$ e $B, A=\left(A_{1}, \ldots, A_{I}\right)$ e $B=\left(B_{1}, . ., B_{J}\right)$, onde os $n_{i j}$ é o número de indivíduos.amostrados nos níveis $A_{i} \mathrm{e}$ $B_{j}$. Fazendo:

$$
f_{i j}=\frac{n_{i j}}{n} ; f_{i .}=\sum_{j=1}^{J} f_{i j} ; f_{. j}=\sum_{i=1}^{I} f_{i j} ;
$$

os estimadores de máxima verossimilhança dos parâmetros das relações (1) a (6) são obtidos substituindo-se naquelas expressões $p_{i j}$ por $f_{i j} ; p_{i \text {. }}$ por $f_{i .}$ e $p_{. j}$ por $f_{. j}$.

A partir dessas estimativas são construídas as estimativas das matrizes $D_{c}$ e $\Delta$, por meio das substituições correspondentes.

Assim são estimados os coeficientes de regressão e de dependência e propõe-se a seguinte relação para estimar os coeficientes de trajetória da relação (63):

$$
\hat{\wp}_{J j}=\hat{\beta}_{J j . l} \frac{\hat{d}_{c, j}}{\hat{d}_{c, J}}
$$

onde os $\hat{\beta}_{f j . l}$ são elementos do vetor

$$
\hat{\vec{\beta}}=\left(\mathcal{X}^{\prime} \mathcal{X}\right)^{-1} \mathcal{X}^{\prime} \vec{X}_{J} \quad \text { e } \hat{d}_{c, J}=\left(\hat{d}_{c, J, J}\right)^{1 / 2}
$$

Também para as relações $(64),(65),(66),(67)$ e (68) propõem-se os seguintes estimadores:

$$
\hat{\wp}_{J j}=\hat{\varrho}_{J j . l}\left(\frac{\hat{d}_{c ; J J . l}}{\hat{d}_{c ; j j . l}}\right)^{1 / 2}\left(\frac{\hat{d}_{c, j j}}{\hat{d}_{c, J J}}\right)^{1 / 2}
$$




$$
\begin{aligned}
& \hat{\wp}_{J j}=\hat{\varrho}_{J j . l}\left(\frac{1-\hat{R}_{J . l}^{2}}{1-\hat{R}_{j . l}^{2}}\right)^{1 / 2} \\
& \hat{\wp}_{j * j}=\hat{\beta}_{j * j \bullet 12 \ldots(j-1)(j+1) \ldots(j *-1)(j *+1) \ldots(J-1) J} \frac{\hat{d}_{c, j}}{\hat{d}_{e, j *}} \\
& \hat{\wp}_{j * j}=\hat{\beta}_{j * j . l} \frac{\hat{d}_{c, j}}{\hat{d}_{c, j *}} \\
& \hat{\wp}_{j * j}=\hat{\varrho}_{j * j . l} \frac{\hat{d}_{c, j}}{\hat{d}_{c, j *}}\left(\frac{\hat{d}_{c ; j * j * l}}{\hat{d}_{c ; j j . l}}\right)^{1 / 2} \\
& \hat{\wp}_{j j *}=\hat{\varrho}_{j * j . l}\left(\frac{1-\hat{R}_{j * l}^{2}}{1-\hat{R}_{j . l}^{2}}\right)^{1 / 2}
\end{aligned}
$$

$1 \leq j, j * \leq J ; l=1,2, \ldots, j-1, j+1, \ldots, j *-1, j *+1, \ldots, J-1, J$, onde

$\hat{\varrho}_{J j . l}=r_{J j . l}$ : é o estimador do coeficiente de codependência parcial entre os níveis $B_{J}$ e $B_{j}$ da tabela de contingência, dados os outros níveis;

$\hat{d}_{c ; J J . l}=\hat{d}_{c ; J l l}^{2}$ : é o estimador da dependência condicional de $B_{J}$ dados os outros níveis;

$\hat{R}_{J . l}^{2}$ : estimador do coeficiente de co-dependência múltipla, do nível J com os níveis contidos em $l$.

Por outro lado, também podem ser estimados os coeficientes de trajetória $\wp_{i j}$, pelo método de mínimos quadrados aplicado diretamente ao modelo de trajetória de (52).

Recomenda-se o uso da relação (72) quando á relação funcional entre os niveis de uma das categorias da tabela de contingência, entretanto, as fórmulas (73) e (74) podem ser aplicadas nos casos em que o relacionamento entre os níveis da categoria é associacional.

Em seguida será apresentada uma proposta de análise de caminhos através do Teorema Fundamental de Análise de Trajetória ou de Caminhos para dados categóricos. 
3.3.2 Teorema da análise por caminhos em tabelas de contingência de dupla entrada

Para propor o teorema a seguir sirmułou-se a relação (45) para as variáveis numéricas, utîlizando-se os elementos da matriz $\Delta$ padronizada através de sucessivas tentativas com exemplos consagrados na literatura.

Observou-se que os coeficientes calculados através da expressão:

$$
\delta_{j j *}=\frac{1}{I} \sum_{i=1}^{I} Z_{i j} Z_{i j *}
$$

diferenciam-se dos obtidos através de (19) e pode-se verificar que a diferença entre elas é sempre igual a I. Portanto propoe-se o seguinte teorema.

Teorema Fundamental. Seja uma tabela de contingêneia com duas classifieaçães, onde admite-se I níveis em linhas e $\mathrm{J}$ níveis em colunas.

Sejam, ainda, $B_{j}$ e $B_{j *}$ dois níveis das colunas, define-se o coeficiente de codependêneias entre esses níveis por:

$$
\delta_{j j *}=\sum_{i=1}^{I} Z_{i j} Z_{i j *}
$$

onde $Z_{i j}=X_{i j} /\left(d_{c, j j}\right)^{1 / 2}$ é a j-ésima coluna da matriz $\Delta$ padronizada, $\sum_{i=1}^{I} Z_{i j}^{2}=1, \mathrm{e}$

I é o número de níveis da outra categoria da tabela de contingência.

Então, de acordo com as "equaçōes de regressão" associadas ao diagrama de caminhos, o coeficiente de co-dependência amostral pode ser decomposto em função dos coeficientes de trajetória. Assim,

$$
\hat{\delta}_{c, j j *}=\sum_{q} \hat{\wp}_{j q} \hat{\delta}_{e, j * q}=\sum_{q} \hat{\wp}_{j q} \hat{\delta}_{c, q j *}
$$

onde q cobre todos os níveis associados às possíveis trajetórias entre os níveis $B_{j} \mathrm{e}$ $B_{j} *$. 
Demonstração:

A estimativa do modelo de trajetória $(52)$ para $(\mathrm{J}=\mathrm{j})$ pode ser escrita como

$$
Z_{i j}=\hat{\wp}_{j 1} Z_{i 1}+\hat{\wp}_{j 2} Z_{i 2}+\ldots+\hat{\wp}_{j, J} Z_{i, J}+\hat{\wp}_{j u} Z_{i u}
$$

onde $i=1, \ldots, I$.

$$
\begin{aligned}
& \text { Usando a relação anterior e (75) tem-se } \\
& \hat{\delta}_{c, j j *}=\sum_{i=1}^{I} Z_{i j} Z_{i j *} \\
& =\sum_{i=1}^{I}\left(\hat{\wp}_{j 1} Z_{i 1}+\hat{\wp}_{j 2} Z_{i 2}+\ldots+\hat{\wp}_{j, J} Z_{i, J}+\hat{\wp}_{j u} Z_{i u}\right) Z_{i j *} \\
& =\sum_{i=1}^{I}\left(\hat{\wp}_{j 1} Z_{i 1} Z_{i j *}+\hat{\wp}_{j 2} Z_{i 2} Z_{i j *}+\ldots .+\hat{\wp}_{j, J} Z_{i, j} Z_{i, j *}+\hat{\wp}_{j u} Z_{i u} Z_{i j *}\right) \\
& =\hat{\wp}_{j 1} \hat{\delta}_{c, 1 j *}+\hat{\wp}_{j 2} \hat{\delta}_{c, 2 j *}+\ldots .+\hat{\wp}_{j, J} \hat{\delta}_{c, J j *}+\hat{\wp}_{j u} \hat{\delta}_{u j *}
\end{aligned}
$$

ou

$$
\hat{\delta}_{c, j j *}=\sum_{q} \hat{\wp}_{j q} \hat{\delta}_{c, q j *}=\sum_{q} \hat{\wp}_{j q} \hat{\delta}_{c, j * q}
$$

onde $j, j *=1,2, \ldots, J$ e q cobre todos os níveis associados, sejam ou não residuais, ás possíveis trajetórias entre $B_{j}$ e $B_{j} *$.

Assim, num diagrama de trajetória, o coeficiente de codependência entre dois níveis é obtido tomando-se todos os caminhos possíveis entre os níveis considerados e tomando-se os produtos entre os valores das estimativas dos coeficientes envolvidos ao longo de cada caminho.

Um caso importante de (76) é quando $j=j *$ e pode-se provar que

$$
\hat{\delta}_{c, j j}=\sum_{q} \hat{\wp}_{j q}^{2}+2 \sum_{q<q^{\prime}} \hat{\wp}_{j q} \hat{\delta}_{c, q q^{\prime}} \hat{\wp}_{j q^{\prime}}
$$


onde $j=1, \ldots, J$; q e q'incluem todos os os níveis considerados, sejam ou não residuais.

Demonstração

$$
Z_{i j}=\hat{\wp}_{j 1} Z_{i 1}+\hat{\wp}_{j 2} Z_{i 2}+\ldots . .+\hat{\wp}_{j, J} Z_{i, J}+\hat{\wp}_{j u} Z_{i u} \text { com } i=1, \ldots, I
$$

$\hat{\delta}_{c, j j}=\sum_{i=1}^{I} Z_{i j} Z_{i j}$

$=\sum_{i=1}^{I}\left(\hat{\wp}_{j 1} Z_{i 1}+\hat{\wp}_{j 2} Z_{i 2}+\ldots+\hat{\wp}_{j, J} Z_{i, J}+\hat{\wp}_{j u} Z_{i u}\right)\left(\hat{\wp}_{j 1} Z_{i 1}+\hat{\wp}_{j 2} Z_{i 2}+\ldots+\hat{\wp}_{j, J} Z_{i, J}+\hat{\wp}_{j u} Z_{i u}\right)$ assim

$$
\hat{\delta}_{c, j j}=\sum_{q} \hat{\wp}_{j q}^{2}+2 \sum_{q<q^{\prime}} \hat{\wp}_{j q} \hat{\delta}_{c, q q^{\prime}} \hat{\wp}_{j q^{\prime}}
$$

A partir do resultado anterior pode-se estimar o coeficiente de trajetória residual.

Assim, para a Figura 5, usando-se (77) e colocando em evidência $\hat{\wp}_{J u}^{2}$ tem-se:

$$
1=\hat{\delta}_{c, J J}=\sum_{q=1}^{J-1} \hat{\wp}_{J q}^{2}+2 \sum_{q<q^{\prime}} \hat{\wp}_{J q} \hat{\delta}_{q q^{\prime}} \hat{\wp}_{J q^{\prime}}+\hat{\wp}_{J u}^{2}
$$

de onde,

$$
\hat{\wp}_{J u}^{2}=1-\sum_{q} \hat{\wp}_{J q} \hat{\delta}_{c, J q}
$$

ou

$$
\hat{\wp}_{J u}^{2}=1-\hat{R}_{J \bullet 123 \ldots(J-1)}^{2}
$$

que seria a proporção da dependência do nível J que não foi explicada pelos outros níveis .

Se na equação (77) colocamos em evidência $\hat{\wp}_{J u}^{2} \quad$ e logo for multiplicada pela dependência de $B_{j}$ tem-se

$$
d_{c, J J}=d_{c, J J} \sum_{q} \hat{\wp}_{J q}^{2}+d_{c, J J} 2 \sum_{q<q^{\prime}} \hat{\wp}_{J q} \hat{\delta}_{q q^{\prime}} \hat{\wp}_{J q^{\prime}}+d_{c, J J} \hat{\wp}_{J u}^{2}
$$


ou

$$
\begin{aligned}
& \left(\begin{array}{cc}
\text { dependência } & \\
\text { total } & \text { de } \\
B_{J}
\end{array}\right)=\left(\begin{array}{cc}
\text { proporção } & \text { dada } \\
\text { diretamente } & \text { pelos } \\
\text { estimadores } & \text { dos } \\
\text { coeficientes } & \text { de } \\
\text { caminhos } &
\end{array}\right) \\
& +\left(\begin{array}{cc}
\text { proporção } & \text { devida } \\
\text { às } & \text { interdependências } \\
\text { entre } & \text { níveis } \\
\text { regressores } &
\end{array}\right)+\left(\begin{array}{c}
\text { proporção } \\
\text { devida } \\
\text { ao } \\
\text { resíduo }
\end{array}\right)
\end{aligned}
$$

O que foi feito é a decomposição dos coeficientes de codependência $\left(\delta_{c, j j^{*}}\right)$, através dos coeficientes de trajetória $\left(\wp_{j * j}\right)$. Assim, os coeficientes de trajetória podem ser usados para reproduzir os coeficientes de codependência existentes entre os níveis das colunas (idem para linhas) da tabela de contingêneia.

\subsection{Testes de hipótese para os coeficientes de trajetória}

Seguindo a mesma metodologia de análise de regressão para variáveis numéricas podem-se propor os testes de interesse para os parâmetros do modelo de trajetória (52). Emula-se aqui a metodologia proposta por (Dillon e Goldstein, 1984) para variáveis numéricas. Se ela fosse válida para tabelas de contingência de dupla entrada, a metodologia proposta seria a seguinte:

i) Seja p o número de equações de regressão associadas ao diagrama de trajetória,

ii) Seja s o número de restrições impostas ao modelo,

iii) Obter o quadrado do coeficiente de codependência múltipla $\left(\hat{R}_{i . l}^{2}\right)$ associado à i-ésima equação para o modelo sem restrição, $i=1, \ldots, p$, 
iv) Calcular $\hat{R}_{m}^{2}=1-\left(1-\hat{R}_{1 . l}^{2}\right) \ldots\left(1-\hat{R}_{\dot{p} . l}^{2}\right)$

v) Obter os coeficientes de codependência múltipla $\left(\hat{R}_{i . l}^{* 2}\right)$ associados à i-ésima equação do modelo sob a hipótese nula,

vi) Calcular $M=1-\left(1-R_{1 . l}^{* 2}\right) \ldots . .\left(1-R_{p . l}^{* 2}\right)$,

vii) Calcular $W=-(I-s) \ln \left(1-\hat{R}_{m}^{2} / 1-M\right)$.

viii) A estatística $W$, assintoticamente, tem distribuição qui-quadrado com s graus de liberdade

Assim, a estatística $W$ poderia ser usada para testar que alguns coeficientes de trajetória são nulos.

Neste estudo verificou-se a compatibilidade desta proposta de teste com metodologias inseridas nos pacotes estatísticos usuais para ajuste de modelos de regressão, tais como STATGRAPHICS versão 6.0 e o SAS versão 6.07.

A determinação correta de um teste adequado é uma questão em aberto e deve ser explorado com mais-cuidado. Considere-se a presente proposta só como uma primeira aproximação à solução do problema. 


\section{APLICAÇÃO DA METODOLOGIA}

\subsection{Descrição dos dados}

Com o objetivo de apresentar uma aplicação da metodołogia proposta, usou-se os dados analisados por Bélo (Bélo \& Lavaca, 1982) em um estudo de associação entre Drosophyla e Leveduras, quanto à preferência alimentar. Os resultados referem-se a 5340 moscas classificadas segundo 5 espécies de Drosophyla e 12 espécies de leveduras, conforme consta na Tabela 7.

Cabe mencionar que Drosophyla e leveduras talvez se incluam entre os casos mais fascinantes de associação transportador-simbionte, prestando-se, portanto a estudos de coevoluçāo.

Estudaram-se as seguintes espécies de Drosophyla:

i) D. ANANASSAE (A)

ii) D. HYDEI (H)

iii) D. KIKKAWAI (K)

iv) D. MELANOGASTER (M)

v) D. SIMULANS (S).

Consideraram-se os seguintes gêneros de espécies de leveduras:

1. CAB-CKR-CMA-CPT-CTR

2. CÂNDIDA (espécies CA-1 a CA-4) 
TABELA 7: Classificação das moscas segundo leveduras

\begin{tabular}{lrrrrrr}
\hline & \multicolumn{7}{c}{ Espécies de Drosephyla } \\
LEVEDURAS & A & H & K & M & S & Total \\
\hline CAB- CTR & 142 & 74 & 84 & 151 & 232 & 683 \\
CANDIDA & 81 & 99 & 105 & 88 & 117 & 490 \\
L1-L5 & 98 & 54 & 40 & 87 & 98 & 377 \\
HANSENDU & 93 & 92 & 282 & 165 & 205 & 837 \\
L6-L11 & 113 & 108 & 142 & 78 & 300 & 741 \\
PICHIA & 110 & 55 & 173 & 211 & 130 & 679 \\
G.RHODOTH & 143 & 2 & 95 & 21 & 45 & 306 \\
G. SACCHA & 65 & 3 & 41 & 43 & 92 & 244 \\
L12- L14 & 40 & 93 & 56 & 110 & 64 & 363 \\
TORULOP & 46 & 41 & 49 & 102 & 121 & 359 \\
G.TRICOSP & 17 & 22 & 43 & 40 & 42 & 164 \\
L15 & 10 & 14 & 34 & 20 & 19 & 97 \\
\hline TOTAL & 958 & 657 & 1144 & 1116 & 1465 & 5340 \\
\hline
\end{tabular}

3. L1-L5

4. HANSENULA (espécies HA-1 a HA-6)

5. L6- L11

6. PICHIA (espécies PI-1 a PI-4)

7. RHODOTORULA (espéeies RO-1 e RO-2)

8. SACCHAROMYCES (espécies SA-1 e SA-2)

9. L12-L14 
10. TORULOPSIS (espécies TO-1 a TO-4)

11. TRICOSPORON (espécies TR-1 a TR-2)

12. L15.

As espécies foram recodificadas da seguinte forma:

i) Espécie D. Ananassae (A) ; ou 1 ; ou $X_{1}$; ou $Z_{1}$

ii) Espécie D.Hydei (H) ; ou 2; ou $X_{2}$; ou $Z_{2}$

iii) Espécie D. Kikkawai (K) ; ou 3; ou $X_{3}$; ou $Z_{3}$

iv) Espécie Melanogaster (M); ou 4; ou $X_{4}$; ou $Z_{4}$

v) Espécie Sinulans (S) ; ou 5 ; ou $X_{5}$; ou $Z_{5}$

Há interesse em propor um modelo de associação para a espécie Drosophyla e leveduras através dos coeficientes de deperdêtreias e codependências. Logo, pretende-se decompor os coeficientes de codependência em seus efeitos diretos e indiretos. Esse estudo será feito através da análise de trajetória, para o qual é necessário inicialmente considerar-se um diagrama de trajetórias, associado aos níveis das características do problema em questão. A aplicação será feita em duas fases.

I) Na primeira fase é feita a análise de dependência (passo prévio à análise de trajetória) segundo a metodologia apresentada no capítulo 2. Nesta etapa são calculadas as dependências-codependências e os coeficientes de codependências.

II) Na segunda fase é feita a análise de trajetórias com base nos resultados do capítulo anterior. Esta fase também tem duas etapas

1. A estimação dos coeficientes de trajetória.

2. A decomposição dos coeficientes de codependência em seus efeitos diretos (que são os coeficientes de trajetória) e os outros efeitos. 
Nesta fase é proposto um diagrama de trajetórias associado aos níveis da espécie Drosophrylb. Esse diagrama dá ofigem a um sistema de equações de regressão que permite estimar os coeficientes de regressão e atra vés de (69) sã estimados os coeficientes de trajetôria.

Também, esses coeficientes de trajetória podem ser estimados diretamente, com a mesmo esquema de trajetórias, só que os dados da matriz delta, previamente serão padronizados conforme (53).

Cabe ressaltar que esses coeficientes de trajetórias podem ser, também, estimados usando-se as relações existentes entre os coeficientes de regressão, os coeficientes de codependência parciais, coeficientes de dependência condicionais e os coeficientes de dependência múltipla, definidos, segundo (66), (67), (68)

Usando o Teorema Fundamental da análise de trajetórias, são decompostos, os coeficientes de codependências entre dois níveis da espécie Drosophyla, em efeitos diretos e outros efeitos segunda (76).

\subsection{Resultados da análise de dependência}

A partir dos dados da Tabela 7, obtêm-se:

A distribuição das frequêneias relativas, os vetores perfis colunas (para a espécie Drosophyla),$\hat{\mathbf{p}}_{c, j}$, pertencentes ao espaço $R^{I}=R^{12}$ e o perfil marginal coluna.

A seguir apresenta-se para os níveis da espécie Drosaphyla, as Tabelas 8,9 as quais referem-se às dependêtreias-co-tepentêreias e aos coefieientes de co-dependência respectivamente, e a matriz $\Delta$, com dependência tatal (Cordeiro, 1990):

$$
\hat{d}^{2}=(39,024+51,18+36,75+35,89+29,93) 10^{-4}=0,019277 .
$$


TABELA 8: Dependências e Co-dependências da Espécie Drosophyla

\begin{tabular}{r|c|rrrrr}
\hline & Espécies & A & H & K & M & S \\
\hline \multirow{1}{*}{$\hat{D}_{c}=$} & A & 0,003902 & $-0,002909$ & $-0,000662$ & $-0,001659$ & $-0,000384$ \\
& H & $-0,002909$ & 0,005118 & $-0,001111$ & 0,001348 & 0,000385 \\
& K & $-0,000662$ & $-0,001111$ & 0,003675 & $-0,000748$ & $-0,001576$ \\
& $\mathrm{M}$ & $-0,001659$ & 0,001348 & $-0,000748$ & 0,003589 & $-0,000949$ \\
& $\mathrm{~S}$ & $-0,000384$ & 0,000385 & $-0,001576$ & $-0,000949$ & 0,002993 \\
\hline
\end{tabular}

TABELA 9: Coeficientes de Co-dependências entre os níveis da Espécie Drosophyla

\begin{tabular}{r|c|rrrrr}
\hline & Espécies & $\mathrm{A}$ & $\mathrm{H}$ & $\mathrm{K}$ & $\mathrm{M}$ & $\mathrm{S}$ \\
\hline \multirow{4}{*}{$\hat{\Upsilon}=$} & $\mathrm{A}$ & 1 & $-0,650986$ & $-0,174852$ & $-0,443187$ & $-0,112351$ \\
$\mathrm{H}$ & $-0,650986$ & 1 & $-0,255887$ & 0,314579 & 0,098453 \\
& $K$ & $-0,174852$ & $-0,255887$ & 1 & $-0,205883$ & $-0,475150$ \\
$\mathrm{M}$ & $-0,443187$ & 0,314579 & $-0,205883$ & 1 & $-0,289599$ \\
& $\mathrm{~S}$ & $-0,112351$ & 0,098453 & $-0,475150$ & $-0,289599$ & 1 \\
\hline
\end{tabular}

$$
\hat{\Delta}=\left(\begin{array}{rrrrr}
0,008196 & -0,005463 & -0,028363 & 0,003298 & 0,014930 \\
-0,003637 & 0,021147 & 0,000012 & -0,007148 & -0,007525 \\
0,016213 & 0,005205 & -0,025763 & 0,004365 & -0,002617 \\
-0,025258 & -0,005382 & 0,032920 & -0,003683 & -0,008086 \\
-0,008705 & 0,008168 & -0,006610 & -0,034956 & 0,029635 \\
-0,005311 & -0,016680 & 0,010568 & 0,025289 & -0,021740 \\
0,044018 & -0,045688 & 0,015968 & -0,033039 & -0,023748 \\
0,013993 & -0,036258 & -0,008001 & -0,005646 & 0,013644 \\
-0,016888 & -0,028649 & -0,012920 & 0,017206 & -0,019153 \\
-0,012027 & -0,002351 & -0,017125 & 0,013912 & 0,010409 \\
-0,012591 & 0,001921 & 0,006097 & 0,004549 & -0,002196 \\
-0,009766 & 0,002777 & 0,012312 & -0,000293 & -0,007738
\end{array}\right)
$$


De acordo com a interpretação dos coeficientes de co-dependências dado em (19) e com os resultados apresentados na Tabela 9, do ponto de vista das suas preferências pelas leveduras, as espécies Drosophyla são muito diferentes, assim:

A espécie " $\mathrm{H}$ " tende a preferir leveduras que a espécie " $\mathrm{A}$ " não prefere;

A espécie " $M$ " tende a preferir leveduras que a especie " $A$ " não prefere, e vice-versa;

As espécies "H" e "S" estão na esfera $R^{12}$ em posição que formam um ângulo quase reto em relação à raiz quadrada do perfil probabilístico marginal.

Do ponto de vista das preferências pelas leveduras, as espécies de moscas são muito diferentes. Codependências baixas não implicam independência na tabela de contingência.

A dependência total é $\hat{d}^{2}=192,779 \times 10^{-4}$, o que corresponde a um valor qui-quadrado de Pearson $X^{2}=8 n \hat{d}^{2}=823,5$ com 44 graus de liberdade, com valor $p<0,01$, sendo, portanto significativa a associação entre a espécie Drosophyla e Leveduras.

\subsection{Resultados da análise de trajetórias}

\subsubsection{Com dados não padronizados}

Suponha-se que para as espécies de Drosophyla conforme a Tabela 7, é válido o esquema de trajetória da Figura 6.

As equações de regressão correspondentes à Figura 6 são:

$$
\begin{aligned}
& X_{1 i}=\beta_{12.345} X_{2 i}+\beta_{13.245} X_{3 i}+\beta_{14.235} X_{4 i}+\beta_{15.234} X_{5 i}+\epsilon_{1 i} \\
& X_{2 i}=\beta_{21.345} X_{1 i}+\beta_{23.145} X_{3 i}+\beta_{24.135} X_{4 i}+\beta_{25.134} X_{5 i}+\epsilon_{2 i} \\
& X_{3 i}=\beta_{31.245} X_{1 i}+\beta_{32.145} X_{2 i}+\beta_{34.125} X_{4 i}+\beta_{35.124} X_{5 i}+\epsilon_{3 i} \\
& X_{4 i}=\beta_{41.235} X_{1 i}+\beta_{42.135} X_{2 i}+\beta_{43.125} X_{3 i}+\beta_{45.123} X_{5 i}+\epsilon_{4 i}
\end{aligned}
$$


FIGURA 6: Gráfico de trajetória proposto para a espécie Drosophyla

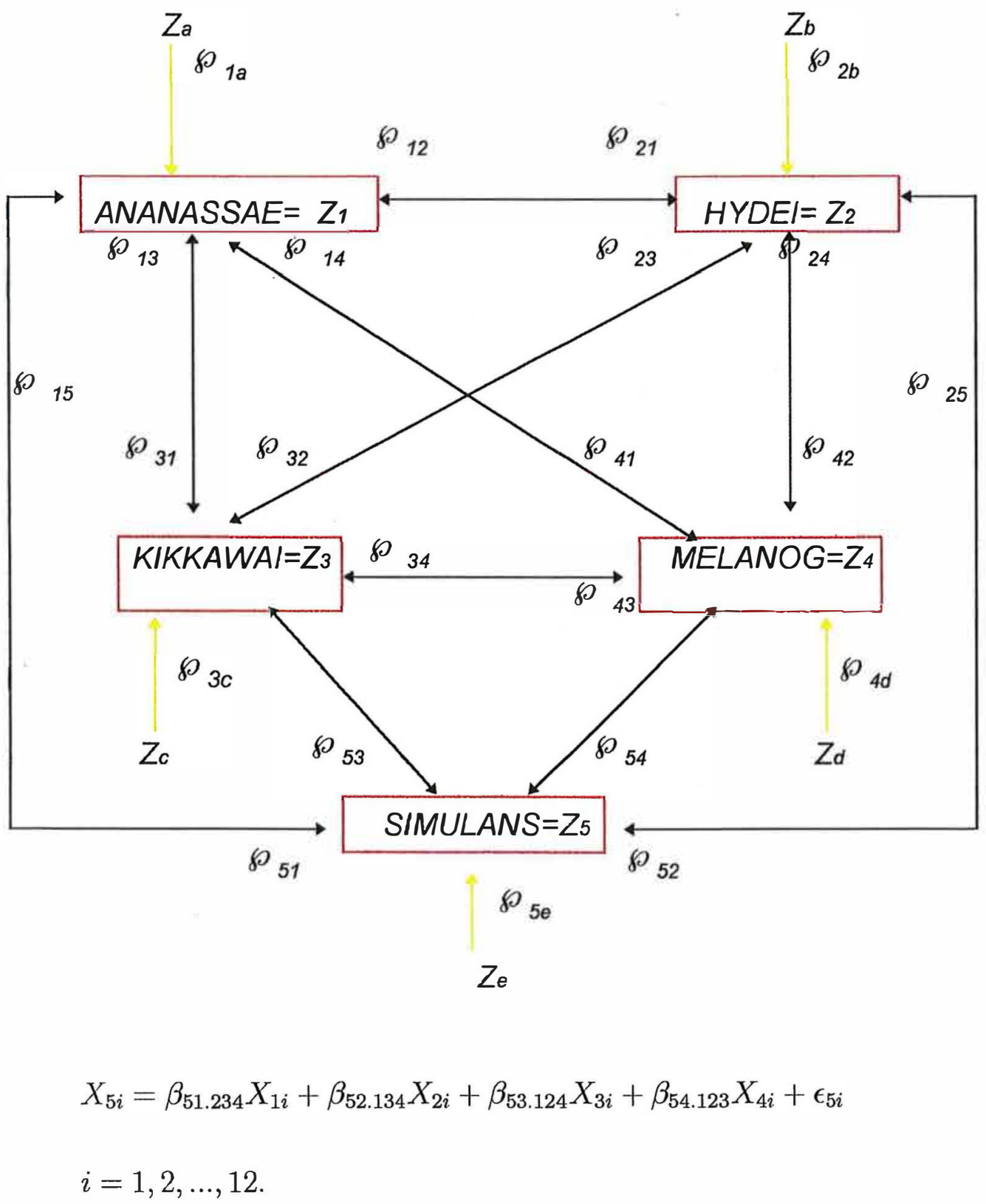

Para cada uma das regressões propostas e usando convenientemente as colunas da matriz $\Delta$, são gerados cinco sistemas de equações, sendo o primeiro 
deles o seguinte sistema:

$$
\Delta^{1}=\left(\begin{array}{r}
0,008196 \\
-0,003637 \\
0,016213 \\
-0,025258 \\
-0,008705 \\
-0,005311 \\
0,044018 \\
0,013993 \\
0,016888 \\
-0,012027 \\
-0,012591 \\
-0,009766
\end{array}\right)=\left(\begin{array}{rrrr}
-0,005463 & -0,028363 & 0,003298 & 0,014930 \\
0,021147 & 0,000012 & -0,007148 & -0,007525 \\
0,005205 & -0,025763 & 0,004365 & -0,002617 \\
-0,005382 & 0,032920 & -0,003683 & -0,008086 \\
0,008168 & -0,006610 & -0,034956 & 0,029635 \\
-0,016680 & 0,010567 & 0,025289 & -0,021741 \\
-0,045688 & 0,015968 & -0,033039 & -0,023748 \\
-0,036258 & -0,008001 & -0,005646 & 0,013644 \\
0,028649 & -0,012920 & 0,017206 & -0,019153 \\
-0,002350 & -0,017125 & 0,013912 & 0,010409 \\
0,001920 & 0,006096 & 0,004549 & -0,002196 \\
0,002777 & 0,012312 & -0,000293 & -0,007738
\end{array}\right)
$$$$
\left(\begin{array}{c}
\beta_{12.345} \\
\beta_{13.245} \\
\beta_{14.235} \\
\beta_{15.234}
\end{array}\right)+\left(\begin{array}{c}
\epsilon_{1} \\
\epsilon_{2} \\
\vdots \\
\vdots \\
\epsilon_{12}
\end{array}\right)
$$

Resolvendo os sistemas de equações gerados, obtêm-se as seguintes estimativas dos " coeficientes de regressão":

$$
\begin{aligned}
& \left(\begin{array}{l}
\hat{\beta}_{12.345} \\
\hat{\beta}_{13.245} \\
\hat{\beta}_{14.235} \\
\hat{\beta}_{15.234}
\end{array}\right)=\left(\begin{array}{l}
-0,525349 \\
-0,726412 \\
-0,582066 \\
-0,627769
\end{array}\right) \\
& \left(\begin{array}{l}
\hat{\beta}_{21.345} \\
\hat{\beta}_{23.145} \\
\hat{\beta}_{24.135} \\
\hat{\beta}_{25.134}
\end{array}\right)=\left(\begin{array}{l}
-1,212013 \\
-0,933867 \\
-0,563311 \\
-0,697147
\end{array}\right)
\end{aligned}
$$




$$
\begin{aligned}
& \left(\begin{array}{l}
\hat{\beta}_{31.245} \\
\hat{\beta}_{32.145} \\
\hat{\beta}_{34.125} \\
\hat{\beta}_{35.124}
\end{array}\right)=\left(\begin{array}{l}
-0,885393 \\
-0,493377 \\
-0,638099 \\
-0,779016
\end{array}\right) \\
& \left(\begin{array}{l}
\hat{\beta}_{41.235} \\
\hat{\beta}_{42.135} \\
\hat{\beta}_{43.125} \\
\hat{\beta}_{45.123}
\end{array}\right)=\left(\begin{array}{l}
-0,949121 \\
-0,398145 \\
-0,853661 \\
-0,837179
\end{array}\right) \\
& \left(\begin{array}{l}
\hat{\beta}_{51.234} \\
\hat{\beta}_{52.134} \\
\hat{\beta}_{53.124} \\
\hat{\beta}_{54.123}
\end{array}\right)=\left(\begin{array}{l}
-0,814877 \\
-0,392245 \\
-0,829631 \\
-0,666439
\end{array}\right)
\end{aligned}
$$

A partir dos resultados anteriores e segundo (72) têm-se as estimativas dos coeficientes de trajetória:

$$
\begin{aligned}
& \hat{\wp}_{12}=\hat{\beta}_{12.345} \sqrt{\frac{\hat{d}_{c, 22}}{\hat{d}_{c, 11}}}=-0,601629 \\
& \hat{\wp}_{13}=\hat{\beta}_{13.245} \sqrt{\frac{\hat{d}_{c, 33}}{\hat{d}_{c, 11}}}=-0,704957 \\
& \hat{\wp}_{14}=\hat{\beta}_{14.235} \sqrt{\frac{\hat{d}_{c, 44}}{\hat{d}_{c, 11}}}=-0,558275 \\
& \hat{\wp}_{15}=\hat{\beta}_{15.234} \sqrt{\frac{\hat{d}_{c, 55}}{\hat{d}_{c, 11}}}=-0,549755 \\
& \hat{\wp}_{21}=\hat{\beta}_{21.345} \sqrt{\frac{\hat{d}_{c, 11}}{\hat{d}_{c, 22}}}=-1,058343 \\
& \hat{\wp}_{23}=\hat{\beta}_{23.145} \sqrt{\frac{\hat{d}_{c, 33}}{\hat{d}_{c, 22}}}=-0,791378
\end{aligned}
$$




$$
\begin{aligned}
& \hat{\wp}_{24}=\hat{\beta}_{24.135} \sqrt{\frac{\hat{d}_{c, 44}}{\hat{d}_{c, 22}}}=-0,471783 \\
& \hat{\wp}_{25}=\hat{\beta}_{25.134} \sqrt{\frac{\hat{d}_{c, 55}}{\hat{d}_{c, 22}}}=-0,533105 \\
& \hat{\wp}_{31}=\hat{\beta}_{31.245} \sqrt{\frac{\hat{d}_{c, 11}}{\hat{d}_{c, 33}}}=-0,912335 \\
& \hat{\wp}_{32}=\hat{\beta}_{32.145} \sqrt{\frac{\hat{d}_{c, 22}}{\hat{d}_{c, 33}}}=-0,582207 \\
& \hat{\wp}_{34}=\hat{\beta}_{34.125} \sqrt{\frac{\hat{d}_{c, 44}}{\hat{d}_{c, 33}}}=-0,630646 \\
& \hat{\wp}_{35}=\hat{\beta}_{35.124} \sqrt{\frac{\hat{d}_{c, 55}}{\hat{d}_{e, 33}}}=-0,702937 \\
& \hat{\wp}_{41}=\hat{\beta}_{41.235} \sqrt{\frac{\hat{d}_{c, 11}}{\hat{d}_{c, 44}}}=-0,98956 \\
& \hat{\wp}_{42}=\hat{\beta}_{42.135} \sqrt{\frac{\hat{d}_{c, 22}}{\hat{d}_{c, 44}}}=-0,475378 \\
& \hat{\wp}_{43}=\hat{\beta}_{43.125} \sqrt{\frac{\hat{d}_{c, 33}}{\hat{d}_{c, 44}}}=-0,863751 \\
& \hat{\wp}_{45}=\hat{\beta}_{45.123} \sqrt{\frac{\hat{d}_{c, 55}}{\hat{d}_{c, 44}}}=-0,764387 \\
& \hat{\wp}_{51}=\hat{\beta}_{51.234} \sqrt{\frac{\hat{d}_{c, 11}}{\hat{d}_{c, 55}}}=-0,930517 \\
& \hat{\wp}_{52}=\hat{\beta}_{52.134} \sqrt{\frac{\hat{d}_{c, 22}}{\hat{d}_{c, 55}}}=-0,512944 \\
& \hat{\wp}_{53}=\hat{\beta}_{53.123} \sqrt{\frac{\hat{d}_{c, 33}}{\hat{d}_{c, 55}}}=-0,919386 \\
& \hat{\wp}_{54}=\hat{\beta}_{54.123} \sqrt{\frac{\hat{d}_{c, 44}}{\hat{d}_{c, 55}}}=-0,729917
\end{aligned}
$$




\subsubsection{Com dados padronizados}

Considerando-se o mesmo esquema de trajetória da Figura 6, mas, levando-se em conta as colnnas da matriz $Z$, que são as colunas da matriz $\Delta$ padronizadas segundo $Z_{j i}=X_{j i} /\left(\sqrt{d_{e, j i j}}\right)$, têm-se as seguintes equações de trajetórias correspondentes a esse gráfico de trajetórias:

$$
\begin{aligned}
& Z_{1 i}=\wp_{12} Z_{2 i}+\wp_{13} Z_{3 i}+\wp_{14} Z_{4 i}+\wp_{15} Z_{5 i}+\wp_{1 a} Z_{a i} \\
& Z_{2 i}=\wp_{21} Z_{1 i}+\wp_{23} Z_{3 i}+\wp_{24} Z_{4 i}+\wp_{25} Z_{5 i}+\wp_{2 a} Z_{b i} \\
& Z_{3 i}=\wp_{31} Z_{1 i}+\wp_{32} Z_{2 i}+\wp_{34} Z_{4 i}+\wp_{35} Z_{5 i}+\wp_{3 e} Z_{c i} \\
& Z_{4 i}=\wp_{41} Z_{1 i}+\wp_{42} Z_{2 i}+\wp_{43} Z_{3 i}+\wp_{45} Z_{5 i}+\wp_{4 d} Z_{d i} \\
& Z_{5 i}=\wp_{51} X_{1 i}+\wp_{52} Z_{2 i}+\wp_{53} Z_{3 i}+\wp_{54} Z_{4 i}+\wp_{5 e} Z_{e i} \\
& i=1,2, \ldots, 12 .
\end{aligned}
$$

Aplicando-se a mesma metodologia descrita no item anterior para cada uma das novas regressões propostas e usando-se convenientemente as colunas da matriz $\Delta$ padronizada $(\mathbf{Z})$,

$$
\mathbf{Z}=\left(\begin{array}{rrrrr}
0,131203 & -0,076365 & -0,467855 & 0,055047 & 0,272926 \\
-0,058217 & 0,295604 & 0,000204 & -0,119297 & -0,137562 \\
0,259534 & 0,072758 & -0,424960 & 0,072849 & -0,047840 \\
-0,404329 & -0,075233 & 0,543025 & -0,061473 & -0,147806 \\
-0,139354 & 0,114175 & -0,109028 & -0,583427 & 0,541718 \\
-0,085010 & -0,233165 & 0,174317 & 0,422085 & -0,397404 \\
0,704644 & -0,638649 & 0,263394 & -0,551416 & -0,434108 \\
0,223998 & -0,506826 & -0,131979 & -0,094238 & 0,249402 \\
-0,270345 & 0,400473 & -0,213118 & 0,287173 & -0,350113 \\
-0,192530 & -0,032849 & -0,282486 & 0,232188 & 0,190293 \\
-0,201536 & 0,026846 & 0,100563 & 0,075928 & -0,040136 \\
-0,156337 & 0,038827 & 0,203091 & -0,004893 & -0,141459
\end{array}\right)
$$


obtêm-se as estimativas correspondentes. Assim têm-se:

$$
\begin{aligned}
& Z_{1 i}=-0,6016 Z_{2 i}-0,7049 Z_{3 i}-0,5583 Z_{4 i}-0,5497 Z_{5 i} \\
& Z_{2 i}=-1,0583 Z_{1 i}-0,7914 Z_{3 i}-0,4718 Z_{4 i}-0,5331 Z_{5 i} \\
& Z_{3 i}=-0,9123 Z_{1 i}-0,5822 Z_{2 i}-0,6306 Z_{4 i}-0,7030 Z_{5 i} \\
& Z_{4 i}=-0,9896 Z_{1 i}-0,4754 Z_{2 i}-0,8638 Z_{3 i}-0,7644 Z_{5 i} \\
& Z_{5 i}=-0,9305 Z_{1 i}-0,5129 Z_{2 i}-0,9194 Z_{3 i}-0,7299 Z_{4 i} \\
& i=1,2, \ldots, 12 .
\end{aligned}
$$

Observa-se que os coeficientes dessas regressões são os mesmos coeficientes de trajetórias já obtidos segundo a proposta inicial.

\subsubsection{Considerando apenas relações de associação}

Neste caso tem-se que considerar as partições adequadas da matriz de dependências e codependências, $D_{c}$, para se obterem as estimativas dos coeficientes de trajetória, segundo (74).

A seguir é descrita detalhadamente a metodologia para se obterem os coeficientes de trajetórias a partir de qualquer espécie até a espécie Simulans (S). Aqui se usou a mesma recodificação anteriọ.

D. ANANASSAE (A); ou 1 ; ou $X_{1}$; ou $Z_{1}$

D. HYDEI (H); ou 2 ; ou $X_{2}$; ou $Z_{2}$

D. KIKKAWAI (K); ou 3 ; ou $X_{3}$; ou $Z_{3}$

D. MELANOGASTER (M); ou 4 ; ou $X_{4}$; ou $Z_{4}$

D. SIMULANS (S); ou 5 ; ou $X_{5}$; ou $Z_{5}$.

e a matriz de dependências e codependências da tabela 8 . 
Cálculo das trajetórias das outras espécies até a espécie $\mathrm{S}=5$

i) Obtenção do caminho a partir da espécie A até a espécie S (de 1 até 5)

1. Calcula-se a co-dependência múltipla da espécie 1 com as espécies (2, $3,4)$. Considera-se apenas a matriz de dependências e co-dependências entre as espécies $(1,2,3,4)$ subdividida da seguinte maneira:

$$
\begin{gathered}
\left(\begin{array}{rrrrr} 
& A & H & K & M \\
A & 0,003902 & -0,002909 & -0,000662 & -0,001659 \\
-- & -- & -- & -- & \\
H & -0,002909 & 0,005118 & -0,001111 & +0,001348 \\
K & -0,000662 & -0,001111 & +0,003675 & -0,000748 \\
M & -0,001659 & 0,001348 & -0,000748 & +0,003589
\end{array}\right) \\
=\left(\begin{array}{ll}
\hat{d}_{c, 11} & \hat{d}_{(1)} \\
\hat{d}_{(1)}^{\prime} & \hat{D}_{22}
\end{array}\right)
\end{gathered}
$$

de onde se obtém:

$$
\begin{aligned}
& =1-\hat{R}_{1.234}^{2}=1-\frac{\hat{d}_{(1)} \hat{D}_{22}^{-1} \hat{d}_{(1)}^{\prime}}{\hat{d}_{c, 11}} \\
& =1-0,6398791=0,3601209
\end{aligned}
$$

2. Calcula-se a codependência múltipla da espécie 5 com as espécies $(2,3$, 4). Considera-se apenas a matriz de dependências e codependências entre as espécies $(5,2,3,4)$ particionada da seguinte maneira:

$$
\left(\begin{array}{rrrrr} 
& S & H & K & M \\
S & 0,002993 & 0,000385 & -0,001576 & -0,000949 \\
--- & --- & --- & --- & -- \\
H & 0,000385 & 0,005118 & -0,001111 & +0,001348 \\
K & -0,001576 & -0,001111 & +0,003675 & -0,000748 \\
M & -0,000949 & 0,001348 & -0,000748 & +0,003589
\end{array}\right)
$$




$$
=\left(\begin{array}{ll}
\hat{d}_{c, 55} & \hat{d}_{(5)} \\
\hat{d}_{(5)}^{\prime} & \hat{D}_{22}
\end{array}\right)
$$

de onde se obtém:

$$
\begin{aligned}
& 1-\hat{R}_{5.234}^{2}=1-\frac{\hat{d}_{(5)} \hat{D}_{22}^{-1} \hat{d}_{(5)}^{\prime}}{\hat{d}_{c, 55}} \\
& =1-0,3904588=0,6095412
\end{aligned}
$$

3. Calcula-se a co-dependência parcial das espécies ( 1 e 5), dadas as espécies $(2,3,4)$. Ela é calculada a partir da matriz de co-dependências condicionais de (1 e 5) dadas as restantes, segundo a seguinte partição:

$$
\begin{aligned}
& \left(\begin{array}{cccccc} 
& A=1 & S=5 & H & K & M \\
A & 0,003902 & -0,000348 & -0,002909 & -0,000662 & -0,001659 \\
S & -0,000348 & 0,002993 & 0,000385 & -0,001576 & -0,000949 \\
-- & -- & -- & -- & -- & \\
H & -0,002909 & 0,000385 & 0,005118 & -0,001111 & 0,001348 \\
K & -0,000662 & -0,001576 & -0,001111 & 0,003675 & -0,000748 \\
M & -0,001659 & -0,000949 & 0,001348 & -0,000748 & 0,003589
\end{array}\right) \\
& =\left(\begin{array}{cc}
\hat{D}_{11} & \hat{D}_{12} \\
& \\
\hat{D}_{21} & \hat{D}_{22}
\end{array}\right)
\end{aligned}
$$

de onde obtém-se

$$
\begin{aligned}
& \hat{D}_{11.2}=\hat{D}_{11}-\hat{D}_{12} \hat{D}_{22}^{-1} \hat{D}_{21} \\
& =\left(\begin{array}{cc}
0,001405 & -0,001145 \\
-0,001145 & 0,001824
\end{array}\right) \\
& =\left(\begin{array}{cc}
\hat{d}_{c ; 11.234} & \hat{d}_{c ; 15.234} \\
\hat{d}_{c ; 51.234} & \hat{d}_{c ; 55.234}
\end{array}\right) \\
& \hat{\varrho}_{15.234}=\frac{-0,001145}{\sqrt{0,001405 \overline{3} 0,0018241}}=-0,715232
\end{aligned}
$$


4. Calcula-se o coeficiente de trajetória desde a espécie A (1) até a espécie S (5)

$$
\begin{aligned}
& \hat{\wp}_{51}=\hat{\varrho}_{51.234} \sqrt{\frac{1-\hat{R}_{5.234}^{2}}{1-\hat{R}_{1.234}^{2}}} \\
& =-0,715232 \sqrt{\frac{0,6095412}{0,3601209}}=-0,930517
\end{aligned}
$$

assim,

$$
\hat{\wp}_{51}=-0,930517
$$

ii) Obtenção do caminho a partir da espécie $H$ até a espécie $S$ (de 2 até 5)

1. Calcula-se a codependência múltipla da espécie 2 com as espécies $(1,3$, 4). Considera-se somente a matriz de dependências e codependências entre as espécies $(2,1,3,4)$ subdividida da seguinte maneira:

$$
\begin{aligned}
& \left(\begin{array}{ccccc} 
& 2 & 1 & 3 & 4 \\
2 & 0,005118 & -0,002909 & -0,00111 & 0,001348 \\
-- & -- & -- & -- & \\
1 & -0,002909 & 0,003902 & -0,000662 & -0,001659 \\
3 & -0,00111 & -0,000662 & +0,003675 & -0,000748 \\
4 & -0,001348 & -0,001659 & -0,000748 & +0,003589
\end{array}\right) \\
& =\left(\begin{array}{ll}
\hat{d}_{c, 11} & \hat{d}_{(1)} \\
\hat{d}_{(1)}^{\prime} & \hat{D}_{22}
\end{array}\right)
\end{aligned}
$$

de onde se obtém

$$
\begin{aligned}
& =1-\hat{R}_{2.134}^{2}=1-\frac{\hat{d}_{(1)} \hat{D}_{22}^{-1} \hat{d}_{(1)}^{\prime}}{\hat{d}_{c, 11}} \\
& =1-0,574112=0,425888
\end{aligned}
$$


2. Calcula-se a codependência múltipla da espécie 5 com as espécies $(1,3$, 4). Considera-se somente a matriz de dependências e codependências entre as espécies $(5,1,3,4)$ subdividida da seguinte maneira:

$$
\begin{gathered}
\left(\begin{array}{ccccc} 
& S & A & K & M \\
S & 0,002993 & -0,000348 & -0,001576 & -0,000949 \\
-- & -- & -- & -- & - \\
A & -0,000348 & 0,003902 & -0,000662 & -0,001659 \\
K & -0,001576 & -0,000662 & +0,003675 & -0,000748 \\
M & -0,000949 & -0,001659 & -0,000748 & +0,003589
\end{array}\right) \\
=\left(\begin{array}{cc}
\hat{d}_{c, 55} & \hat{d}_{(5)} \\
\hat{d}_{(5)}^{\prime} & \hat{D}_{22}
\end{array}\right)
\end{gathered}
$$

de onde se obtém

$$
\begin{aligned}
& 1-\hat{R}_{5.134}^{2}=1-\frac{\hat{d}_{(5)} \hat{D}_{22}^{-1} \hat{d}_{(5)}^{\prime}}{\hat{d}_{c, 55}} \\
& =1-0,5902176=0,4097824
\end{aligned}
$$

3. Calcula-se a codependência parcial das espécies (2 e 5) dadas as espécies $(1,3,4)$. Ela é calculada a partir da matriz de codependências condicionais de (2 e 5) dadas as restantes, segundo a seguinte partição:

$$
\begin{aligned}
&\left(\begin{array}{cccccc} 
& H=2 & S=5 & A & K & M \\
H & 0,005118 & 0,000385 & -0,002909 & -0,00111 & 0,001348 \\
S & 0,000385 & 0,002993 & -0,000348 & -0,001576 & -0,000949 \\
- & - & -- & -- & -- & -- \\
A & -0,002909 & -0,000384 & 0,003902 & -0,000662 & +0,001659 \\
K & -0,00111 & -0,001576 & -0,000662 & +0,003675 & -0,000748 \\
M & 0,001348 & -0,000949 & -0,001659 & -0,000748 & +0,003589
\end{array}\right) \\
&=\left(\begin{array}{cc}
\hat{D}_{11} & \hat{D}_{12} \\
\hat{D}_{21} & \hat{D}_{22}
\end{array}\right) \\
&
\end{aligned}
$$


de onde se obtém

$$
\begin{aligned}
& \hat{D}_{11.2}=\hat{D}_{11}-\hat{D}_{12} \hat{D}_{22}^{-1} \hat{D}_{21} \\
& =\left(\begin{array}{cc}
+0,0021796 & -0,000855 \\
--- & --- \\
-0,000855 & 0,0012263
\end{array}\right) \\
& =\left(\begin{array}{cc}
\hat{d}_{c ; 22.134} & \hat{d}_{c ; 25.134} \\
\hat{d}_{c ; 52.134} & \hat{d}_{c ; 55.134}
\end{array}\right) \\
& \hat{\varrho}_{25.134}=\frac{-0,000855}{\sqrt{0,00217960,0012263}}=-0,522927
\end{aligned}
$$

4. Calcula-se o coeficiente de trajetória desde a espécie $H(2)$ até a espécie S (5)

$$
\begin{aligned}
& \hat{\wp}_{52}=\hat{\varrho}_{52.134} \sqrt{\frac{1-\hat{R}_{5.134}^{2}}{1-\hat{R}_{2.2134}^{2}}} \\
& =-0,522927 \sqrt{\frac{0,4097824}{0,425888}}=-0,512944
\end{aligned}
$$

assim,

$$
\hat{\wp}_{52}=-0,5129441
$$

iii) Da mesma maneira calculam-se, o coeficiente de trajetória desde a espécie $\mathrm{K}$ (3) até a espécie $S(5)$

$$
\begin{aligned}
& \hat{\wp}_{53}=\hat{\varrho}_{53.124} \sqrt{\frac{1-\hat{R}_{5.124}^{2}}{1-\hat{R}_{3.124}^{2}}} \\
& =-0,803926 \sqrt{\frac{0,8417428}{0,6436002}}=-0,919386
\end{aligned}
$$

assim,

$$
\hat{\wp}_{53}=-0,919386
$$


iv) O coeficiente de trajetória desde a espécie $M(4)$ até a espécie $S$ (5)

$$
\begin{aligned}
& \hat{\wp}_{54}=\hat{\varrho}_{54.123} \sqrt{\frac{1-\hat{R}_{5.123}^{2}}{1-\hat{R}_{4123}^{2}}} \\
& =-0,746953 \sqrt{\frac{0,6734959}{0,7053009}}=-0,729917 \\
& \hat{\wp}_{54}=-0,729917
\end{aligned}
$$

a) Resumindo, tem-se a estimativa do vetor de coeficientes de trajetórias desde as outras espécies até a espécie Simulans (S ou 5):

$$
\left(\begin{array}{l}
\hat{\wp}_{51} \\
\hat{\wp}_{52} \\
\hat{\wp}_{53} \\
\hat{\wp}_{54}
\end{array}\right)=\left(\begin{array}{l}
\hat{\wp}_{S A} \\
\hat{\wp}_{S H} \\
\hat{\wp}_{S K} \\
\hat{\wp}_{S M}
\end{array}\right)=\left(\begin{array}{c}
-0,930517 \\
-0,512944 \\
-0,919386 \\
-0,729917
\end{array}\right)
$$

Seguindo a mesma metodologia e fazendo as partições adequadas da matriz de coeficientes de codependência, são calculados os outros coeficientes de trajeórias. Têm-se, portanto,

b) os coeficientes de trajetórias das outras espécies à espécie Melanogaster $(M=4)$ :

$$
\left(\begin{array}{l}
\hat{\wp}_{41} \\
\hat{\wp}_{42} \\
\hat{\wp}_{43} \\
\hat{\wp}_{45}
\end{array}\right)=\left(\begin{array}{l}
\hat{\wp}_{M A} \\
\hat{\wp}_{M H} \\
\hat{\wp}_{M K} \\
\hat{\wp}_{M S}
\end{array}\right)=\left(\begin{array}{c}
-0,98956 \\
-0,475378 \\
-0,863752 \\
-0,764388
\end{array}\right)
$$

c) os coeficientes de trajetórias das outras espécies à espécie Kikkawai $(K=3)$ :

$$
\left(\begin{array}{l}
\hat{\wp}_{31} \\
\hat{\wp}_{32} \\
\hat{\wp}_{34} \\
\hat{\wp}_{35}
\end{array}\right)=\left(\begin{array}{l}
\hat{\wp}_{K A} \\
\hat{\wp}_{K H} \\
\hat{\wp}_{K M} \\
\hat{\wp}_{K S}
\end{array}\right)=\left(\begin{array}{c}
-0,912335 \\
-0,582207 \\
-0,630646 \\
-0,702967
\end{array}\right)
$$


d) os coeficientes de trajetórias das outras espécies à espécie Kikkawai $(\mathrm{H}=2)$ :

$$
\left(\begin{array}{l}
\hat{\wp}_{21} \\
\hat{\wp}_{23} \\
\hat{\wp}_{24} \\
\hat{\wp}_{25}
\end{array}\right)=\left(\begin{array}{l}
\hat{\wp}_{H A} \\
\hat{\wp}_{H K} \\
\hat{\wp}_{H M} \\
\hat{\wp}_{H S}
\end{array}\right)=\left(\begin{array}{c}
-1,058343 \\
-0,791378 \\
-0,471783 \\
-0,533105
\end{array}\right)
$$

e) os coeficientes de trajetórias das outras espécies à espécie Ananassae $(A=1)$ :

$$
\left(\begin{array}{l}
\hat{\wp}_{12} \\
\hat{\wp}_{13} \\
\hat{\wp}_{14} \\
\hat{\wp}_{15}
\end{array}\right)=\left(\begin{array}{l}
\hat{\wp}_{A H} \\
\hat{\wp}_{A K} \\
\hat{\wp}_{A M} \\
\hat{\wp}_{A S}
\end{array}\right)=\left(\begin{array}{l}
-0,601629 \\
-0,704957 \\
-0,558275 \\
-0,549755
\end{array}\right)
$$

\subsection{Decomposição dos coeficientes de co-dependência}

Segundo o teorema fundamental da análise de trajetória, pode-se decompor os coeficientes de co-dependência entre dois níveis da espécie Drosophyla em seus componentes:

1. os efeitos diretos $\mathrm{e}$

2. os outros efeitos.

O primeiro é medido pelas estimativas dos coeficientes de trajetórias correspondentes e os outros efeitos são obtidos por diferença. Assim, segundo o Teorema Fundamental proposto no item (3.3.2) têm-se:

$$
\begin{aligned}
& \hat{\delta}_{12}=\sum_{i=1}^{12} Z_{1 i} Z_{2 i}=\sum_{i=1}^{12}\left(\hat{\wp}_{12} Z_{2 i}+\hat{\wp}_{13} Z_{3 i}+\hat{\wp}_{14} Z_{4 i}+\hat{\wp}_{15} Z_{5}\right) Z_{2 i} \\
& =\hat{\wp}_{12}+\hat{\wp}_{13} \hat{\delta}_{32}+\hat{\wp}_{14} \hat{\delta}_{42}+\hat{\wp}_{15} \hat{\delta}_{52}=====>\hat{\delta}_{12}=-0,650986 \\
& \hat{\delta}_{13}=\sum_{i=1}^{12} Z_{1 i} Z_{3 i}=\sum_{i=1}^{12}\left(\hat{\wp}_{12} Z_{2 i}+\hat{\wp}_{13} Z_{3 i}+\hat{\wp}_{14} Z_{4 i}+\hat{\wp}_{15} Z_{5}\right) Z_{3 i} \\
& =\hat{\wp}_{13}+\hat{\wp}_{12} \hat{\delta}_{23}+\hat{\wp}_{14} \hat{\delta}_{43}+\hat{\wp}_{15} \hat{\delta}_{53}=====>\hat{\delta}_{13}=-0,174852 \\
& \hat{\delta}_{14}=\sum_{i=1}^{12} Z_{1 i} Z_{4 i}=\sum_{i=1}^{12}\left(\hat{\wp}_{12} Z_{2 i}+\hat{\wp}_{13} Z_{3 i}+\hat{\wp}_{14} Z_{4 i}+\hat{\wp}_{15} Z_{5}\right) Z_{4 i}
\end{aligned}
$$


$=\hat{\wp}_{14}+\hat{\wp}_{12} \hat{\delta}_{24}+\hat{\wp}_{13} \hat{\delta}_{34}+\hat{\wp}_{15} \hat{\delta}_{54}=====>\hat{\delta}_{14}=-0,443187$ $\hat{\delta}_{15}=\sum_{i=1}^{12} Z_{1 i} Z_{5 i}=\sum_{i=1}^{12}\left(\hat{\wp}_{12} Z_{2 i}+\hat{\wp}_{13} Z_{3 i}+\hat{\wp}_{14} Z_{4 i}+\hat{\wp}_{15} Z_{5}\right) Z_{5 i}$ $=\hat{\wp}_{15}+\hat{\wp}_{12} \hat{\delta}_{25}+\hat{\wp}_{13} \hat{\delta}_{35}+\hat{\wp}_{14} \hat{\delta}_{45}====>\hat{\delta}_{15}=-0,112351$ $\hat{\delta}_{21}=\sum_{i=1}^{12} Z_{2 i} Z_{1 i}=\sum_{i=1}^{12}\left(\hat{\wp}_{21} Z_{1 i}+\hat{\wp}_{23} Z_{3 i}+\hat{\wp}_{24} Z_{4 i}+\hat{\wp}_{25} Z_{5}\right) Z_{1 i}$ $=\hat{\wp}_{21}+\hat{\wp}_{23} \hat{\delta}_{31}+\hat{\wp}_{24} \hat{\delta}_{41}+\hat{\wp}_{25} \hat{\delta}_{51}=====>\hat{\delta}_{21}=-0,650986$ $\hat{\delta}_{23}=\sum_{i=1}^{12} Z_{2 i} Z_{3 i}=\sum_{i=1}^{12}\left(\hat{\wp}_{21} Z_{1 i}+\hat{\wp}_{23} Z_{3 i}+\hat{\wp}_{24} Z_{4 i}+\hat{\wp}_{25} Z_{5}\right) Z_{3 i}$ $=\hat{\wp}_{23}+\hat{\wp}_{21} \hat{\delta}_{13}+\hat{\wp}_{24} \hat{\delta}_{43}+\hat{\wp}_{25} \hat{\delta}_{53}====>\hat{\delta}_{23}=-0,255897$ $\hat{\delta}_{24}=\sum_{i=1}^{12} Z_{2 i} Z_{4 i}=\sum_{i=1}^{12}\left(\hat{\wp}_{21} Z_{1 i}+\hat{\wp}_{23} Z_{3 i}+\hat{\wp}_{24} Z_{4 i}+\hat{\wp}_{25} Z_{5}\right) Z_{4 i}$ $=\hat{\wp}_{24}+\hat{\wp}_{21} \hat{\delta}_{14}+\hat{\wp}_{23} \hat{\delta}_{34}+\hat{\wp}_{25} \hat{\delta}_{54}=====>\hat{\delta}_{24}=0,314579$ $\hat{\delta}_{25}=\sum_{i=1}^{12} Z_{2 i} Z_{5 i}=\sum_{i=1}^{12}\left(\hat{\wp}_{21} Z_{1 i}+\hat{\wp}_{23} Z_{3 i}+\hat{\wp}_{24} Z_{4 i}+\hat{\wp}_{25} Z_{5}\right) Z_{5 i}$ $=\hat{\wp}_{25}+\hat{\wp}_{21} \hat{\delta}_{15}+\hat{\wp}_{23} \hat{\delta}_{35}+\hat{\wp}_{24} \hat{\delta}_{45}=====>\hat{\delta}_{25}=0,098453$ $\hat{\delta}_{31}=\sum_{i=1}^{12} Z_{3 i} Z_{1 i}=\sum_{i=1}^{12}\left(\hat{\wp}_{31} Z_{1 i}+\hat{\wp}_{32} Z_{2 i}+\hat{\wp}_{34} Z_{4 i}+\hat{\wp}_{35} Z_{5}\right) Z_{1 i}$ $=\hat{\wp}_{31}+\hat{\wp}_{32} \hat{\delta}_{21}+\hat{\wp}_{34} \hat{\delta}_{41}+\hat{\wp}_{35} \hat{\delta}_{51}====>\hat{\delta}_{31}=-0,174852$ $\hat{\delta}_{32}=\sum_{i=1}^{12} Z_{3 i} Z_{2 i}=\sum_{i=1}^{12}\left(\hat{\wp}_{31} Z_{1 i}+\hat{\wp}_{32} Z_{2 i}+\hat{\wp}_{34} Z_{4 i}+\hat{\wp}_{35} Z_{5}\right) Z_{2 i}$ $=\hat{\wp}_{32}+\hat{\wp}_{31} \hat{\delta}_{12}+\hat{\wp}_{34} \hat{\delta}_{42}+\hat{\wp}_{35} \hat{\delta}_{52}====>\hat{\delta}_{32}=-0,255887$ $\hat{\delta}_{34}=\sum_{i=1}^{12} Z_{3 i} Z_{4 i}=\sum_{i=1}^{12}\left(\hat{\wp}_{31} Z_{1 i}+\hat{\wp}_{32} Z_{2 i}+\hat{\wp}_{34} Z_{4 i}+\hat{\wp}_{35} Z_{5}\right) Z_{4 i}$ $=\hat{\wp}_{34}+\hat{\wp}_{31} \hat{\delta}_{14}+\hat{\wp}_{32} \hat{\delta}_{24}+\hat{\wp}_{35} \hat{\delta}_{54}====>\hat{\delta}_{34}=-0,205883$ $\hat{\delta}_{35}=\sum_{i=1}^{12} Z_{3 i} Z_{5 i}=\sum_{i=1}^{12}\left(\hat{\wp}_{31} Z_{1 i}+\hat{\wp}_{32} Z_{2 i}+\hat{\wp}_{34} Z_{4 i}+\hat{\wp}_{35} Z_{5}\right) Z_{5 i}$ $=\hat{\wp}_{35}+\hat{\wp}_{31} \hat{\delta}_{15}+\hat{\wp}_{32} \hat{\delta}_{25}+\hat{\wp}_{34} \hat{\delta}_{45}=====>\hat{\delta}_{35}=-0,475150$ $\hat{\delta}_{41}=\sum_{i=1}^{12} Z_{4 i} Z_{1 i}=\sum_{i=1}^{12}\left(\hat{\wp}_{41} Z_{1 i}+\hat{\wp}_{42} Z_{2 i}+\hat{\wp}_{43} Z_{3 i}+\hat{\wp}_{45} Z_{5}\right) Z_{1 i}$ $=\hat{\wp}_{41}+\hat{\wp}_{42} \hat{\delta}_{21}+\hat{\wp}_{43} \hat{\delta}_{31}+\hat{\wp}_{45} \hat{\delta}_{51}====>\hat{\delta}_{41}=-0,443187$ $\hat{\delta}_{42}=\sum_{i=1}^{12} Z_{4 i} Z_{2 i}=\sum_{i=1}^{12}\left(\hat{\wp}_{41} Z_{1 i}+\hat{\wp}_{42} Z_{2 i}+\hat{\wp}_{43} Z_{3 i}+\hat{\wp}_{45} Z_{5}\right) Z_{2 i}$ $=\hat{\wp}_{42}+\hat{\wp}_{41} \hat{\delta}_{12}+\hat{\wp}_{43} \hat{\delta}_{32}+\hat{\wp}_{45} \hat{\delta}_{52}====>\hat{\delta}_{42}=0,314579$ $\hat{\delta}_{43}=\sum_{i=1}^{12} Z_{4 i} Z_{3 i}=\sum_{i=1}^{12}\left(\hat{\wp}_{41} Z_{1 i}+\hat{\wp}_{42} Z_{2 i}+\hat{\wp}_{43} Z_{3 i}+\hat{\wp}_{45} Z_{5}\right) Z_{3 i}$ $=\hat{\wp}_{43}+\hat{\wp}_{41} \hat{\delta}_{13}+\hat{\wp}_{42} \hat{\delta}_{23}+\hat{\wp}_{45} \hat{\delta}_{53}====>\hat{\delta}_{43}=0,205883$ $\hat{\delta}_{45}=\sum_{i=1}^{12} Z_{4 i} Z_{5 i}=\sum_{i=1}^{12}\left(\hat{\wp}_{41} Z_{1 i}+\hat{\wp}_{42} Z_{2 i}+\hat{\wp}_{43} Z_{3 i}+\hat{\wp}_{45} Z_{5}\right) Z_{5 i}$ 
$=\hat{\wp}_{45}+\hat{\wp}_{41} \hat{\delta}_{15}+\hat{\wp}_{42} \hat{\delta}_{25}+\hat{\wp}_{43} \hat{\delta}_{35}=====>\hat{\delta}_{45}=-0,289599$ $\hat{\delta}_{51}=\sum_{i=1}^{12} Z_{5 i} Z_{1 i}=\sum_{i=1}^{12}\left(\hat{\wp}_{51} Z_{1 i}+\hat{\wp}_{52} Z_{2 i}+\hat{\wp}_{53} Z_{3 i}+\hat{\wp}_{54} Z_{4}\right) Z_{1 i}$

$=\hat{\wp}_{51}+\hat{\wp}_{52} \hat{\delta}_{21}+\hat{\wp}_{53} \hat{\delta}_{31}+\hat{\wp}_{54} \hat{\delta}_{41}====>\hat{\delta}_{51}=-0,112351$ $\hat{\delta}_{52}=\sum_{i=1}^{12} Z_{5 i} Z_{2 i}=\sum_{i=1}^{12}\left(\hat{\wp}_{51} Z_{1 i}+\hat{\wp}_{52} Z_{2 i}+\hat{\wp}_{53} Z_{3 i}+\hat{\wp}_{54} Z_{4}\right) Z_{2 i}$

$=\hat{\wp}_{52}+\hat{\wp}_{51} \hat{\delta}_{12}+\hat{\wp}_{53} \hat{\delta}_{32}+\hat{\wp}_{54} \hat{\delta}_{42}====>\hat{\delta}_{52}=0,098453$ $\hat{\delta}_{53}=\sum_{i=1}^{12} Z_{5 i} Z_{3 i}=\sum_{i=1}^{12}\left(\hat{\wp}_{51} Z_{1 i}+\hat{\wp}_{52} Z_{2 i}+\hat{\wp}_{53} Z_{3 i}+\hat{\wp}_{54} Z_{4}\right) Z_{3 i}$

$=\hat{\wp}_{53}+\hat{\wp}_{51} \hat{\delta}_{13}+\hat{\wp}_{52} \hat{\delta}_{23}+\hat{\wp}_{54} \hat{\delta}_{43}====>\hat{\delta}_{53}=-0,47515$ $\hat{\delta}_{54}=\sum_{i=1}^{12} Z_{5 i} Z_{4 i}=\sum_{i=1}^{12}\left(\hat{\wp}_{51} Z_{1 i}+\hat{\wp}_{52} Z_{2 i}+\hat{\wp}_{53} Z_{3 i}+\hat{\wp}_{54} Z_{4}\right) Z_{4 i}$ $=\hat{\wp}_{54}+\hat{\wp}_{51} \hat{\delta}_{14}+\hat{\wp}_{52} \hat{\delta}_{24}+\hat{\wp}_{53} \hat{\delta}_{34}====>\hat{\delta}_{54}=-0,289599$

Assim, por exemplo, a associação direta das espécies Hydei e Ananassae é dado por $\hat{\wp}_{12}=-0,6016$. Os outros efeitos são determinados por diferença: $\hat{\delta}_{12}-\hat{\wp}_{12}, \operatorname{com} \hat{\delta}_{12}$ determinado pelo teorema fundamental.

Além disso têm-se as associações que se denominou por efeitos residuais, estimados segundo (79). Assim:

$\hat{\wp}_{1 a}^{2}=1-\sum_{j=2,5} \hat{\wp}_{1 j} \hat{\delta}_{1 j}$

$=1-\hat{R}_{1.2345}^{2}=0,1759=====>\hat{\wp} \hat{\wp}_{1 a}=0,4194$

$\hat{\wp}_{2 b}^{2}=1-\sum_{j=1,5} \hat{\wp}_{2 j} \hat{\delta}_{2 j}$

$=1-\hat{R}_{2.1345}^{2}=0,3094====>\hat{\wp}_{2 b}=0,5563$

$\hat{\wp}_{3 c}^{2}=1-\sum_{j=1,5} \hat{\wp}_{3 j} \hat{\delta}_{3 j}$

$=1-\hat{R}_{3.1245}^{2}=0,2276====>\hat{\hat{\wp}_{3 c}=0,4771}$

$\hat{\wp}_{4 d}^{2}=1-\sum_{j=1,5} \hat{\wp}_{4 j} \hat{\delta}_{4 j}$

$=1-\hat{R}_{4.1235}^{2}=0,3118=====>\hat{\wp}_{4 d}=0,55838$

$\hat{\wp}_{5 e}^{2}=1-\sum_{j=1,4} \hat{\wp}_{5 j} \hat{\delta}_{5 j}$

$=1-\hat{R}_{5.1234}^{2}=0,2977====>\hat{\wp}_{5 e}=0,54564$

Os coeficientes de trajetória podem ser, portanto, usados para reproduzir os coeficientes de co-dependência observados entre os diferentes níveis da espécie Drosophyla, obtidos também através de ANADEP.

A associação direta entre as espécies: 
Hydei e Ananassae é dada por $\hat{\wp}_{12}=-0,6016$;

Kikkawai e Ananassae é dada por $\hat{\wp}_{13}=-0,7049$;

Melanogaster e Ananassae é dada por $\hat{\wp}_{14}=-0,5583$;

Sinulans e Ananassae é dada por $\hat{\wp}_{15}=-0,5498$.

Os outros coeficientes de trajetória são interpretados da mesma maneira.

O diagrama de trajetórias associado, já com os coeficientes estimados é o da Figura 7 e o resumo da decomposição dos coeficientes de co-dependência é apresentado na Tabela 10.

\subsection{Interterpretações dos coeficientes de trajetórias}

Os resultados obtidos nas seções (4.3) e (4.4), sugerem que é razoável admitir as seguintes interpretaçōes:

Todas as outras espécies de moscas têm repulsa pela espécie Ananassae (1), pois os ambientes ou leveduras que esta mais prefere não são escolhidos pelas outras espécies, mas, ela poderia estar nos ambientes que são preferidos pelas outras espécies. Observa-se que os coeficientes de trajetória desde as outras espécies à espécie Ananassae(1), $\hat{\wp}_{1 j}, j=2,3,4,5$, são bastante menores quando comparados com os correspondentes coeficientes de trajetória $\hat{\wp}_{i 1}, i=2,3,4,5$.

As espécies Simulans (5) e Hydei (2) tendem a preferir a mesma levedura como primeira opção. Assim os coeficientes $\hat{\wp}_{52}=-0,5130$ e $\hat{\wp}_{25}=-0,5331$ são bastante parecidos.

As espécies Melanogaster (4) e Simulans(5) tembém tendem a preferir as mesmas leveduras, assim têm-se que os correspondentes coeficientes $\hat{\wp}_{45}=-0,7644$ e $\hat{\wp}_{54}=-0,7299$ são similares.

As espécies Hydei (2) e Kikkawai (3) poderiam ter preferências por ambientes diferentes, ou a escolha dos ambientes poderia ser feita não necessariamente na mesma ordem. Assim, se Hydei preferir as leveduras L6-L11, a espécie Kikkawai escolheria essa levedura em terceira ou quarta opção pois os coeficientes $\hat{\wp}_{23}=-0,7914$ e $\hat{\wp}_{32}=-0,5822$ são diferentes. 
FIGURA 7: Gráfico de Trajetória para as espécies Drosophyla

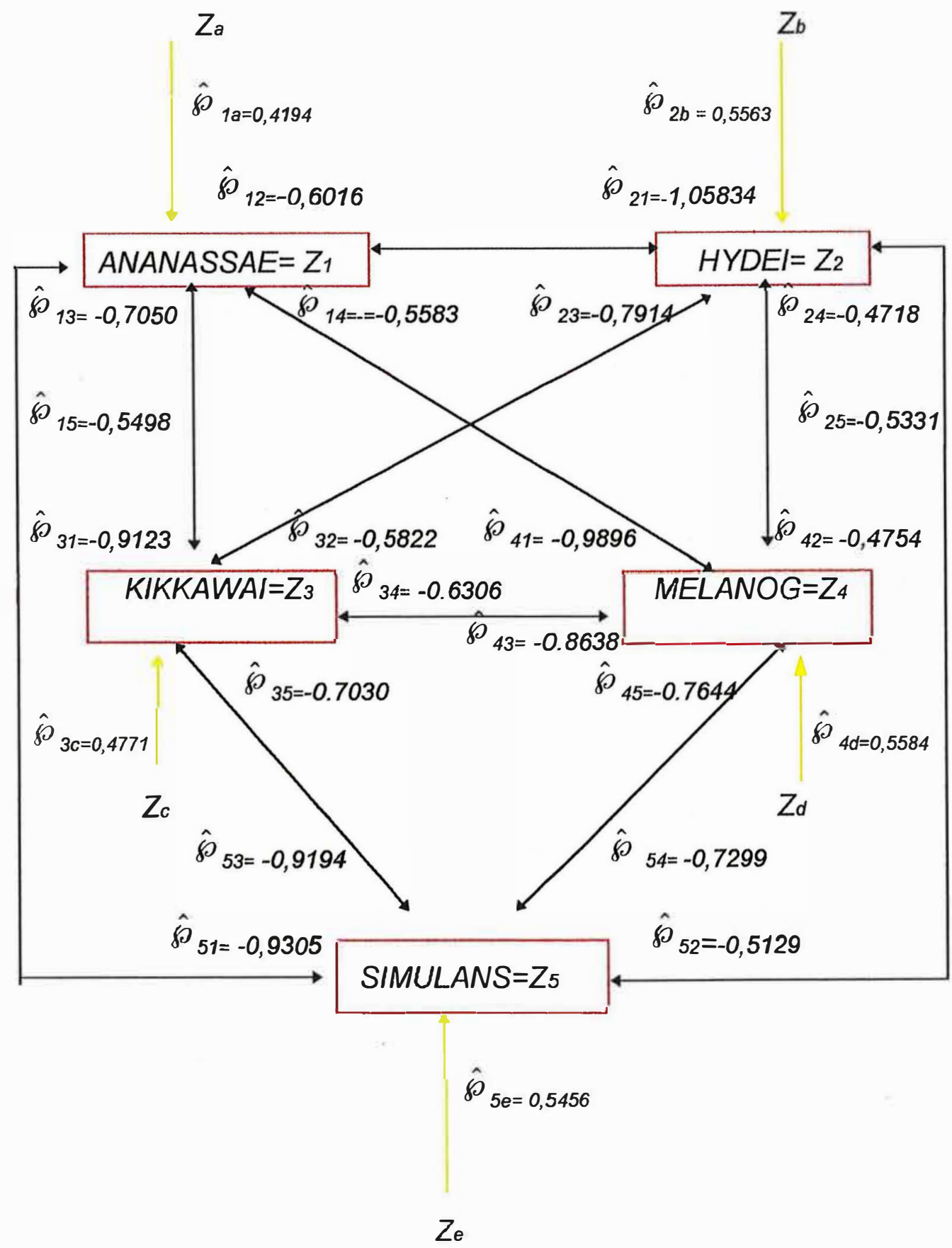


TABELA 10: Decomposição dos Coeficientes de Co-dependência

\begin{tabular}{ccc}
\hline Co-dependências & \multicolumn{2}{c}{ Coeficientes de Associação } \\
& Diretos & Outros \\
\hline$\hat{\delta}_{12}=-0,6510$ & $\hat{\wp}_{12}=-0,6016$ & $-0,0494$ \\
$\hat{\delta}_{13}=-0,1748$ & $\hat{\wp}_{13}=-0,7049$ & 0,5301 \\
$\hat{\delta}_{14}=-0,4432$ & $\hat{\wp}_{14}=-0,5583$ & 0,1151 \\
$\hat{\delta}_{15}=-0,1124$ & $\hat{\wp}_{15}=-0,5498$ & 0,4374 \\
$\hat{\delta}_{21}=-0,6510$ & $\hat{\wp}_{21}=-1,0583$ & 0,4070 \\
$\hat{\delta}_{23}=-0,2559$ & $\hat{\wp}_{23}=-0,7914$ & 0,5355 \\
$\hat{\delta}_{24}=0,3146$ & $\hat{\wp}_{24}=-0,4718$ & 0,7864 \\
$\hat{\delta}_{25}=0,0985$ & $\hat{\wp}_{25}=-0,5331$ & 0,6316 \\
$\hat{\delta}_{31}=-0,1749$ & $\hat{\wp}_{31}=-0,9123$ & 0,7374 \\
$\hat{\delta}_{32}=-0,2559$ & $\hat{\wp}_{32}=-0,5822$ & 0,3263 \\
$\hat{\delta}_{34}=-0,2059$ & $\hat{\wp}_{34}=-0,6306$ & 0,4247 \\
$\hat{\delta}_{35}=-0,4752$ & $\hat{\wp}_{35}=-0,7030$ & 0,2278 \\
$\hat{\delta}_{41}=-0,4432$ & $\hat{\wp}_{41}=-0,9896$ & 0,5464 \\
$\hat{\delta}_{42}=0,3146$ & $\hat{\wp}_{42}=-0,4754$ & 0,7900 \\
$\hat{\delta}_{43}=-0,2059$ & $\hat{\wp}_{43}=-0,8638$ & 0,6579 \\
$\hat{\delta}_{45}=-0,2896$ & $\hat{\wp}_{45}=-0,7644$ & 0,4748 \\
$\hat{\delta}_{51}=-0,1124$ & $\hat{\wp}_{51}=-0,9305$ & 0,8181 \\
$\hat{\delta}_{52}=0,0985$ & $\hat{\wp}_{52}=-0,5129$ & 0,6114 \\
$\hat{\delta}_{53}=-0,4752$ & $\hat{\wp}_{53}=-0,9194$ & 0,4442 \\
$\hat{\delta}_{54}=-0,2896$ & $\hat{\wp}_{54}=-0,7299$ & 0,4403 \\
\hline & &
\end{tabular}


A proximidade entre os coeficientes $\hat{\wp}_{24}=-0,471783$ e $\hat{\wp}_{42}=$ $-0,475378$ poderia ser explicado pelo fato que as espécies Hydei (2) e Melanogaster (4) foram as únicas espécies que escolheram as leveduras L12-L14, mesmo que seja em ordens de preferência diferentes, pois isso foi observado ao comparar os perfis correspondentes.

As espécies Kikkawai (3) e Melanogaster (4), apesar de terem as mesmas preferências iniciais elas escolhem leveduras diferentes, isso poderia explicar o fato de os coeficientes de trajetória $\hat{\wp}_{34}=-0,630646$ e $\hat{\wp}_{43}=-0,863752$ serem diferentes.

Em geral, pode-se dizer que coeficientes parecidos estariam indicando preferência das espécies de moscas pelos mesmos ambientes ou leveduras, entretanto, coeficientes diferentes poderiam indicar escolha de ambientes também diferentes.

Cabe sinalar que as interpretações ainda precisam ser mais exploradas, mas, as consideradas aqui são compatíveis com os resultados descritivos, perfis probabilísticos e gráficos dos fatores (, Cordeiro, 1990).

\subsection{Testes de hipóteses}

A partir dos resultados anteriores, pode ser de interesse testar algumas hipóteses a respeito dos coeficientes de trajetória.

Assim, para a função de regressão

$$
\begin{aligned}
& Z_{1 i}=0,6016 Z_{2 i}-0,7049 Z_{3 i}-0,5583 Z_{4 i}-0,5497 Z_{5 i} \\
& i=1,2, \ldots, 12
\end{aligned}
$$

pode ser de interesse testar a hipótese

$$
H_{0}: \wp_{14}=0 .
$$

Segundo a proposta dada por (3.4), obtêm-se os seguintes resultados: 
- $\hat{R}_{m}^{2}=1-\left(1-\hat{R}_{1.2345}^{2}\right)=0,824102$

- $M=1-\left(1-\hat{R}_{1.235}^{2}\right)=0,6069775$

- $W=-11 \operatorname{Ln}\left(\frac{1-\hat{R}_{m}^{2}}{1-M}\right)=-11 \operatorname{Ln}(0.1759 / 0.3930)=8,8435$

- $X_{(5 \%, 1)}=3,8435$, com $s=1$ ( número de restrições)

- Logo, a hipótese $H_{0}$ é rejeitada.

Seguindo-se a mesma metodologia têm-se os seguintes resultados, para cada uma das possíveis hipóteses testadas, mostrados nas tabelas 11, 12, 13, 14 e 15 .

Se os resultados fornecidos pelos testes anteriores fossem válidos, ter-se-ia a seguinte situação:

$$
\begin{array}{llll}
Z_{1}=f\left(Z_{2}, Z_{3}, Z_{4}, Z_{5}\right) & \text { com } & \hat{R}_{1.2345}^{2}=0,8241 \\
Z_{2}=f\left(Z_{1}\right) & \text { com } & \hat{R}_{2.1}^{2}=0,4238 \\
Z_{3}=f\left(Z_{1}, Z_{2}, Z_{4}, Z_{5}\right) & \text { com } & \hat{R}_{3.1245}^{2}=0,7724 \\
Z_{4}=f\left(Z_{1}, Z_{3}, Z_{5}\right) & \text { com } & \hat{R}_{4.135}^{2}=0,5981 \\
Z_{5}=f\left(Z_{1}, Z_{3}, Z_{4}\right) & \text { com } & \hat{R}_{5.134}^{2}=0,5902
\end{array}
$$

Assim,

$$
\begin{aligned}
& Z_{1 i}=-0,016 Z_{2 i}-0,7050 Z_{3 i}-0,5583 Z_{4 i}-0,5498 Z_{5 i} \\
& Z_{2 i}=-0,6510 Z_{1 i}
\end{aligned}
$$

com efeito residual $\hat{\wp}_{2 b}=\left(1-\hat{R}_{2.1}^{2}\right)^{1 / 2}=0,7591$;

$$
\begin{aligned}
& Z_{3 i}=-0,9123 Z_{1 i}-0,5822 Z_{2 i}-0,6306 Z_{4 i}-0,7030 Z_{5 i} \\
& Z_{4 i}=-0,6271 Z_{1 i}-0,6285 Z_{3 i}-0,6587 Z_{5 i}
\end{aligned}
$$

com efeito residual $\hat{\wp}_{4 d}=\left(1-\hat{R}_{4.135}^{2}\right)^{1 / 2}=0,6340$;

$$
Z_{5 i}=-0,5335 Z_{1 i}-0,7067 Z_{3 i}-0,6716 Z_{4 i}
$$


TABELA 11: Resultados dos Testes de Hipóteses para a equação ( 81)

\begin{tabular}{rlrrl}
\hline Hipótese & $\hat{R}_{1 . l}^{2}$ & $I-s$ & $\begin{array}{r}W=(I-s) * \\
L n\left(\frac{1-\hat{R}_{1.245}^{2}}{1-\hat{R}_{1 .}^{2}}\right)\end{array}$ & Escisão \\
& & & & \\
& & & & \\
$\wp_{12}=0$ & $\hat{R}_{1.2345}^{2}=0,824102$ & & & \\
$\wp_{13}=0$ & $\hat{R}_{1.245}^{2}=0,507070$ & 11 & 11,34 & Rej $H_{0}$ \\
$\wp_{14}=0$ & $\hat{R}_{1.235}^{2}=0,606978$ & 11 & 8,84 & Rej $H_{0}$ \\
$\wp_{15}=0$ & $\hat{R}_{1.234}^{2}=0,639879$ & 11 & 7,88 & Rej $H_{0}$ \\
$\wp_{12}=\wp_{13}=0$ & $\hat{R}_{1.45}^{2}=0,259654$ & 10 & 14,37 & Rej $H_{0}$ \\
$\wp_{12}=\wp_{14}=0$ & $\hat{R}_{1.35}^{2}=0,079904$ & 10 & 16,54 & Rej $H_{0}$ \\
$\wp_{12}=\wp_{15}=0$ & $\hat{R}_{1.34}^{2}=0,270356$ & 10 & 14,22 & Rej $H_{0}$ \\
$\wp_{13}=\wp_{14}=0$ & $\hat{R}_{1.25}^{2}=0,426134$ & 10 & 11,80 & Rej $H_{0}$ \\
$\wp_{13}=\wp_{15}=0$ & $\hat{R}_{1.24}^{2}=0,486859$ & 10 & 10,71 & Rej $H_{0}$ \\
$\wp_{14}=\wp_{15}=0$ & $\hat{R}_{1.23}^{2}=0,548526$ & 10 & 9,42 & Rej $H_{0}$ \\
$\wp_{12}=\wp_{13}=\wp_{14}=0$ & $\hat{R}_{1.5}^{2}=0,012623$ & 9 & $15 ; 53$ & Rej $H_{0}$ \\
$\wp_{12}=\wp_{13}=\wp_{15}=0$ & $\hat{R}_{1.4}^{2}=0,196415$ & 9 & 13,67 & Rej $H_{0}$ \\
$\wp_{12}=\wp_{14}=\wp_{15}=0$ & $\hat{R}_{1.3}^{2}=0,030573$ & 9 & 15,36 & Rej $H_{0}$ \\
$\wp_{13}=\wp_{14}=\wp_{15}=0$ & $\hat{R}_{1.2}^{2}=0.423783$ & 9 & 10,68 & Rej $H_{0}$ \\
\hline
\end{tabular}


TABELA 12: Resultados dos Testes de Hipóteses para a equação (82)

\begin{tabular}{lllrl}
\hline Hipótese & $\hat{R}_{2 . l}^{2}$ & $I-s$ & $\begin{array}{r}W=(I-s) * \\
L n\left(\frac{1-\hat{R}_{2.1345}^{2}}{1-\hat{R}_{2.1}^{2}}\right)\end{array}$ & $\begin{array}{l}\text { Decisão } \\
\text { Estat. }\end{array}$ \\
\hline$\wp_{21}=0$ & $\hat{R}_{2.1345}^{2}=0,6905716$ & & & \\
$\wp_{23}=0$ & $\hat{R}_{2.345}^{2}=0,148213$ & 11 & 11,14 & Rej $H_{0}$ \\
$\wp_{24}=0$ & $\hat{R}_{2.145}^{2}=0,426192$ & 11 & 6,79 & Rej $H_{0}$ \\
$\wp_{25}=0$ & $\hat{R}_{2.135}^{2}=0,601111$ & 11 & 2,79 & Acei $H_{0}$ \\
$\wp_{21}=\wp_{23}=0$ & $\hat{R}_{2.134}^{2}=0,574112$ & 11 & 3,51 & Acei $H_{0}$ \\
$\wp_{21}=\wp_{24}=0$ & $\hat{R}_{2.45}^{2}=0,138180$ & 10 & 10,24 & Rej $H_{0}$ \\
$\wp_{21}=\wp_{25}=0$ & $\hat{R}_{2.35}^{2}=0,066169$ & 10 & 11,05 & Rej $H_{0}$ \\
$\wp_{23}=\wp_{24}=0$ & $\hat{R}_{2.34}^{2}=0,137104$ & 10 & 10,26 & Rej $H_{0}$ \\
$\wp_{23}=\wp_{25}=0$ & $\hat{R}_{2.15}^{2}=0,424432$ & 10 & 6,21 & Rej $H_{0}$ \\
$\wp_{24}=\wp_{25}=0$ & $\hat{R}_{2.14}^{2}=0,424629$ & 10 & 6,20 & Rej $H_{0}$ \\
$\wp_{21}=\wp_{23}=\wp_{24}=0$ & $\hat{R}_{2.13}^{2}=0 ; 564782$ & 10 & 3,41 & Acei $H_{0}$ \\
$\wp_{21}=\wp_{23}=\wp_{25}=0$ & $\hat{R}_{2.4}^{2}=0,098959$ & 9 & 10,46 & Rej $H_{0}$ \\
$\wp_{21}=\wp_{24}=\wp_{25}=0$ & $\hat{R}_{2.3}^{2}=0,065480$ & 9 & 9,62 & Rej $H_{0}$ \\
$\wp_{23}=\wp_{24}=\wp_{25}=0$ & $\hat{R}_{2.1}^{2}=0,423783$ & 9 & 9,95 & Rej $H_{0}$ \\
\hline & & & 5,60 & Acei $H_{0}$ \\
\hline
\end{tabular}


TABELA 13: Resultados dos Testes de Hipóteses para a equação (83)

\begin{tabular}{rllrl}
\hline Hipótese & $\hat{R}_{3 . l}^{2}$ & $I-s$ & $\begin{array}{r}W=(I-s) * \\
L n\left(\frac{1-\hat{R}_{3.1245}^{2}}{1-\hat{R}_{3.1}^{2}}\right)\end{array}$ & $\begin{array}{l}\text { Decisão } \\
\text { Estat. }\end{array}$ \\
\hline & & & & \\
$\wp_{31}=0$ & $\hat{R}_{3.1245}^{2}=0,772357$ & & & \\
$\wp_{32}=0$ & $\hat{R}_{3.145}^{2}=0,362065$ & 11 & 11,33 & Rej $H_{0}$ \\
$\wp_{34}=0$ & $\hat{R}_{3.125}^{2}=0,499919$ & 11 & 6,79 & Rej $H_{0}$ \\
$\wp_{35}=0$ & $\hat{R}_{3.124}^{2}=0,356399$ & 11 & 8,66 & Rej $H_{0}$ \\
$\wp_{31}=\wp_{32}=0$ & $\hat{R}_{3.45}^{2}=0,354551$ & 10 & 11,43 & Rej $H_{0}$ \\
$\wp_{31}=\wp_{34}=0$ & $\hat{R}_{3.25}^{2}=0,269920$ & 10 & 10,42 & Rej $H_{0}$ \\
$\wp_{31}=\wp_{35}=0$ & $\hat{R}_{3.24}^{2}=0,082927$ & 10 & 11,65 & Rej $H_{0}$ \\
$\wp_{32}=\wp_{34}=0$ & $\hat{R}_{3.15}^{2}=0,278525$ & 10 & 13,93 & Rej $H_{0}$ \\
$\wp_{32}=\wp_{35}=0$ & $\hat{R}_{3.14}^{2}=0,130503$ & 10 & 11,53 & Rej $H_{0}$ \\
$\wp_{34}=\wp_{35}=0$ & $\hat{R}_{3.12}^{2}=0,267799$ & 10 & 11,53 & Rej $H_{0}$ \\
$\wp_{31}=\wp_{32}=\wp_{34}=0$ & $\hat{R}_{3.5}^{2}=0,225767$ & 9 & 13,40 & Rej $H_{0}$ \\
$\wp_{31}=\wp_{32}=\wp_{35}=0$ & $\hat{R}_{3.4}^{2}=0,042388$ & 9 & 11,02 & Rej $H_{0}$ \\
$\wp_{31}=\wp_{34}=\wp_{35}=0$ & $\hat{R}_{3.2}^{2}=0,065480$ & 9 & 12,93 & Rej $H_{0}$ \\
$\wp_{32}=\wp_{34}=\wp_{35}=0$ & $\hat{R}_{3.1}^{2}=0,030573$ & 9 & 12,71 & Rej $H_{0}$ \\
\hline
\end{tabular}


TABELA 14: Resultados dos Testes de Hipóteses para a equação (84)

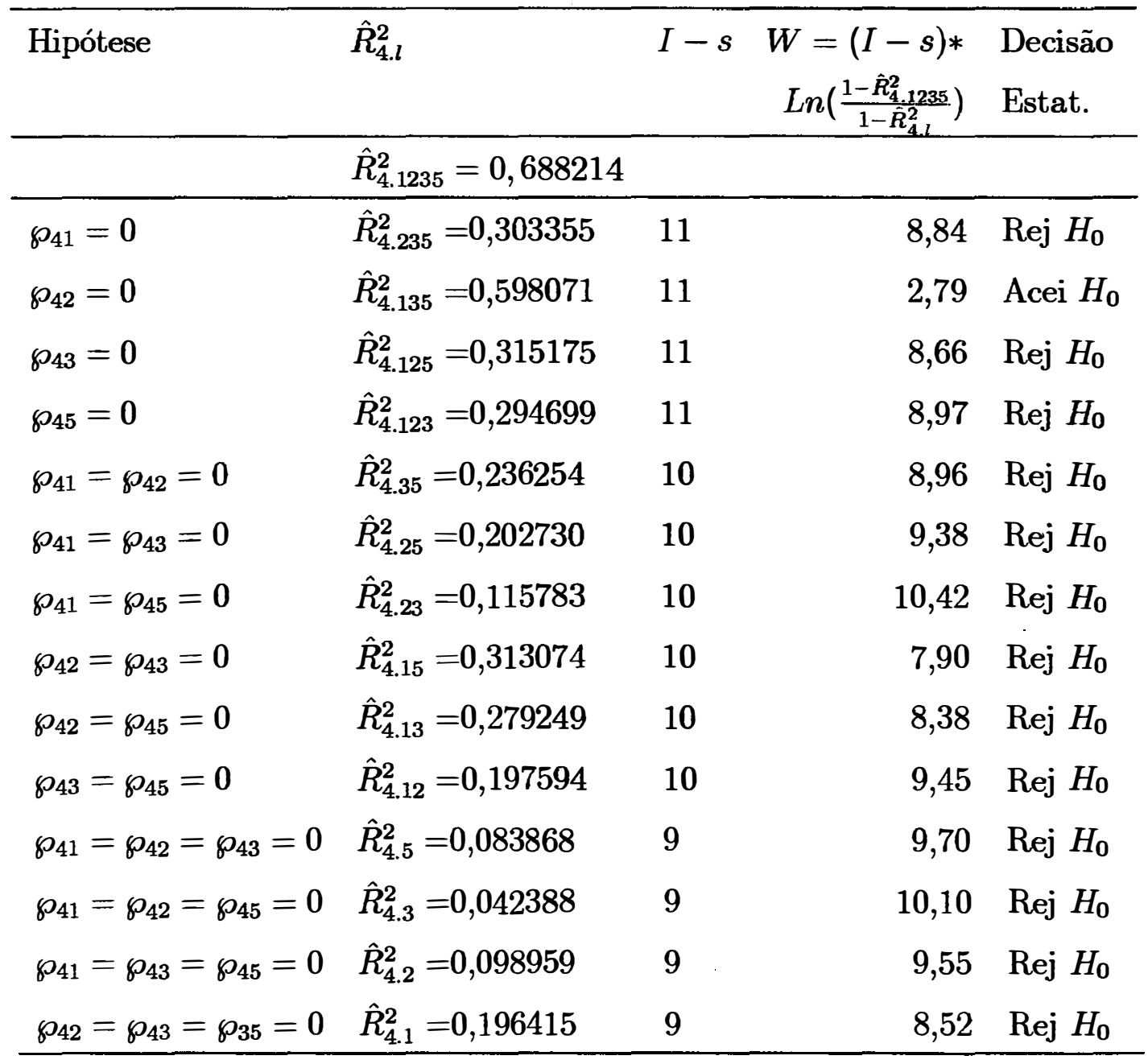


TABELA 15: Resultados dos Testes de Hipóteses para a equação ( 85)

\begin{tabular}{lllrl}
\hline Hipótese & $\hat{R}_{5 . l}^{2}$ & $I-s$ & $\begin{array}{r}W=(I-s) * \\
L n\left(\frac{1-\hat{R}_{5.1234}^{2}}{1-\hat{R}_{5.1}^{2}}\right)\end{array}$ & $\begin{array}{l}\text { Decisão } \\
\text { Estat. }\end{array}$ \\
\hline$\wp_{51}=0$ & $\hat{R}_{5.1234}^{2}=0,702273$ & & & \\
$\wp_{52}=0$ & $\hat{R}_{5.234}^{2}=0,390458$ & 11 & 7,88 & Rej $H_{0}$ \\
$\wp_{53}=0$ & $\hat{R}_{5.134}^{2}=0,590217$ & 11 & 3,51 & Acei $H_{0}$ \\
$\wp_{54}=0$ & $\hat{R}_{5.124}^{2}=0,158257$ & 11 & 11,43 & Rej $H_{0}$ \\
$\wp_{51}=\wp_{52}=0$ & $\hat{R}_{5.123}^{2}=0,326504$ & 11 & 8,98 & Rej $H_{0}$ \\
$\wp_{51}=\wp_{53}=0$ & $\hat{R}_{5.34}^{2}=0,382509$ & 10 & 7,29 & Rej $H_{0}$ \\
$\wp_{51}=\wp_{54}=0$ & $\hat{R}_{5.24}^{2}=0,123745$ & 10 & 10,79 & Rej $H_{0}$ \\
$\wp_{52}=\wp_{53}=0$ & $\hat{R}_{5.23}^{2}=0,226339$ & 10 & 9,55 & Rej $H_{0}$ \\
$\wp_{52}=\wp_{54}=0$ & $\hat{R}_{5.14}^{2}=0,155964$ & 10 & 10,42 & Rej $H_{0}$ \\
$\wp_{53}=\wp_{54}=0$ & $\hat{R}_{5.13}^{2}=0,2651656$ & 10 & 9,03 & Rej $H_{0}$ \\
$\wp_{51}=\wp_{52}=\wp_{53}=0$ & $\hat{R}_{5.12}^{2}=0,0137348$ & 10 & 11,97 & Rej $H_{0}$ \\
$\wp_{51}=\wp_{52}=\wp_{54}=0$ & $\hat{R}_{5.3}^{2}=0,225767$ & 9 & 10,12 & Rej $H_{0}$ \\
$\wp_{51}=\wp_{53}=\wp_{54}=0$ & $\hat{R}_{5.2}^{2}=0,009693$ & 9 & 8,60 & Rej $H_{0}$ \\
$\wp_{52}=\wp_{53}=\wp_{54}=0$ & $\hat{R}_{5.1}^{2}=0,0126228$ & 9 & 10,82 & Rej $H_{0}$ \\
\hline
\end{tabular}


FIGURA 8: Gráfico de trajetória final para as espécies Drosophyla

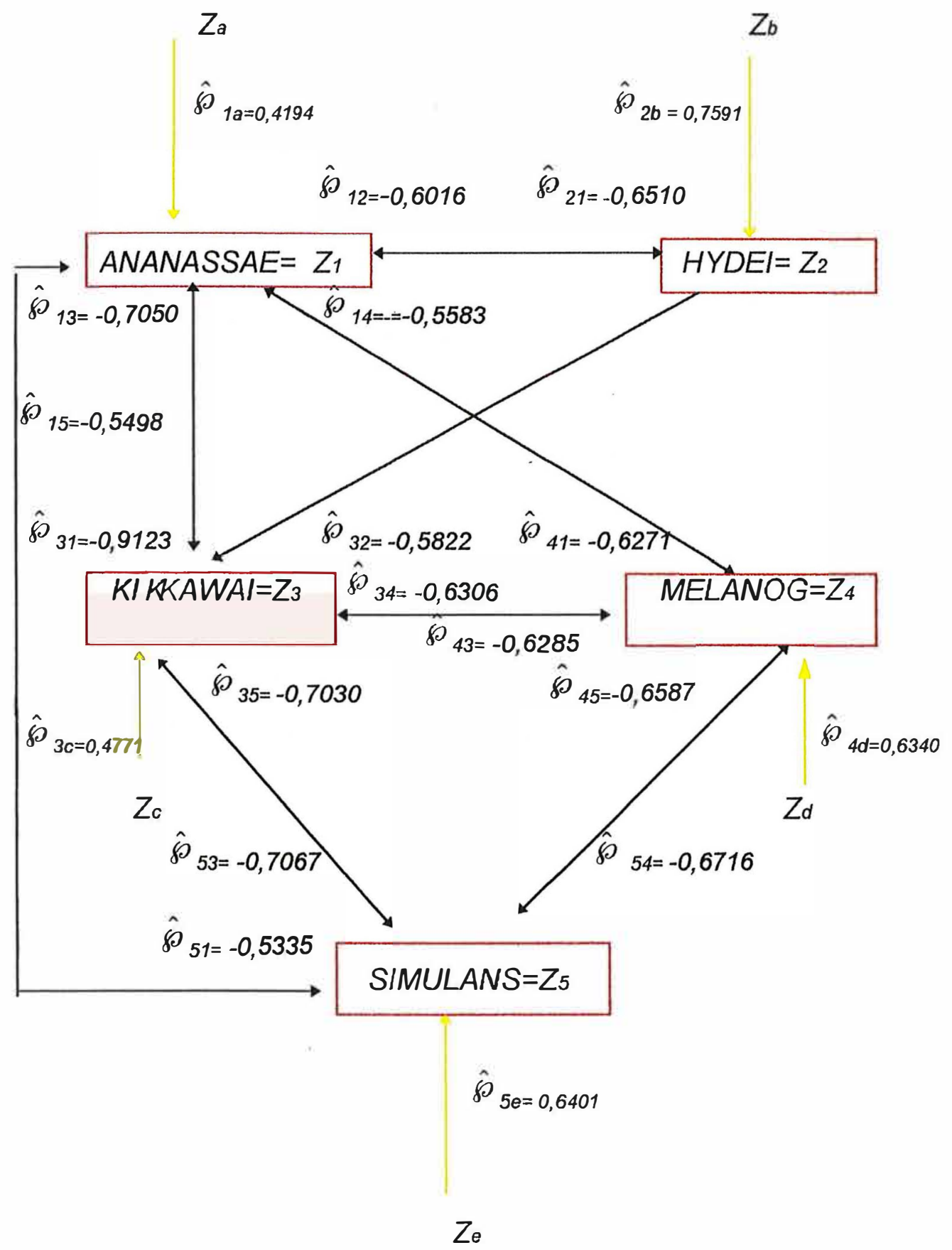


com efeito residual $\hat{\wp}_{5 e}=\left(1-\hat{R}_{5.134}^{2}\right)^{1 / 2}=0,6401$.

Logo, considerando-se os resultados dos testes de hipóteses, o gráfico de trajetórias final poderia ser o da Figura 8.

Observa-se, por exemplo, que a espécie HYDEI só tem o efeito direto da espécie ANANASSAE e com $\hat{\wp}_{21}=-0,6510$.

Levando em conta as trajetórias da Figura 8, mais uma vez pode-se usar o Teorema Fundamental para decompor os coeficientes de codependência.

Logo, a decomposição final dos coeficientes de co-dependência, associado ao Grafo da Figura 8 está na Tabela 16. 
TABELA 16: Decomposição Final dos Coeficientes de Co-dependência

\begin{tabular}{ccc}
\hline Co-dependências & \multicolumn{2}{c}{ Coeficientes de Associação } \\
& Diretos & Outros \\
\hline$\hat{\delta}_{12}=-0,6510$ & $\hat{\wp}_{12}=-0,6016$ & $-0,0494$ \\
$\hat{\delta}_{13}=-0,1748$ & $\hat{\wp}_{13}=-0,7049$ & $+0,5301$ \\
$\hat{\delta}_{14}=-0,4432$ & $\hat{\wp}_{14}=-0,5583$ & 0,1151 \\
$\hat{\delta}_{15}=-0,1124$ & $\hat{\wp}_{15}=-0,5498$ & $+0,4374$ \\
$\hat{\delta}_{21}=-\mathbf{0 , 6 5 1 0}$ & $\hat{\wp}_{21}=-\mathbf{0 , 6 5 1 0}$ & - \\
$\hat{\delta}_{31}=-0,1749$ & $\hat{\wp}_{31}=-0,9123$ & 0,7374 \\
$\hat{\delta}_{32}=-0,2559$ & $\hat{\wp}_{32}=-0,5822$ & 0,3263 \\
$\hat{\delta}_{34}=-0,2059$ & $\hat{\wp}_{34}=-0,6306$ & 0,4247 \\
$\hat{\delta}_{35}=-0,4752$ & $\hat{\wp}_{35}=-0,7030$ & 0,2278 \\
$\hat{\delta}_{41}=-0,4432$ & $\hat{\wp}_{41}=-\mathbf{0 , 6 2 7 1}$ & 0,1839 \\
$\hat{\delta}_{43}=-0,2059$ & $\hat{\wp}_{43}=-\mathbf{0 , 6 2 8 5}$ & 0,4226 \\
$\hat{\delta}_{45}=-0,2896$ & $\hat{\wp}_{45}=-\mathbf{0 , 6 5 8 7}$ & 0,3691 \\
$\hat{\delta}_{51}=-0,1124$ & $\hat{\wp}_{51}=-\mathbf{0 , 5 3 3 5}$ & 0,4211 \\
$\hat{\delta}_{53}=-0,4752$ & $\hat{\wp}_{53}=-\mathbf{0 , 7 0 6 7}$ & 0,2315 \\
$\hat{\delta}_{54}=-0,2896$ & $\hat{\wp}_{54}=-\mathbf{0 , 6 7 1 6}$ & 0,3820 \\
\hline
\end{tabular}

Também, foram realizadas as mesmas análises com os dados da Tabela 6 para os níveis de saúde mental.

Nas tabelas 17, 18, 19 e 20 apresentam-se: os coeficientes de codependências, as dependências e co-dependências, os coeficientes de trajetórias e os resultados dos testes de hipótese propostos.

Se os resultados dos testes de hipóteses propostos fossem válidos 
os níveis de saúde mental: Formação Sintomática Suave e Formação Sintomática Moderada poderiam ser eliminados do esquema de associação, segundo os resultados obtidos ao propor o teste: $H_{0}: \wp_{42}=\wp_{43}=0$. Observa-se que este resultado é compatível com o obtido pela eliminação de níveis de saúde mental através de análise de dependência (Cordeiro, 1990).

TABELA 17: Coeficientes de Co-dependência entre os níveis de Saúde Mental

\begin{tabular}{l|l|rrrr}
\hline Níveis & Boa & FS Suave & FS Moderada & Má \\
\hline Boa & 1 & 0,709787 & $-0,281123$ & $-0,956016$ \\
FS Suave & 0,709787 & 1 & $-0,652741$ & $-0,657068$ \\
FS Moderada & $-0,281123$ & $-0,652741$ & 1 & 0,069843 \\
Má & $-0,956016$ & $-0,657068$ & 0,069843 & 1 \\
\hline
\end{tabular}

TABELA 18: Dependências e Co-dependências entre os níveis de saúde mental

\begin{tabular}{l|l|rrrr}
\hline Níveis & Boa & FS Suave & FS Moderada & Má \\
\hline Boa & 0,001775 & 0,000276 & $-0,000138$ & $-0,001641$ \\
FS Suave & 0,000276 & 0,000085 & $-0,00007$ & $-0,000247$ \\
FS Moderada & $-0,000138$ & $-0,000070$ & 0,000136 & 0,000033 \\
Má & $-0,001641$ & $-0,000247$ & 0,000033 & 0,016601 \\
\hline
\end{tabular}


FIGURA 9: Gráfico de trajetória para Saúde Mental

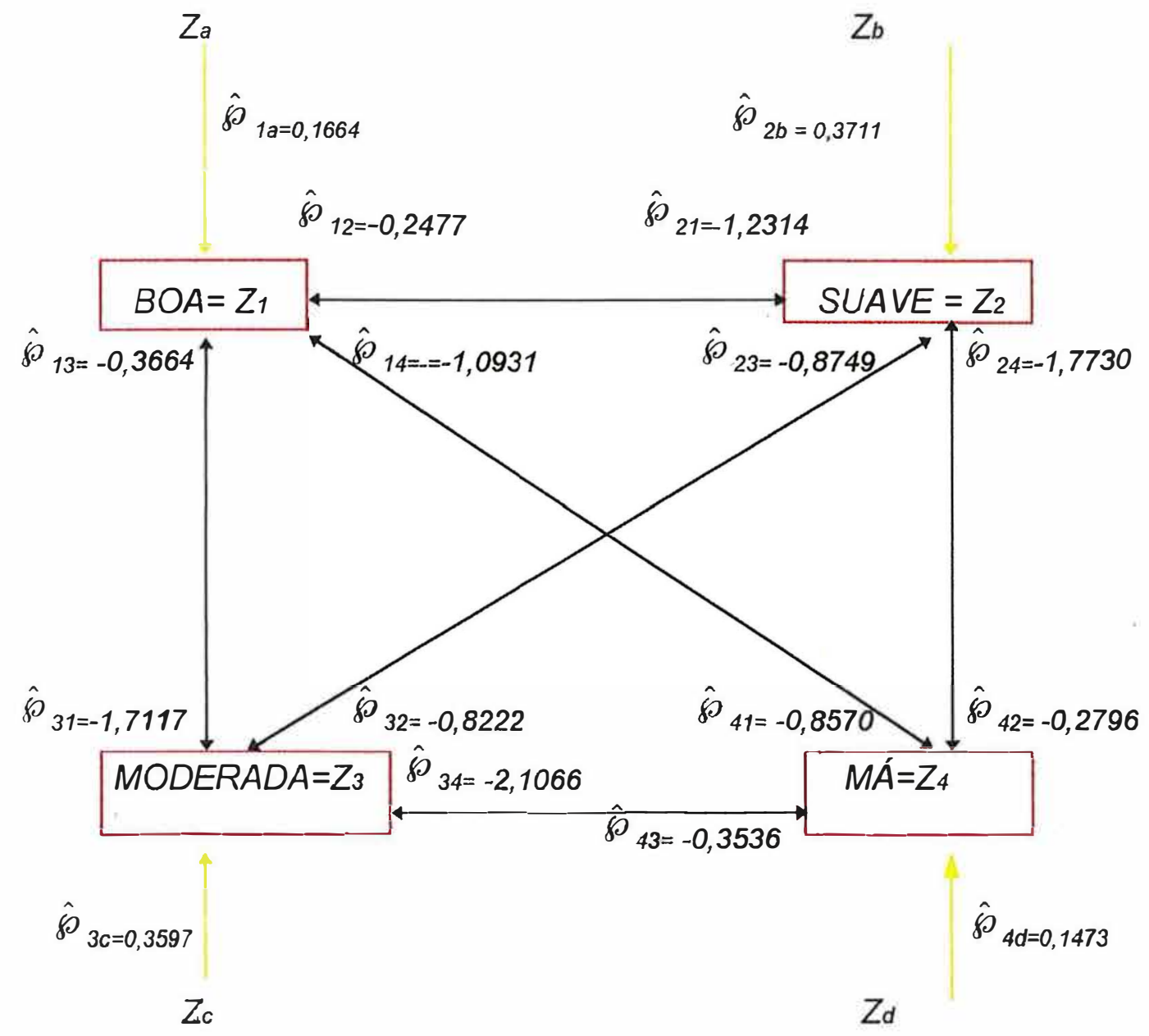


TABELA 19: Decomposição dos Coeficientes de Co-dependência

\begin{tabular}{cc}
\hline $\begin{array}{c}\text { Coeficientes } \\
\text { de Co-dependências }\end{array}$ & Coeficientes de Associação \\
\hline$\hat{\delta}_{12}=0,7098$ & $\hat{\wp}_{12}=-0,2477$ \\
$\hat{\delta}_{13}=-0,2811$ & $\hat{\wp}_{13}=-0,3664$ \\
$\hat{\delta}_{14}=-0,9560$ & $\hat{\wp}_{14}=-1,0931$ \\
$\hat{\delta}_{21}=0,7098$ & $\hat{\wp}_{21}=-1,2314$ \\
$\hat{\delta}_{23}=-0,6527$ & $\hat{\wp}_{23}=-0,8749$ \\
$\hat{\delta}_{24}=-0,6571$ & $\hat{\wp}_{24}=-1,7730$ \\
$\hat{\delta}_{31}=-0,2811$ & $\hat{\wp}_{31}=-1,7117$ \\
$\hat{\delta}_{32}=-0,6527$ & $\hat{\wp}_{35}=-0,8222$ \\
$\hat{\delta}_{34}=0,0698$ & $\hat{\wp}_{34}=-2,1066$ \\
$\hat{\delta}_{41}=-0,9560$ & $\hat{\wp}_{41}=-0,8570$ \\
$\hat{\delta}_{42}=-0,6571$ & $\hat{\wp}_{42}=-0,2796$ \\
$\hat{\delta}_{43}=0,0698$ & $\hat{\wp}_{43}=-0,3536$ \\
\hline
\end{tabular}


TABELA 20: Resultados dos Testes de Hipóteses para os dados de Saúde mental

\begin{tabular}{rlrrl}
\hline Hipótese & $\hat{R}_{1 . l}^{2}$ & $I-s$ & $\begin{array}{r}W=(I-s) * \\
\operatorname{Ln}\left(\frac{1-\hat{R}_{1,234}^{2}}{1-R_{1 . l}^{2}}\right)\end{array}$ & Decisão \\
& & & & \\
& $\hat{R}_{1.234}^{2}=0,9723$ & & & \\
$\wp_{12}=0$ & $\hat{R}_{1.34}^{2}=0,960138$ & 5 & 1,8199 & Acei $H_{0}$ \\
$\wp_{13}=0$ & $\hat{R}_{1.24}^{2}=0,925689$ & 5 & 4,9341 & Rej $H_{0}$ \\
$\wp_{14}=0$ & $\hat{R}_{1.23}^{2}=0,561629$ & 5 & 13,8081 & Rej $H_{0}$ \\
$\wp_{12}=\wp_{13}=0$ & $\hat{R}_{1.4}^{2}=0,91397$ & 4 & 4,5332 & Acei $H_{0}$ \\
$\wp_{12}=\wp_{14}=0$ & $\hat{R}_{1.3}^{2}=0,07903$ & 4 & 14,0159 & Rej $H_{0}$ \\
$\wp_{13}=\wp_{14}=0$ & $\hat{R}_{1.2}^{2}=0,50379$ & 4 & 11,5422 & Rej $H_{0}$ \\
Hipótese & $\hat{R}_{2 . l}^{2}$ & $I-s$ & $W=(I-s) *$ & Decisão \\
& & & $L n\left(\frac{1-\hat{R}_{2.134}^{2}}{1-R_{2}^{2}}\right)$ & Estat. \\
\hline$\wp_{21}=0$ & $\hat{R}_{2.34}^{2}=0,801809$ & 5 & 1,8199 & Acei $H_{0}$ \\
$\wp_{23}=0$ & $\hat{R}_{2.14}^{2}=0,509171$ & 5 & 6,3543 & Rej $H_{0}$ \\
$\wp_{24}=0$ & $\hat{R}_{2.13}^{2}=0,726816$ & 5 & 3,4245 & Acei $H_{0}$ \\
$\wp_{21}=\wp_{23}=0$ & $\hat{R}_{2.4}^{2}=0,431738$ & 4 & 5,6694 & Acei $H_{0}$ \\
$\wp_{24}=0$ & $\hat{R}_{2.3}^{2}=0,426071$ & 4 & 5,7091 & Acei $H_{0}$ \\
$\wp_{24}=0$ & $\hat{R}_{2.1}^{2}=0,503798$ & 4 & 5,1270 & Acei $H_{0}$ \\
\hline
\end{tabular}


TABELA 20: Resultados dos Testes de Hipóteses para os dados de Saúde mental

\begin{tabular}{|c|c|c|c|c|}
\hline Hipótese & $\hat{R}_{3 . l}^{2}$ & $I-s$ & $\begin{array}{r}W=(I-s) * \\
\operatorname{Ln}\left(\frac{1-\hat{R}_{3,124}^{2}}{1-\hat{R}_{3,}^{2}}\right)\end{array}$ & $\begin{array}{l}\text { Decisão } \\
\text { Estat. }\end{array}$ \\
\hline & \multicolumn{4}{|l|}{$\hat{R}_{3.124}^{2}=0,870629$} \\
\hline$\wp_{31}=0$ & $\hat{R}_{3.24}^{2}=0,65294$ & 5 & 4,93414 & $\operatorname{Rej} H_{0}$ \\
\hline$\wp_{32}=0$ & $\hat{R}_{3.14}^{2}=0,53893$ & 5 & 6,35433 & Rej $H_{0}$ \\
\hline$\wp_{34}=0$ & $\hat{R}_{3.12}^{2}=0,49296$ & 5 & 6,82950 & Rej $H_{0}$ \\
\hline$\wp_{31}=\wp_{32}=0$ & $\hat{R}_{3.4}^{2}=0,00488$ & 4 & 8,16072 & $\operatorname{Rej} H_{0}$ \\
\hline$\wp_{31}=\wp_{34}=0$ & $\hat{R}_{3.2}^{2}=0,42607$ & 4 & 5,95929 & Rej $H_{0}$ \\
\hline$\wp_{32}=\wp_{34}=0$ & $\hat{R}_{3.1}^{2}=0,07903$ & 4 & 7,85097 & Rej $H_{0}$ \\
\hline \multirow[t]{3}{*}{ Hipótese } & $\hat{R}_{4 . l}^{2}$ & $I-s$ & $W=(I-s) *$ & Decisão \\
\hline & & & $\operatorname{Ln}\left(\frac{1-\hat{R}_{4.123}^{2}}{1-\hat{R}_{4 \perp}^{2}}\right)$ & Estat. \\
\hline & \multicolumn{4}{|l|}{$\hat{R}_{4.123}^{2}=0,978286$} \\
\hline$\wp_{41}=0$ & $\hat{R}_{4.23}^{2}=0,65636$ & 5 & 13,8082 & Rej $H_{0}$ \\
\hline$\wp_{42}=0$ & $\hat{R}_{4.13}^{2}=0,956928$ & 5 & 3,4245 & Acei $H_{0}$ \\
\hline$\wp_{43}=0$ & $\hat{R}_{4.12}^{2}=0,9148975$ & 5 & 6,8295 & Rej $H_{0}$ \\
\hline$\wp_{41}=\wp_{42}=0$ & $\hat{R}_{4.3}^{2}=0,004878$ & 4 & 15,2996 & Rej $H_{0}$ \\
\hline$\wp_{41}=\wp_{43}=0$ & $\hat{R}_{4.2}^{2}=0,431738$ & 4 & 13,0585 & Rej $H_{0}$ \\
\hline$\wp_{42}=\wp_{43}=0$ & $\hat{R}_{4.1}^{2}=0,913965$ & 4 & 5,5071 & Acei $H_{0}$ \\
\hline
\end{tabular}




\section{CONCLUSÕES}

Os resultados obtidos permitem as seguintes conclusões:

Com a transposição da metodologia de análise de trajetória para dados categóricos, mostrou-se a equivalência entre os dois tipos de formulações de análise de trajetória para dados categóricos em tabelas bidimensionais, através de relações funcionais: funções de regressão e de funções de trajetória.

A partir das definições de coeficientes de co-dependência parciais, coeficientes de co-dependência múltipla para tabelas de contingência bidimensionais, foi possível emular definições para os coeficientes de trajetórias em tabelas de contingência bidimensionais.

É possível, também, ser feita a abordagem através das relações entre os coeficientes de trajetória e os coeficientes de co-dependência parciais, bem como os coeficientes de co-dependência múltipla.

Pode ser feita a decomposição dos coeficientes de co-dependência, em tabelas de contingência de dupla entrada, nos efeitos diretos ( ou coeficientes de trajetória) e outros efeitos.

Os exemplos de aplicação ilustram a metodologia e suscitam aprofundamento do aspecto interpretativo. 
O teste de hipótese para significância dos coeficientes de caminhos, necessita ser melhor estudado, pois parece ser conservativo.

A proposta metodológica de análise de trajetória para dados categóricos, permite a realização das mesmas análises efetuadas em análise de trajetória com variáveis aleatórias numéricas. 


\section{REFERÊNCIAS BIBLIOGRÁFICAS}

ACHCAR, J. Análise de trajetória. São Paulo, 1976. 68p. Dissertação (Mestrado)- Instituto de Matemática e Estatística, Universidade de São Paulo.

AGRESTI, A. Analysis of ordinal categorical data. New York: John Wiley, 1984. 287p.

ANDERSEN, E. B. The statistical analysis of categorical data. New York: Springer Verlag, 1991. 531p.

ANDERSON, T.W. An introduction to multivariate statistical analysis. 2.ed. New York: John Wiley, 1984. 675p.

BENICIO, M. H. Fatores de baixo peso ao nascer em recém nascidos vivos no Município de São Paulo. São Paulo, 1983. 137p. Dissertação (Doutorado)- Faculdade de Medicina, Universidade de São Paulo.

BELO, M. ; LAVACA, P. Associação entre drosophila e leveduras. I. Atração e produtividade. Naturalia, n. 7, p. 35-45, 1982.

BISHOP, Y; FIENBERG, S.; HOLLAND, P. Discrete multivariate analysis: theory and practice. Cambridge: Mit Press, 1975. 557p.

BLALOCK, H.M. Correlation and causality: the multivariate case. Review Sociological, n. 39, p. 1246-1251, 1961.

CORDEIro, J. A. Análise de dependência. São José de Rio Preto, 1990. 34p. Tese (Livre Docência)- IBILCE, Universidade do Estado de São Paulo.

COX, D. R. Causality: some statistical aspects. Journal of the Royal Statistical Society, Ser. A, v. 155, n. 2, p. 291-301, 1992. 
COX, D.; WERMUTH, N. Linear dependencies represented by chain graphs. Statistical Science, v. 8, n. 3, p. 204-218, 1993.

DILLON, W. R. ; GOLDSTEIN, M. Multivariate analysis: methods and applications. New York: John Willey and Sons, 1984. 587p.

DOBSON, D. Introduction to gereralized models. 2. ed. London: Chapman and Hall, 1990. 174p.

DUNCAN, O. D. Path analysis: sociological exemples. American Journal of Sociology, v. 72, n. 1, p. 1-16, 1966.

FIENBERG, S. E. The analysis of cross- classified categorical data. Cambridge: The Mit Press, 1980. 198p.

GLYMOUR, C. A review of recent work on the foundations of causal in ference. Carnegie Mellon University and University of Pittsburgh, não publicada, 1993.

GOODMAN, L. A. The analysis of multidimensional contingency tables when some variables are posterior to others: a modified path analysis ap proach. Biometrika, v. 60, n. 1, p. 179-192, 1973.

GOODMAN, L. A. The analysis of cross- classified data having ordered and or unordered categories: association models correlation models and asymetrics models for contingency table with and without missing entries. The Annals of Statistics, v. 13, p. 10-69, 1985.

GREENACRE, M. J. Theory and applications of correspondence analysis. London: Academic Press, 1984. 364p.

GRIZZLE, J. ; STARMER, F. ; KOCH, G. Analysis of categorical data by linear models. Biometrics, n. 25, p. 489-504, 1969.

HOLLAND P. Statistics and causal inference. Journal of the American Statistical Association, v. 25, n. 1, p. 121-132, 1986.

KENDALL, M. \& STUART, A. The advanced theory of statistics. 2.ed. London: Charles Griffin, 1967. 690p.

KIIVERI, M. \& SPEED, A. Structural analysis of multivariate data. San 
Francisco: Jossey Bass, 1982.

LAURITZEN, S. Mixed graphical association models. Scandinavian Journal of Statistics, v. 16, p. 273-306, 1989.

LAURITZEN, S.; WERMUTH, N. Graphical models for association between variables, some of which are qualitative and some quantitative. The Annals of Statistics, v. 17, n. 1, p. 31-57, 1989.

LEBART, L. ; MORINEAU, A. ; FÉNELON, J. Traitment des donnés statistiques. Paris: Dunod, 1982. 124p.

LI, C. Path analysis: a primer. Boxwood: Pacific Grover, 1975. 346p.

PRATT, J. ; SCHLAIFER, R. . An the interpretation and observation of laws. Journal of Econometrics, v. 39, p. 23-52, 1988.

RUBIN, D. Stimating causal effects of treatment in randomized and non- randomized studies. Journal of Educational Psychology, v. 66, p. 688-701, 1974.

RUBIN, D. Assignment to treatment group on the basic of a covariate. Journal of Educational Statistics, v. 2, n. 1, p.1-26, 1977.

RUBIN, D. Formal modes os statistical inference for causal efects. Journal of Statistical Planning and Inference, v. 25, p. 279-292, 1990.

SIMON, H. Spurious correlation. A causal interpretation. Journal of the Americam Statistical Association, v. 49, p. 467-479, 1954.

SUPPES, P. A probabilistic theory of causality. Amsterdam: Publishing Company. 1970. 131p.

TUKEY, J. Causation, regression and path analysis. In: KEMPTHORNE, O. et al Statistics and mathematics in biology. Iowa: Ames, 1954. eap. 3 p. $35-66$.

WERMUTH, N. Linear recursive equations, covariance selection, and path analysis. Journal of the Americam Statistical Association, v. 75, n. 372, p. 963-972, Dec. 1980.

WERMUTH, N.; LAURITZEN, S. Graphical and recursive models for contingency tables. Biometrika, v. 70, p. 537-552, 1983. 
WERMUTH, N.; LAURITZEN, S. On substantive research hypotheses, conditional independence graphs and graphical chain models. Journal of the Royal Statistical Society, Ser. B, v. 52, n. 1, p. 21-50, 1990.

WERMUTH N. Linear dependencies represented by chain graphs. Statistical Science v. 8, n. 3, p. 204-217, 1993.

WITTAKER, J. Graphical models and applied multivariate analysis. New York: John Willey and Sons, 1990. 245p.

WRIGHT, S. On the nature of size factors. Genetics, n. 3, p. 367-374, 1918.

WRIGHT, S. The method of path coefficients. The Annals Mathematics of Statistics, n. 5, p. 161-215, 1934.

WRIGHT, S. The interpretation of multivariate systems. In: KEMPTHORNE, O. Statistics and mathematics in biology. Local: editora, 1954. cap 2, p. 11-33.

WRIGHT, S. Path coefficients and path regression: Alternative or complemen tary concepts?. Biometrics, n. 16; p. 189-202, 1960.

YATES, R. The analysis of contingency tables with groupings based on quantitative characters. Biometrika v. 35, n. , p. 176-181, 1948. 